Review

\title{
Lipid Nanocarriers for Anti-HIV Therapeutics: A Focus on Physicochemical Properties and Biotechnological Advances
}

\author{
Maria J. Faria ${ }^{1, \dagger}$, Carla M. Lopes ${ }^{2, *,+} \mathbb{D}$, José das Neves ${ }^{3,4,5}$ (D) and Marlene Lúcio ${ }^{1,6, * \mathbb{D}}$ \\ 1 CF-UM-UP, Centro de Física das Universidades do Minho e Porto, Departamento de Física da Universidade \\ do Minho, 4710-057 Braga, Portugal; faria.mariajf@gmail.com \\ 2 FP-I3ID, FP-ENAS/CEBIMED, Fernando Pessoa Energy, Environment, and Health Research Unit/Biomedical \\ Research Center, Portugal and Faculty of Health Sciences, Fernando Pessoa University, \\ 4200-150 Porto, Portugal \\ 3 i3S, Instituto de Investigação e Inovação em Saúde, Universidade do Porto, 4200-135 Porto, Portugal; \\ j.dasneves@i3s.up.pt \\ 4 INEB, Instituto de Engenharia Biomédica, Universidade do Porto, 4200-135 Porto, Portugal \\ 5 CESPU, Instituto de Investigação e Formação Avançada em Ciências e Tecnologias da Saúde, \\ 4585-116 Gandra, Portugal \\ 6 CBMA, Centro de Biologia Molecular e Ambiental, Departamento de Biologia, Universidade do Minho, \\ 4710-057 Braga, Portugal \\ * Correspondence: cmlopes@ufp.edu.pt (C.M.L.); mlucio@fisica.uminho.pt (M.L.); \\ Tel.: +351-225-074-630 (C.M.L.) \\ + These authors contributed equally to this work.
}

check for

updates

Citation: Faria, M.J.; Lopes, C.M.; das Neves, J.; Lúcio, M. Lipid Nanocarriers for Anti-HIV

Therapeutics: A Focus on

Physicochemical Properties and Biotechnological Advances. Pharmaceutics 2021, 13, 1294. https://doi.org/10.3390/ pharmaceutics13081294

Academic Editor: Nejat Düzgüneş

Received: 19 July 2021

Accepted: 7 August 2021

Published: 19 August 2021

Publisher's Note: MDPI stays neutral with regard to jurisdictional claims in published maps and institutional affiliations.

Copyright: (c) 2021 by the authors. Licensee MDPI, Basel, Switzerland. This article is an open access article distributed under the terms and conditions of the Creative Commons Attribution (CC BY) license (https:// creativecommons.org/licenses/by/ $4.0 /)$.

\begin{abstract}
Since HIV was first identified, and in a relatively short period of time, AIDS has become one of the most devastating infectious diseases of the 21st century. Classical antiretroviral therapies were a major step forward in disease treatment options, significantly improving the survival rates of HIV-infected individuals. Even though these therapies have greatly improved HIV clinical outcomes, antiretrovirals (ARV) feature biopharmaceutic and pharmacokinetic problems such as poor aqueous solubility, short half-life, and poor penetration into HIV reservoir sites, which contribute to the suboptimal efficacy of these regimens. To overcome some of these issues, novel nanotechnology-based strategies for ARV delivery towards HIV viral reservoirs have been proposed. The current review is focused on the benefits of using lipid-based nanocarriers for tuning the physicochemical properties of ARV to overcome biological barriers upon administration. Furthermore, a correlation between these properties and the potential therapeutic outcomes has been established. Biotechnological advancements using lipid nanocarriers for RNA interference (RNAi) delivery for the treatment of HIV infections were also discussed.
\end{abstract}

Keywords: ARV delivery; biotechnology in ARV; biological barriers; lipid emulsions; lipid nanoparticles; liposomes; RNAi and ARV codelivery

\section{Introduction}

The human immunodeficiency virus (HIV) is known to promote the continuous deterioration of the host immune system, being responsible for the acquired immunodeficiency syndrome (AIDS) [1,2]. According to the Joint United Nations Program on HIV infection/AIDS (UNAIDS), by the end of 2018, the epidemic accounted for more than 30 million deaths worldwide with a particular incidence in the female population and Sub-Saharan African countries [3]. Currently, 37.9 million people are infected with the virus and only a fraction $(\approx 82 \%)$ have access to antiretroviral (ARV) therapy [3].

ARVs revolutionized HIV infection/AIDS clinical history and their approval for therapeutic purposes transformed this condition into a chronically manageable disease [4]. ARV-based therapies continue to be the best treatment option against HIV infection/AIDS providing prolonged viral suppression and, consequently, lower mortality rates $[5,6]$. The 
first treatments were based on monotherapy regimens which rapidly led to the development of ARV resistance [7,8]. Consequently, novel strategies were adopted, namely, combined antiretroviral therapy (cART; formerly referred to as highly effective antiretroviral therapy (HAART)) based on the simultaneous administration of three or more different classes of drugs $[7,8]$.

Although HIV infection/AIDS stands as a public health concern, anti-HIV therapies have greatly increased the life quality and expectancy of infected individuals [9]. However, these therapies are challenging and often difficult to implement in the developing world. Multiple factors may compromise their success, such as: (i) adverse effects associated with the multi-regimen therapies extended over long periods; (ii) development of viral resistance; (iii) ineffective viral suppression due to low drug concentrations in viral reservoirs; (iv) pharmacokinetic problems and possible interactions between drugs; (v) poor stability and reduced shelf-life; (vi) low patient adherence; (vii) unbearable high costs for most of the populations in need, and (viii) socio-cultural constraints that limit the access to these treatments $[10,11]$.

In the last decades, several strategies to improve HIV disease management using nanotechnology have emerged and seen tremendous growth both in treatment and prevention. Nanotechnology-based systems radically changed the global medical scheme and gained considerable attention in therapeutic research. In particular, nanomedicine-based approaches may help to improve pharmacokinetic problems (e.g., low oral bioavailability or short half-life) of ARV drugs [9,12-21]. Poor aqueous solubility is another common problem transversal to many drugs, which can be improved by encapsulation in drug carriers [22]. The reduction of the particles size to the nanometric scale increases the surface area available for solvation which has shown to be an effective strategy to increase drug solubility and, consequently, improve oral bioavailability [23]. Another interesting feature brought by nanomedicine is the ability to modulate the drug release profiles to occur over a longer time and at higher effective doses to the specific sites $[8,22]$. Moreover, toxicity associated with ARV therapies may also be circumvented using drug-loaded systems. A possible explanation is the controlled release profiles obtained with nanocarriers, reducing the toxicity namely at the cellular level [2].

The encapsulation of ARV drugs is particularly interesting as a targeting strategy towards cellular and anatomic HIV reservoirs and it can be achieved either by passive or active targeting $[2,8]$. In the first case, the targeting is dependent on nanocarriers' intrinsic properties such as mean diameter, surface properties (e.g., charge), and shape [2]. On the other hand, active targeting typically depends on the functionalization of the carrier surface with ligands that recognize receptors at the targeted tissues [2,9]. Furthermore, these carriers act as protective shields against external threats (e.g., chemical and enzymatic degradation) leading to increasing residence periods of ARV in the organism [9]. This feature may promote the reduction of required doses and, consequently, prolong time intervals between administrations [2]. Ultimately, it is possible to encapsulate different types of therapeutic payloads within the same system which can contribute to simpler administrations increasing patient adherence but also reduce possible errors related to therapeutic regimens [2,24].

Among the multiple nanocarriers that can be used for ARV delivery, lipid-based nanocarriers hold great promise since 15 of the 21 marketed approved nanomedicines are liposomes or lipid nanoparticles (AmBisome ${ }^{\circledR}$, DaunoXome $^{\circledR}$, DepoCyt $^{\circledR}$, DepoDur $^{\circledR}$, Doxil $^{\circledR}$, Inflexal ${ }^{\circledR}$ V, Marqibo ${ }^{\circledR}$, Mepact $^{\circledR}$, , Myocet $^{\circledR}$, Visudyne ${ }^{\circledR}$, Abelcet ${ }^{\circledR}$, Amphotec $^{\circledR}$, Fungizone ${ }^{\circledR}$, Diprivan $^{\circledR}$, Estrasorb $^{\circledR}$ ) [25]. Of particular notice, this list has been recently upgraded with the introduction in the market of nucleoside-based nanomedicines for the treatment of hereditary transthyretin-mediated amyloidosis $\left(\right.$ Onpattro $\left.{ }^{\circledR}\right)$ and prophylaxis of severe acute respiratory syndrome coronavirus 2 (SARS-CoV-2) infection (Pfizer/BioNTech Comirnaty ${ }^{\circledR}$ and the Moderna COVID-19 vaccines). Furthermore, these carriers are well-accepted in the scientific community for therapeutic purposes mainly because their structural units are generally recognized as safe (GRAS) [25]. Additionally, lipids' biocompatibility and 
biodegradability properties as well as their versatility make them suitable and safe delivery systems for humans, with low or non-associated toxicity [11,26]. A large number of lipid-based nanocarriers developed for ARV delivery justifies a constantly updated review. Even though some reviews have covered this topic [27-29], it is important to address some neglected aspects regarding the details of formulation development to serve as a guide for researchers working in this field. To the best of our knowledge, no similar reviews have considered the composition and characterization of lipid nanocarriers in terms of size, colloidal stability, encapsulation, and drug loading efficiency, as well as establishing a correlation between the nanocarriers' physicochemical properties and their potential anti-HIV therapeutic outcomes. Moreover, biotechnological applications of lipid nanocarriers loaded with anti-HIV therapeutics will be presented including the use of lipoplexes for small interference ribonucleic acid (siRNA) delivery and other interesting prospects for other disease conditions (e.g., neurodegenerative diseases) that have not yet been considered. For example, considering that reverse transcriptase (RT) is found in a variety of human cells, including those in the brain, and that it is involved in somatic gene recombination (SGR), which is linked to dysregulated neuronal genomes in Alzheimer's disease (AD), the inhibition of this enzyme by ARV agents in combination with siRNAmediated silencing of its expression is considering a promising biotechnological approach for the prevention and/or treatment of this neurodegenerative disease. The utilization of lipid-based nanocarriers for co-delivery ARV and siRNA aids to cross the brain-blood barrier (BBB).

\section{ARV Agents: Mechanism of Action and Limitations}

Following the isolation and subsequent identification of HIV as the main agent responsible for the onset of HIV infection/AIDS, significant progress was made, allowing for a detailed characterization of the virus and its life cycle, as well as a better understanding of the mechanisms underlying its mode of action [30]. In this way, it became possible to identify new, highly specific pharmacological targets in the HIV life cycle (Figure 1) that allowed the development of the first drugs that would change the course of HIV infection/AIDS history.

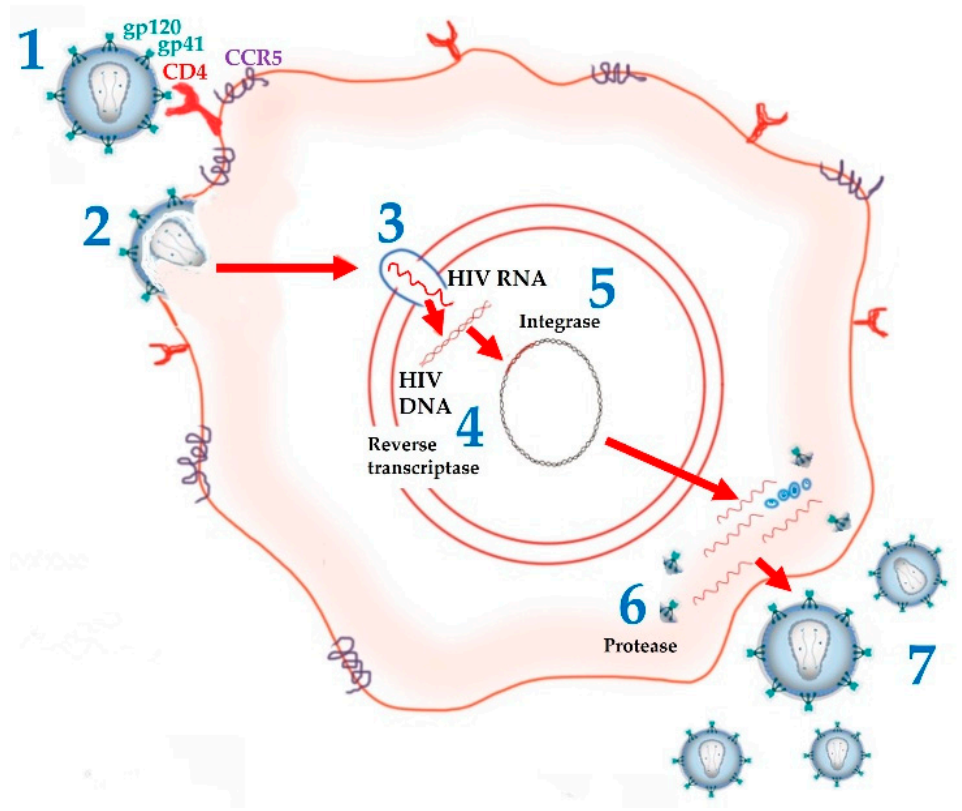

Figure 1. Stages in HIV lifecycle. (1) HIV attaches to CD4 receptor and CCR5 co-receptor. (2) HIV gp41 is exposed to the host cell and causes fusion. (3) HIV enters the nucleus and releases its enzymes and RNA. (4) Reverse transcriptase makes a double strand HIV DNA from HIV RNA. (5) Integrase includes HIV DNA in the DNA of the host cell. (6) New HIV viral components are produced, and Protease assembles new HIV virus. (7) Each host cell produces hundreds of new virions. 
In 1987, zidovudine (AZT) was approved as the first ARV for therapeutic use. Since then, and in a short period, 6 more classes of ARVs have been developed and, to date, 49 medicines containing single ARV drugs or drug associations (as in the case of cART) have been approved and made available on the market by the United States Food and Drug Administration (U.S. FDA) for the clinical treatment of HIV infection/AIDS [31]. The existing ARV drugs can be classified according to their target [4] (Figures 2-6).

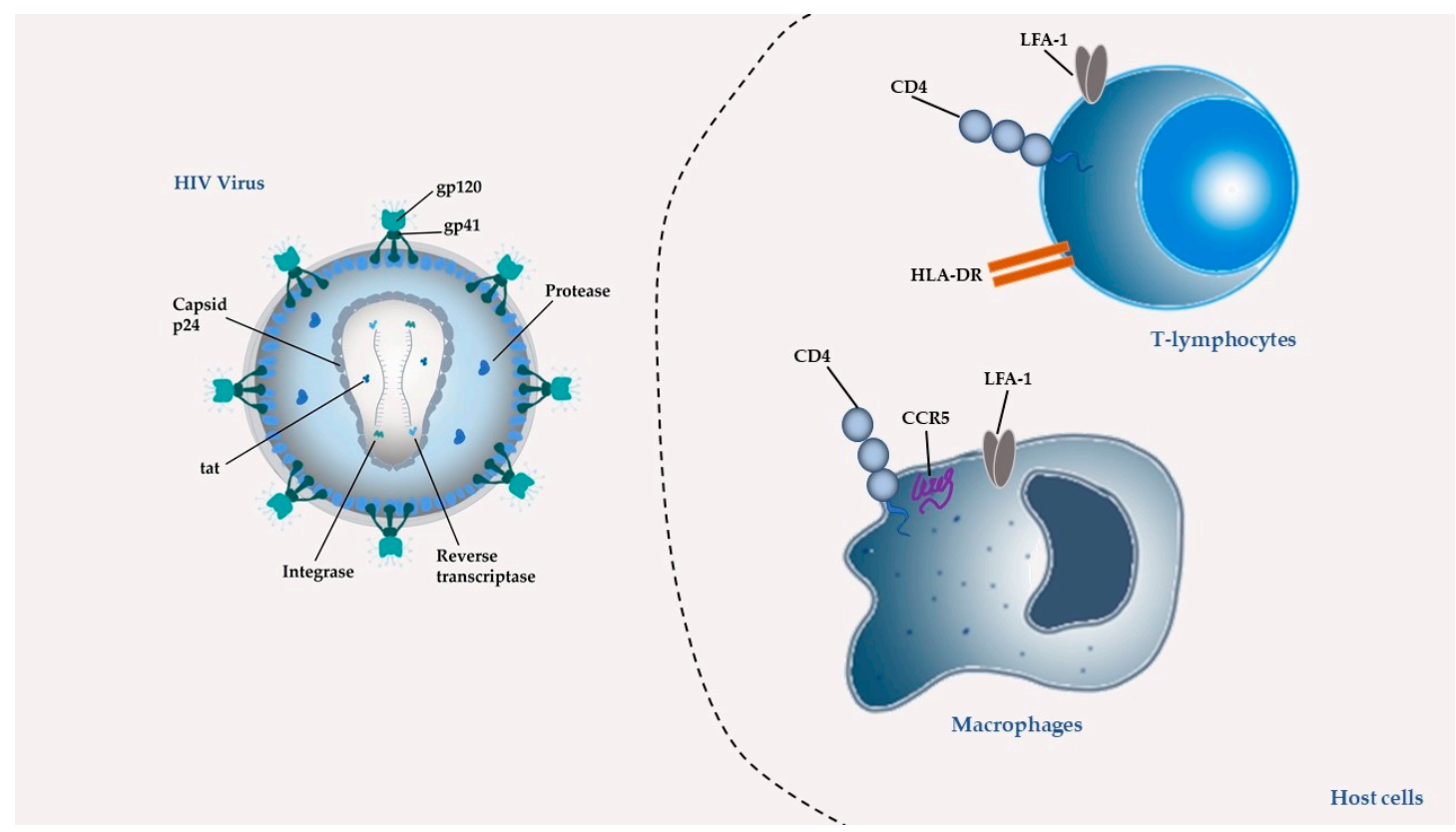

Figure 2. Molecular components of HIV virus and targets of ARV drugs. In the virus, the targets can be the glycoproteins responsible for adhesion gp120 and gp41; the enzymes integrase, reverse transcriptase, and protease; the protein from the capsid p24 and protein Tat that modulates transcription initiation and can reactivate a latently infected cell by penetrating. In the host cells the targets can be the lymphocyte function-associated antigen 1 (LFA-1); the CD4 receptor and its co-receptor C-C Motif Chemokine Receptor 5 (CCR5); and the human leucocyte antigen (HLA-DR).

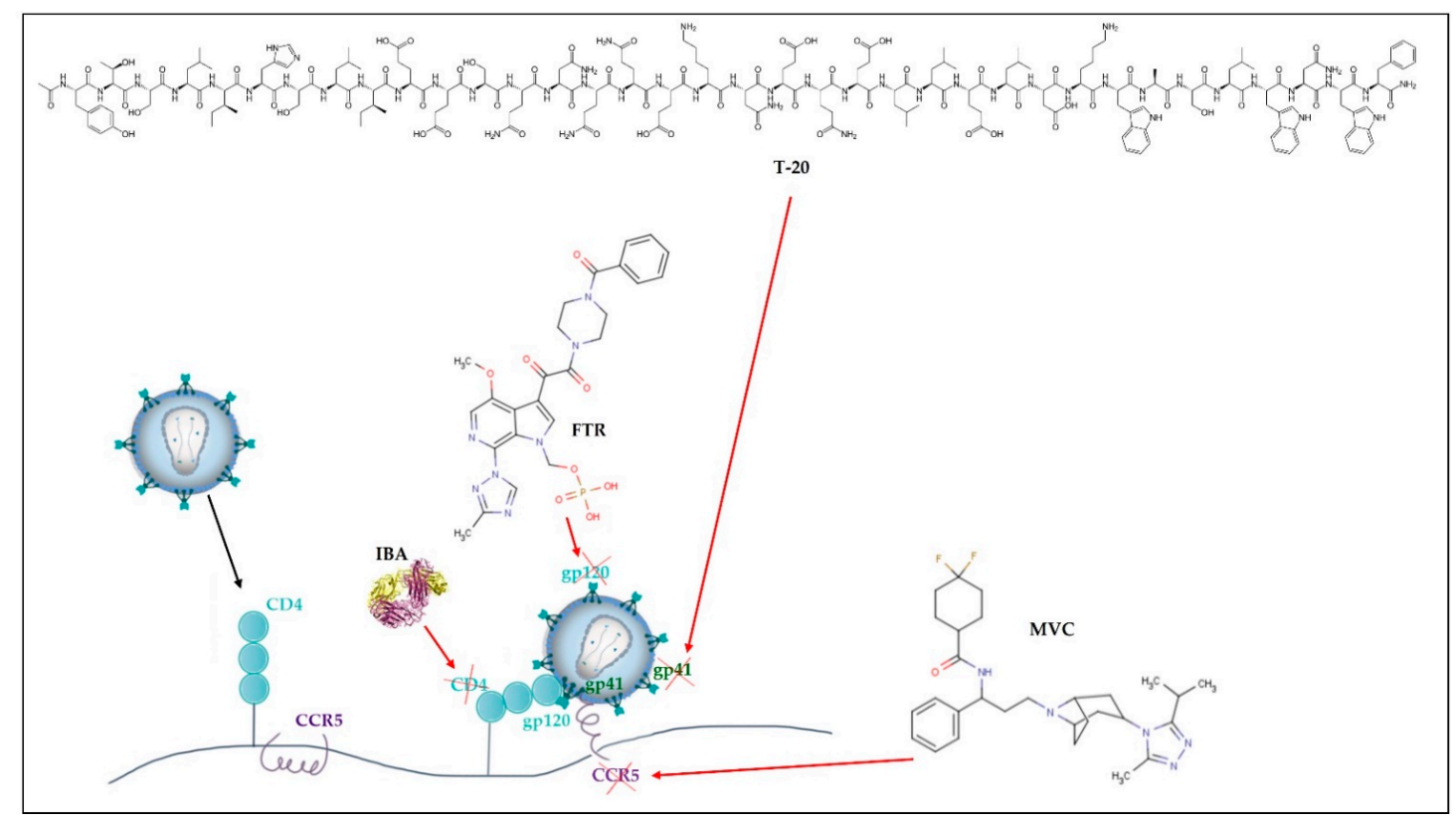

Figure 3. Cell entry inhibitors and fusion inhibitors. Ibalizumab-uiyk (IBA) blocks CD4 and maraviroc (MVC), blocks CCR5 receptors from host cells. Enfuvirtide (T-20) blocks gp41 and fostemsavir tromethamine (FTR) blocks gp120 from the virus. 


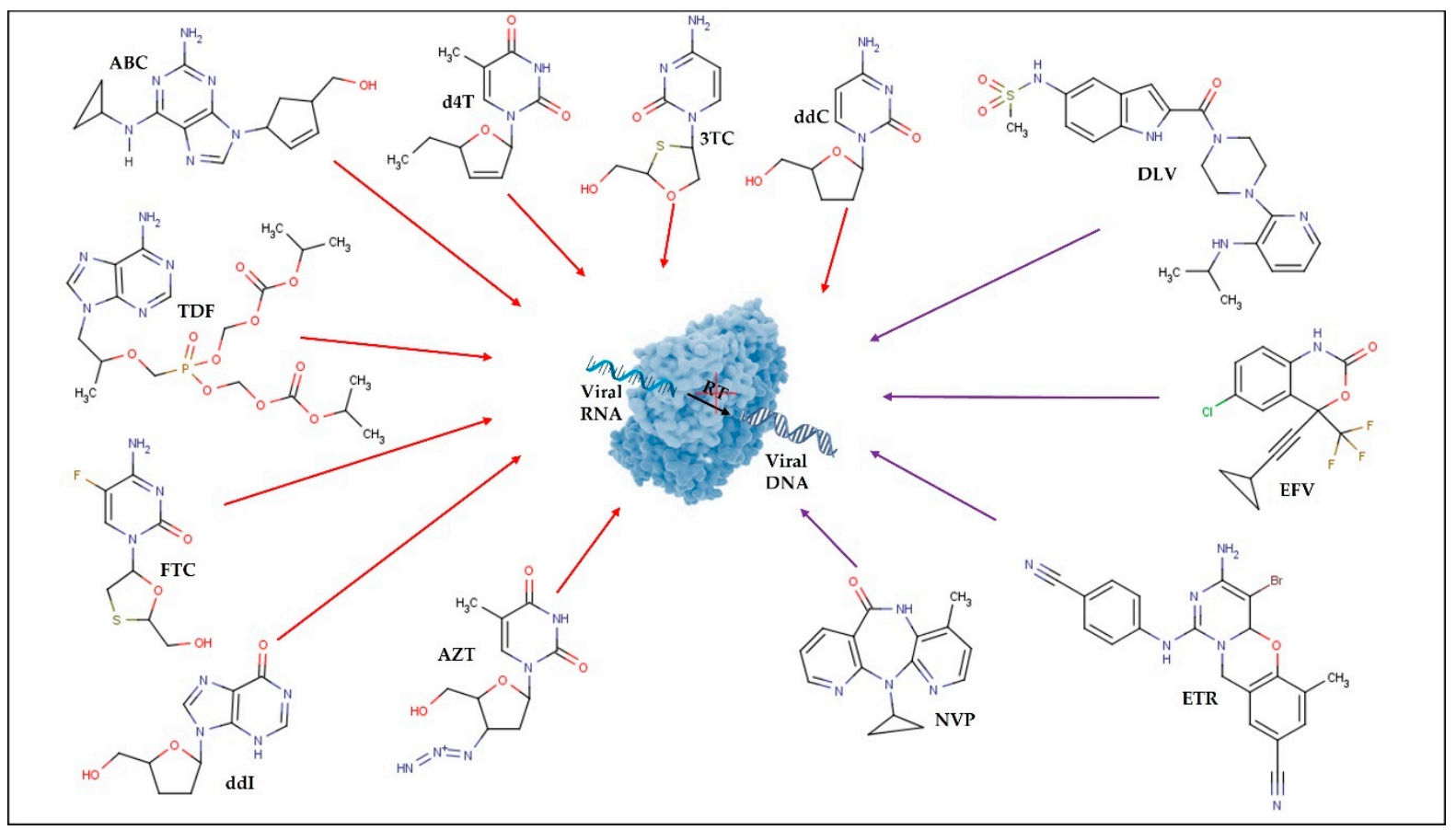

Figure 4. Reverse transcriptase (RT) inhibitors. Red arrows represent the nucleoside reverse transcriptase inhibitors (NRTI): lamivudine (3TC); abacavir (ABC); zidovudine (AZT); stavudine (d4T); didanosine (ddI); zalcitabine (ddC); emtricitabine (FTC); tenofovir disoproxil fumarate (TDF). Purple arrows represent the non-nucleoside reverse transcriptase inhibitors (NNRTI): efavirenz (EFV); etravirine (ETR); nevirapine (NVP); delavirdine (DLV).

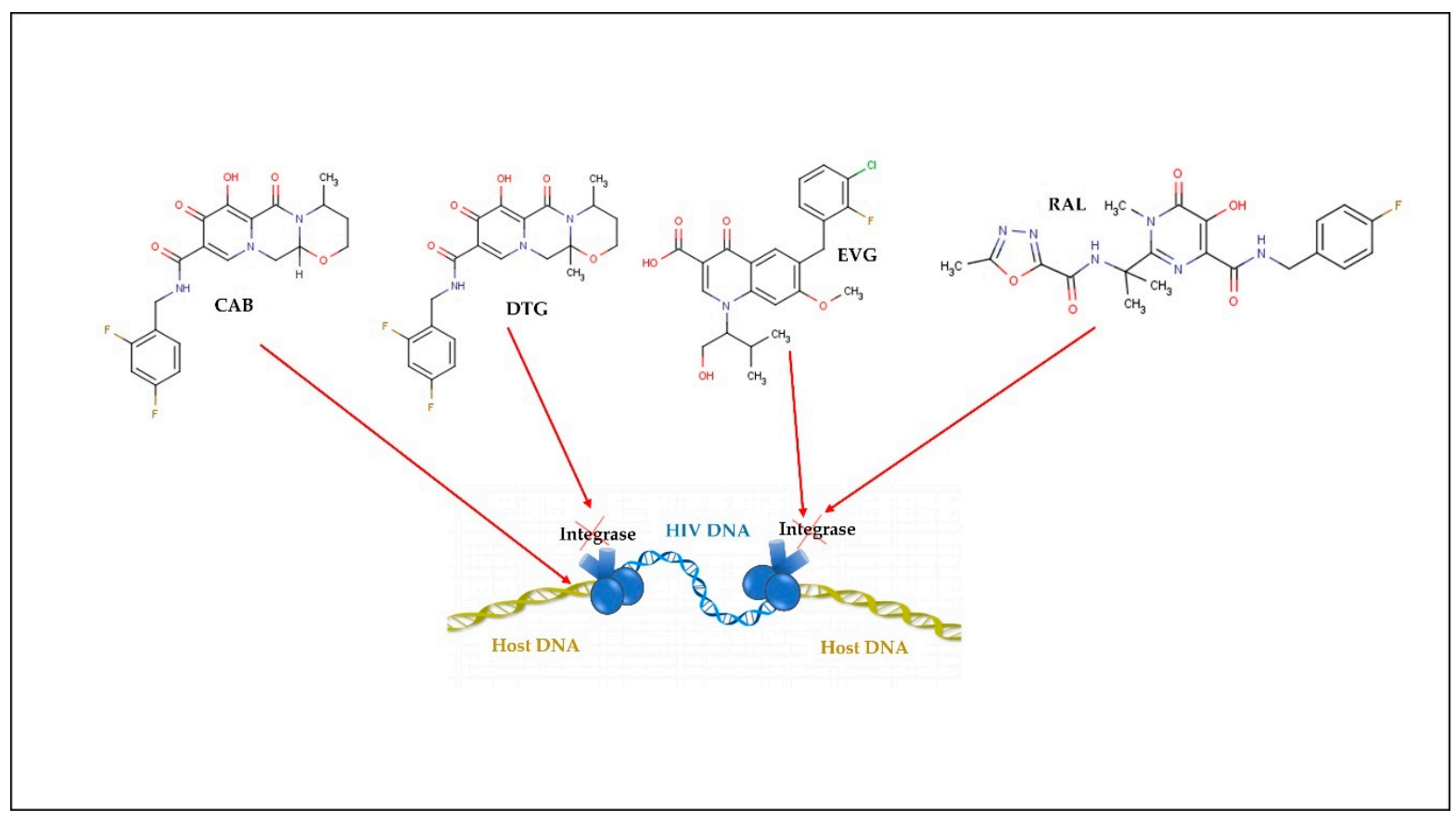

Figure 5. Integrase inhibitors: raltegravir (RAL); dolutegravir (DTG); elvitegravir (EVG); cabotegravir (CAB). 


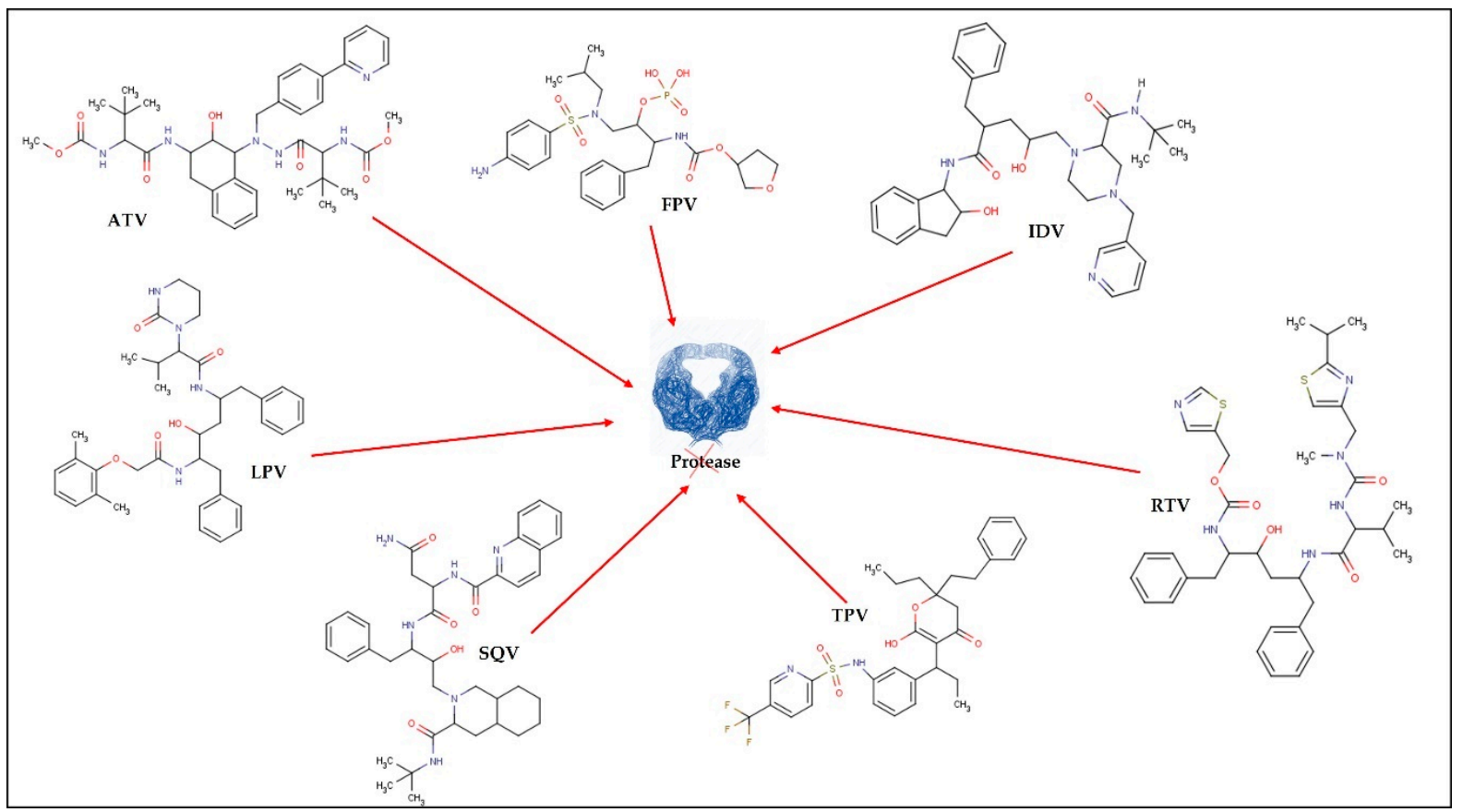

Figure 6. Protease inhibitors: tipranavir (TPV); indinavir (IDV); ritonavir (RTV); fosamprenavir (FPV); atazanavir (ATV); lopinavir (LPV); saquinavir (SQV).

Thus, ARV can be grouped into the following therapeutic classes (Table 1): cell entry inhibitors (stage 1 of Figures 1 and 3; fusion inhibitors (FI) (stage 2 of Figures 1 and 3; nucleoside reverse transcriptase inhibitors (NRTI), non-nucleoside RT inhibitors (NNRTI) (stage 4 of Figures 1 and 4); integrase inhibitors (IIs) (stage 5 of Figures 1 and 5); and protease inhibitors (PI) (stage 6 of Figures 1 and 6) [31,32]. Pharmacokinetic enhancing drugs (e.g., cobicistat) can also be used in association with ART agents to improve therapeutic effectiveness.

Table 1. ARV agents are classified into therapeutic classes based on their mechanism of action and target site [31,33,34].

ARV Therapeutic Class

CCR5 antagonists

Cell entry inhibitors

\section{Mechanism of Action}

Block CCR5 coreceptors present on the surface of specific immune cells, preventing HIV from entering the cells.

Bind to the gp120 protein on the viral outer surface, blocking HIV entry into CD4 cells.

Block CD4 receptors present on the surface of specific immune cells, preventing HIV from entering the cells.

Interferes with HIV binding, fusion, and cell entrance by preventing the

Fusion inhibitors (FI) gp41 glycoprotein from being

exposed to the virus-host cell membrane.$$
\text { membrane. }
$$

ARV Single Agents and Some ARV Associations

MVC

FTR

IBA 
Table 1. Cont.

\begin{tabular}{|c|c|c|}
\hline ARV Therapeutic Class & Mechanism of Action & $\begin{array}{l}\text { ARV Single Agents and } \\
\text { Some ARV Associations }\end{array}$ \\
\hline Nucleoside reverse transcriptase inhibitors (NRTI) & $\begin{array}{l}\text { Block the viral RT, inhibiting HIV } \\
\text { replication. }\end{array}$ & $\begin{array}{l}\text { 3TC; ABC; AZT; d4T; ddI; ddI } \\
\text { EC; ddC (F.M.); FTC; TDF; } \\
\text { 3TC+AZT; ABC+3TC; } \\
\text { ABC+AZT+3TC; TDF+FTC }\end{array}$ \\
\hline Non-nucleoside reverse transcriptase inhibitors (NNRTI) & $\begin{array}{l}\text { Bind to viral RT and subsequently } \\
\text { modify it, limiting HIV replication. }\end{array}$ & $\begin{array}{c}\text { DOR; EFV; RPV; ETR; NVP; } \\
\text { DLV }\end{array}$ \\
\hline Integrase inhibitors (II) & $\begin{array}{l}\text { Inhibition of viral integrase. } \\
\text { Prevents the incorporation of HIV } \\
\text { proviral DNA strands into the host } \\
\text { cell genome. }\end{array}$ & RAL; DTG; EVG; CAB \\
\hline Protease inhibitors (PI) & $\begin{array}{l}\text { Inhibition of viral protease. } \\
\text { Prevents the cleavage of some viral } \\
\text { proteins and the maturation of } \\
\text { virions, resulting in non-viral } \\
\text { particles. }\end{array}$ & $\begin{array}{c}\text { TPV; IDV; RTV; DRV; FPV; } \\
\text { ATV; LPV+RTV; SQVM+RTV }\end{array}$ \\
\hline
\end{tabular}

Abbreviations: 3TC, lamivudine; ABC, abacavir; ATV, atazanavir; AZT, zidovudine; CAB, cabotegravir; CCR5, C-C chemokine receptor type 5; CD4, cluster of differentiation 4; d4T, stavudine; ddC, zalcitabine; ddI, didanosine; ddI EC, enteric coated didanosine; DLV, delavirdine; DNA, deoxyribonucleic acid; DOR, doravirine; DRV, darunavir; DTG, dolutegravir; EFV, efavirenz; ETR, etravirine; EVG, elvitegravir; FI, fusion inhibitors; FPV, fosamprenavir; FTC, emtricitabine; FTR, fostemsavir tromethamine; gp41, glycoprotein gp41; gp120, glycoprotein gp120; HIV, human immunodeficiency virus; IBA, ibalizumab-uiyk; IDV, indinavir; II, integrase inhibitors; LPV, lopinavir; MVC, maraviroc; NNRTI, non-nucleoside reverse transcriptase inhibitors (NRTI, nucleoside reverse transcriptase inhibitors; NVP, nevirapine; PI, protease inhibitors; RAL, raltegravir; RT, reverse transcriptase; RPV, rilpivirine; RTV, ritonavir; SQVM, saquinavir mesylate; T-20, enfuvirtide; TDF, tenofovir disoproxil fumarate; TPV, tipranavir.

Despite the recognized overall success of cART, particularly in developing countries, this therapeutic strategy continues to raise some important issues, and its effectiveness is affected by several limitations. For example, the multi-dose treatments administered over extended periods can result in the development of ARV resistance mechanisms and can also lead to the inability to effectively suppress the virus due to the difficulties in maintaining consistent drug levels, particularly in viral reservoirs $[8,35]$. The mechanisms underlying ARV resistance are often related to HIV genetic variability and HIV reverse transcriptase processing errors. This high mutation rate coupled with the virus's fast replication leads to the creation of innumerable virus variants (quasispecies) capable of avoiding the immune system [36,37]. Additionally, the recombination of more than one viral strain during infection or the accumulation of proviral variants also contribute to viral resistance [37,38]. Although some HIV variants display primary mutations that make them less susceptible to ARV action, most ARV resistance results from direct exposure to these regimens and it was already observed in all six therapeutic classes through different mechanisms [36]. For example, in NRTI, whose main function is to block the viral RT and inhibit HIV replication, resistance can occur by two mechanisms. The first mechanism corresponds to mutations at or near the drug-binding site of RT (e.g., M184V, L74V, K65R, and others) leading to a conformational change in the enzyme that ultimately blocks the binding of NRTI to the active site $[36,39]$. Such a mechanism enables viral RT to discriminate between dideoxyNRTI chain terminators and endogenous triphosphate deoxynucleosides, preventing the binding of NRTI to viral DNA [37] The second mechanism corresponds to phosphorylytic removal of NRTI-triphosphate from its site of attachment in the viral DNA chain [36,37]. These mutations are characteristic of the thymidine analogs (AZT and d4T) and can also be described as thymidine analog mutations (TAM). TAM can be further divided into type I (e.g., M41L, L210W, and T215Y) and II (e.g., D67N, K70R, T215F, and K219Q/E), however, type I is responsible for higher levels of phenotypic and clinical cross-resistance [37].

The most frequent mutations that occur in NNRTIs, take place within their binding pocket and mostly affect hydrophobic residues of HIV-RT [36,40]. These mutations (e.g., L100I, G190S/A, and Y181C) alter the binding site of RT to NNRTIs which consequently 
decreases the binding affinity and alters the overall structure of the complex [39]. Other mutations (e.g., K103N) can act using a different mechanism such as the establishment of a hydrogen bond at the entrance of the binding pocket. This helps to maintain the pocket closed and limits NNRTI diffusion. Nevertheless, one of the biggest hurdles related to the use of NNRTI is that the binding site in RT is approximately the same for all of them, which means that a single mutation can lead to high-level drug resistance and cause cross-resistance among all NNRTI [36,41].

Furthermore, the resistance to PI is associated with mutations in the protease gene and subsequent replacement of amino acids within the protease enzyme (e.g., D30N, V32I, G48V, V82A, and others) [36,41]. These modifications will reduce the binding affinity between the catalytic binding site of the enzyme and the PI [36]. Other mutations in the enzyme flap (e.g., I54M/L) and core (e.g., L76V and N88S) can also decrease PI susceptibility [40]. In response to these mutations, the geometry of the catalytic site of the homodimer is enlarged disabling the inhibitor to effectively bind to the gene and block cleavage [36].

The development of resistance in FI is related to mutations in gp41 codons 36-45 (e.g., G36DEV, V38EA, Q40H, N42T, and N43D), correspondent to the location where T-20 will bind $[36,38]$. In the case of II, the occurrence of mutations (e.g., N155H, Q148R, Y143R, and others) at Asp64 and Asp116 carboxylate residues (which coordinate an $\mathrm{Mg}^{2+}$ ion) compromise the catalytic activity of the enzyme [41]. It is thought that the functional group of IIs binds selectively to the enzyme complexes which further interferes with strand transfer of viral and host DNA [41]. Finally, cell entry inhibitors such as maraviroc (CCR5 inhibitor) may develop resistance via gp120 mutations, enabling HIV to bind the CCR5-CCR5 inhibitor complex [40]. However, the most common mechanism of resistance to CCR5 inhibitors is associated with an enhancement of CXCR4 tropic viruses that are intrinsically insensitive to CCR5 inhibitors [40].

Besides the resistance mechanisms, prolonged treatment regimens often result in poor adherence and careless patient intake, as well as treatments with high associated costs $[2,42,43]$. In addition to this, any interruption in the therapeutic regimen results in treatment failure and viral resistance. Moreover, drug associations can improve the therapeutic effectiveness, but they may also have the opposite effect due to an increase in deleterious drug-drug interactions. Prolonged use of ARV therapeutic regimens is also often associated with toxic side effects (e.g., constipation/diarrhea, abdominal pain, nausea, liver and metabolic disorders, kidney stones, anemia, fatigue, headache, fever, muscular dystrophy, and peripheral neuropathy) that compromise the quality of life of patients [44].

Pharmacokinetic issues are another significant limitation of cART and single ARV therapies. In either case, ARV demonstrates low and unpredictable bioavailability after oral administration due to their poor gastrointestinal (GI) absorption, extensive first-pass metabolism, and GI enzymatic degradation. The majority of ARV drugs are classified in biopharmaceutical class system (BCS) II, III, or IV, which means they have low solubility and permeability. For drugs given orally, solubility is required to confirm drug absorption and clinical response. The speed and extent of oral drug diffusion through the mucus layer, submucosa, and epithelial cell barriers into the blood or lymphatic circulation is referred to as permeability. Low solubility and permeability thereby show that ARV drugs are poorly absorbed in the body [45]. Even after absorption, most ARV present other significant pharmacokinetic limitations, such as a short half-life that demands recurrent dose administration in a fastidious dosage regimen, which contributes to poor patient adherence $[2,44,45]$. Another pharmacokinetic issue is poor body distribution of ARV, which prevents reaching certain target tissues that serve as viral reservoirs. High plasma protein binding of ARV, for example, impairs drug permeation across the BBB [46]. The central nervous system (CNS) is known as an anatomical viral reservoir, where HIV survives in long-lived cells, such as microglia. As a result, viral eradication by ARV drugs or cART becomes more difficult and newer and drug-resistant HIV strains develop. Furthermore, some ARV drugs' inability to enter the CNS further restricts eradication. ARV drugs may also be expelled from CNS at the BBB level by efflux transporters like 
glycoprotein P (P-gp) [47]. Simultaneously, the inflammatory response induced by HIV infection of the CNS causes permeability increase of the BBB and plays an important role in neuropathogenesis $[47,48]$.

Moreover, ARV fails to target lymphatic system cells (e.g., dendritic cells and macrophages) involved in virus transmission to helper T lymphocytes (CD4+ T cells), resulting in posttreatment infection relapse [49].

To address the limitations of cART and single ARV therapies, there is an urge to develop innovative strategies, such as nanocarriers for ARV delivery. Among the vast types of nanocarriers available, lipid-based nanocarriers can be one of the most attractive drug carrier classes for ARVs. The ability of these systems to transport drugs of varying lipophilicity, as well as their widely accepted biocompatibility and biodegradability, make them appealing for translation into clinical settings [50]. Additionally, as the oral route is the preferred method of administration, lipid nanocarriers stimulate the secretion of endogenous biliary lipids enhancing the GI absorptive capacity of the carried ARV agents [51]. Consequently, bioavailability enhancement and better distribution over the cellular and organ target viral sites are expected. Indeed, lipid nanocarriers can protect ARV agents (single or on association) through their body path, reducing accumulation in non-target tissues (reducing toxic side effects) and improving doses at viral reservoirs, as well as, avoiding unwanted drug interactions between the multiple carried drugs. The more specific and controlled delivery of ARV agents provided by lipid nanocarriers may also enhance therapeutic efficiency by decreasing the need for frequent administration regimens, which ultimately increases patient adherence. There is also the need to seek out novel alternatives capable of overcoming the physiological barriers inherent to oral ARV drug administration. Therefore, lipid nanocarriers are likewise advantageous to explore different non-invasive routes like skin transdermal, intranasal, and topical vaginal administration (for pre-exposure prophylaxis purposes) [19,52-55].

Nanocarriers for ARV drugs delivery in the CNS have also proved useful in circumventing the BBB because of their potential to enhance drug permeability. Nanocarriers have a variety of properties that help them penetrate the BBB and deliver drugs to the CNS, such as a high surface-to-volume ratio, a positive surface charge (to take advantage of adsorptive mediated transport through the BBB), and a small and regulated size (less than $200 \mathrm{~nm}$ ) [47,56]. The charge and hydrophobicity of the nanocarriers' surface impact plasmatic protein adsorption, and therefore their absorption and/or rate of transcytosis. Nanocarriers coated with specific surface stabilizers may be useful in achieving greater drug levels in the brain when it comes to CNS administration. Polysorbate 80 is a nonionic surfactant that has been shown to improve brain delivery in a variety of nanocarriers by adsorbing different apolipoproteins once in the circulation, imitating lipoproteins in their receptor-mediated transcytosis pathway into the CNS [47].

Given all of the mentioned advantages of nanocarriers for ARV delivery, the following section will provide a more detailed view of the types of lipid nanocarriers and their engineering properties to improve ARV therapy.

\section{Lipid-Based Nanocarriers for Delivery of ARV Agents}

Lipid-based nanocarriers are organic nanosystems that self-organize upon input of energy into a supramolecular structure with the hydrophilic portions (anionic, cationic, or zwitterionic) exposed to the surrounding aqueous solvent and the hydrophobic portions (usually hydrocarbon chains) facing each other to reduce contact with aqueous solvent [50]. Self-assembly is a common manufacturing method of lipid-based nanocarriers that is spontaneous but driven by an input of energy and the hydrophobic effect [50]. Lipid-based nanocarriers' definitions and main characteristics are presented in Table 2. 
Table 2. Lipid nanocarriers description, schematic representation, and main advantages and disadvantages for ARV delivery.

\begin{tabular}{|c|c|c|c|}
\hline Type of Lipid Nanocarriers & Description and Main Characteristics & Advantages/Disadvantages for ARV Delivery & References \\
\hline ETHOSOMES & $\begin{array}{l}\text { - } \quad \text { Aqueous phases in the core and } \\
\text { surroundings of synthetic vesicles formed by } \\
\text { self-assembly of lipid bilayers. } \\
\text { Unilamellar (1 bilayer), oligolamellar (2-4 } \\
\text { bilayers), and multilamellar ( }>4 \text { bilayers) } \\
\text { classifications are based on the number of } \\
\text { lipid bilayers. } \\
\text { Small (100 nm), large (100-500 nm), and } \\
\text { giant (>500nm) are the classifications based } \\
\text { on their size. } \\
\text { Ethosomes are phospholipid-based vesicles } \\
\text { with high ethanol content (20-45\%). }\end{array}$ & $\begin{array}{l}\text { - } \quad \text { Biocompatible and biodegradable. } \\
\text { - } \quad \text { intraninistration routes are limited (mainly } \\
\text { - } \quad \text { Liposomes in their natural state are quickly } \\
\text { absorbed by the reticuloendothelial system and } \\
\text { cleared from circulation. This property has been } \\
\text { used to deliver ARVs to macrophages. } \\
\text { The transdermal delivery of ARV is achieved by } \\
\text { the incorporation of edge activators (e.g., } \\
\text { surfactants, monoolein forming transferosomes) } \\
\text { or ethanol (forming ethosomes) in the lipid } \\
\text { bilayer. } \\
\text { The protection of sensitive therapeutics can be } \\
\text { achieved by using antioxidant agents in their } \\
\text { composition (e.g., } \alpha \text {-tocopherol, forming } \\
\text { tocosome). } \\
\text { Can encapsulate hydrophilic, hydrophobic, or } \\
\text { amphiphilic drugs. } \\
\text { Limited hydrophilic drug-loading capacity. } \\
\text { Low long-term physical and biological stability, } \\
\text { which hinders their use for long-term drug } \\
\text { delivery. }\end{array}$ & {$[19,53,57,58]$} \\
\hline
\end{tabular}


Table 2. Cont.

\section{Type of Lipid Nanocarriers}

CUBOSOMES

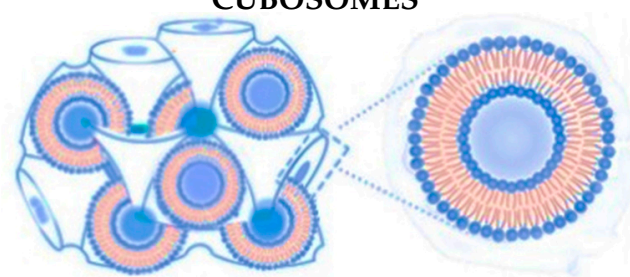

\section{Description and Main Characteristics}

- Highly stable structures organized in curved bicontinuous lipid bilayers forming soft 3D honeycomb-like structures.

- Composed by a continuous periodic bilayer and two non-connected water channels.

- $\quad$ Main components: glyceryl

monooleate/monoolein (GMO) and phytantriol.

\section{Advantages/Disadvantages for ARV Delivery}

- Incapability to modulate inner pore and channel sizes.

- Difficult loading of large molecules and difficult scale-up processes.

- $\quad$ Biocompatible and bioadhesive.

- Increase drug solubility and bioavailability through a variety of routes, including intranasal delivery to the brain and transdermal delivery.

- More stable than liposomes.

- High degree of encapsulation efficiency.

\section{LIPID NANOPARTICLES}

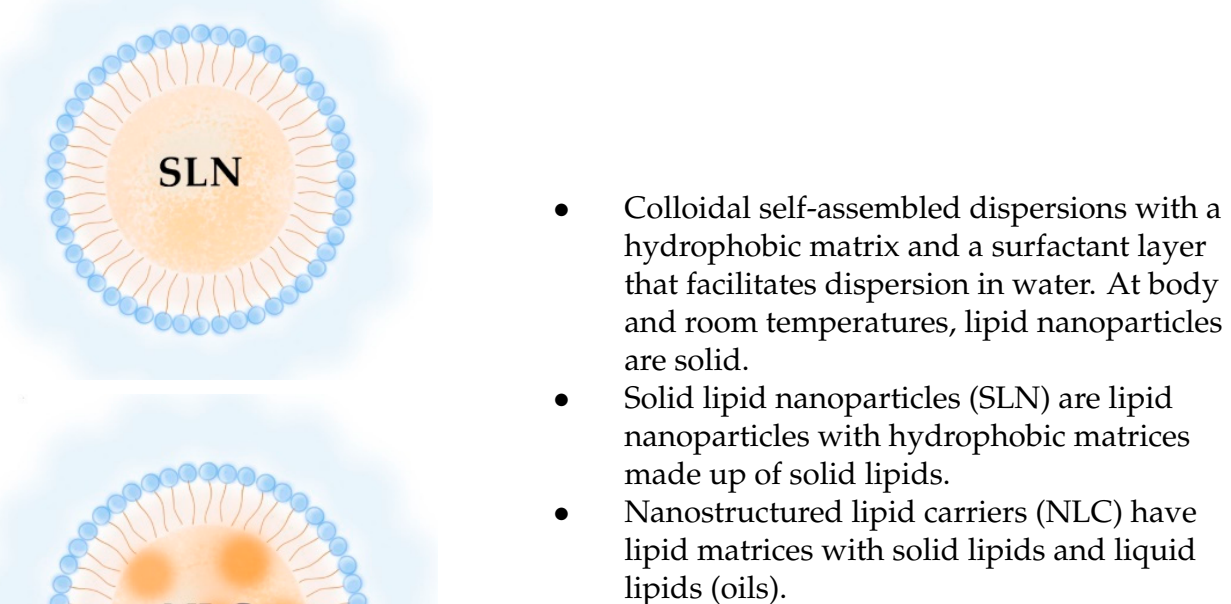

- $\quad$ Ease manufacturing and scale-up

- $\quad$ Low-cost and recognized as safe (GRAS) excipients, and biocompatibility.

- Greater drug stability and better control over drug-release kinetics than liposomes, cubosomes, and nanoemulsions.

- In comparison to other nanocarriers, they can entrap a greater amount of lipophilic drugs, but are inadequate for encapsulating hydrophilic and amphiphilic drugs. lipids (oils)

- Receptor-mediated transcytosis allows lipid nanoparticles to cross the BBB (targeting low-density lipoproteins receptors). 
Table 2. Cont.

\section{Type of Lipid Nanocarriers}

LIPID EMULSIONS

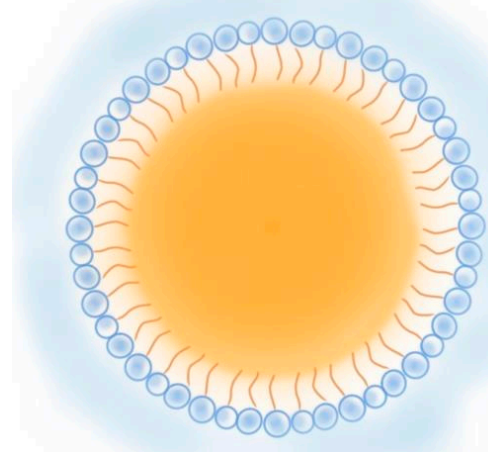

Nanoemulsion O/W

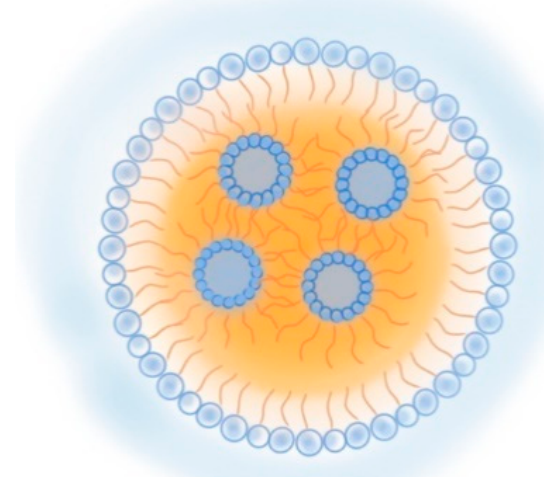

Description and Main Characteristics

- Colloidal systems made of immiscible liquid phases, categorized in water-in-oil (w/o) or oil-in-water $(\mathrm{o} / \mathrm{w})$, according to the phase dispersed in the other phase (continuous phase) and stabilized by surfactants.

- Microemulsions are thermo-dynamically stable dispersions that can be generated with low external energy. The droplet sizes of the dispersed phase are $<1000 \mathrm{~nm}$, typically range between 10 and $200 \mathrm{~nm}$, resulting in optically clear dispersion.

- Nanoemulsions are thermo-dynamically unstable and require high external energy to be produced. The dispersed phase droplets are $<500 \mathrm{~nm}$ typically $100 \mathrm{~nm}$. Over time, nanoemulsions are more prone to instability.

- $\quad$ Self-emulsifying drug delivery systems (SEDDS) are emulsions that, when gently agitated, form fine oil-in-water droplets without the need for a dissolution process. These include self-micro emulsifying delivery systems (SMEDDS) with droplet sizes < $50 \mathrm{~nm}$; self-nanoemulsifying drug delivery systems (SNEDDS) with droplet sizes of 20 to $200 \mathrm{~nm}$; and solid self-nanoemulsifying oily formulations (S-SNEOF) where the drug is precipitated as a result of the evaporation of the co-solvent.
Advantages/Disadvantages for ARV Delivery

References

- Increase drug oral bioavailability as their droplets preserve the drug from gastrointestinal degradation and can be dispersed quickly in

blood and lymph (thereby avoiding the first-pass metabolism), but are also administrated by other routes: topical, and intravenous.

- $\quad$ Composed by GRAS lipids. However, to stabilize the droplets, high concentrations of surfactants are used, and thus their toxicity and biocompatibility may be compromised.

- Easy to manufacture and scale up, although the production methods can be expensive.

- In comparison to liposomes are more stable and provide higher encapsulation efficiency than lipophilic drugs.

- $\quad$ SNEDDS have higher physicochemical stability than classical nanoemulsions.

- $\quad$ SNEOFs promote lymphatic absorption by inhibiting first-pass metabolism and

P-glycoprotein (P-gp) efflux, resulting in the complete eradication of HIV in lymphatic reservoirs.

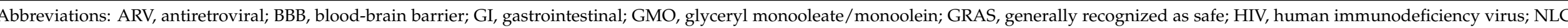

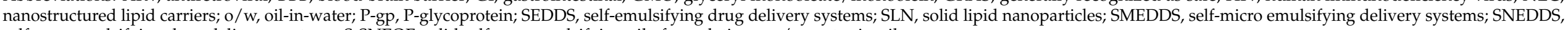
self-nanoemulsifying drug delivery systems; S-SNEOF, solid self-nanoemulsifying oily formulations; w/o, water-in-oil. 
Because of their nanoscale dimensions and adjustable surface properties, lipid-based nanocarriers are frequently advantageous for delivering antivirals to affected areas [67]. Several earlier studies have proven the potential of lipid-based nanocarriers to encapsulate and transport ARV. Representative examples are presented in Tables 3-5, highlighting the structure, composition, and physicochemical properties of nanocarriers, as well as the main challenges that were overcome by their use. Generally speaking, we may say that appropriate nanocarriers for drug delivery may be able to compensate for ARD drugs' limitations and increase their pharmacological efficacy. One goal shared by all studies is the development of nanocarriers to reduce the side effects of ARD drugs. The concentration of AZT inside red blood cells, for example, caused hematopoietic toxicity. Liposomes containing AZT decreased blood cell absorption, overcoming AZT's negative hematopoietic effects $[57,68]$ (Table 3 ). Liposomes have also been shown to reduce ddI systemic exposure [69] while providing the same therapeutic effect as free NVP at lower doses (and thus with less toxicity) (Table 3) [70]. Furthermore, NLC containing EFV had lower toxicity when compared to the free drug (Table 5) [54]. Lipid nanocarriers can also be designed to improve drug bioavailability and prolong release thereby extending the dose interval. Because of their solid lipid matrix, SLN was the most capable of providing sustained release of the ARV encapsulated, reducing the frequency of administration. For example, the encapsulation in SLN of LPV, d4T, SQV, EFV, DRV, and AZT [64,71-76] provided a sustained release of this ARV even in the case of more hydrophilic drugs such as AZT $(\log P=0.5)$ and $\mathrm{d} 4 \mathrm{~T}(\log \mathrm{P}=0.72)$. All PI ARV (Figure 6) are cytochrome P450 $3 \mathrm{~A}$ substrates, which explains why most of them have poor pharmacokinetic features, such as extensive pre-systemic first-pass metabolism and short elimination half-lives [77]. There is also evidence that PI intracellular concentrations are influenced by P-gp and/or the activity of other efflux transporters. Moreover, some other ARV drugs inhibitors of RT have also documented short plasma half-lives (e.g., d4T, AZT, ddI) [78] which reduce their target tissue distribution. The ability of lipid nanocarriers to mediate the ARV distribution and increase their half-lives is therefore an important advantage. For example, liposomes have been documented as capable to increase half-lives of d4T, AZT, ddI, and RTV $[13-16,20,79,80]$ (Table 3). Finally, many ARTs have a limited bioavailability in the brain, but the ability of lipid nanocarriers to mediate the brain delivery of ARVs has been widely documented, either for liposomes that potentially improve brain accumulation of AZT [81], or for SLN used for improving brain bioavailability of ATV, SQV, EFV, NVP and DRV [64,65,82-85], or NLC used as carriers of LPV, ATV, ETR [83,86,87] and NE improving brain accumulation of SQV and IDV [88-90]. From these studies it is worthwhile highlighting the SLN developed for EFV delivery that attained 150 folds more brain targeting delivery than the free drug [84]; the NLC for ATV delivery that attained 2.75 folds higher $\mathrm{C}_{\max }$ at the brain and 4 folds higher brain bioavailability [86] and NE as a carrier of IDV that assured specific brain accumulation of the drug [89] (Tables 3-5). 
Table 3. Liposomes, ethosomes, cubosomes, and hybrid liposomal nanocarriers for ARV delivery.

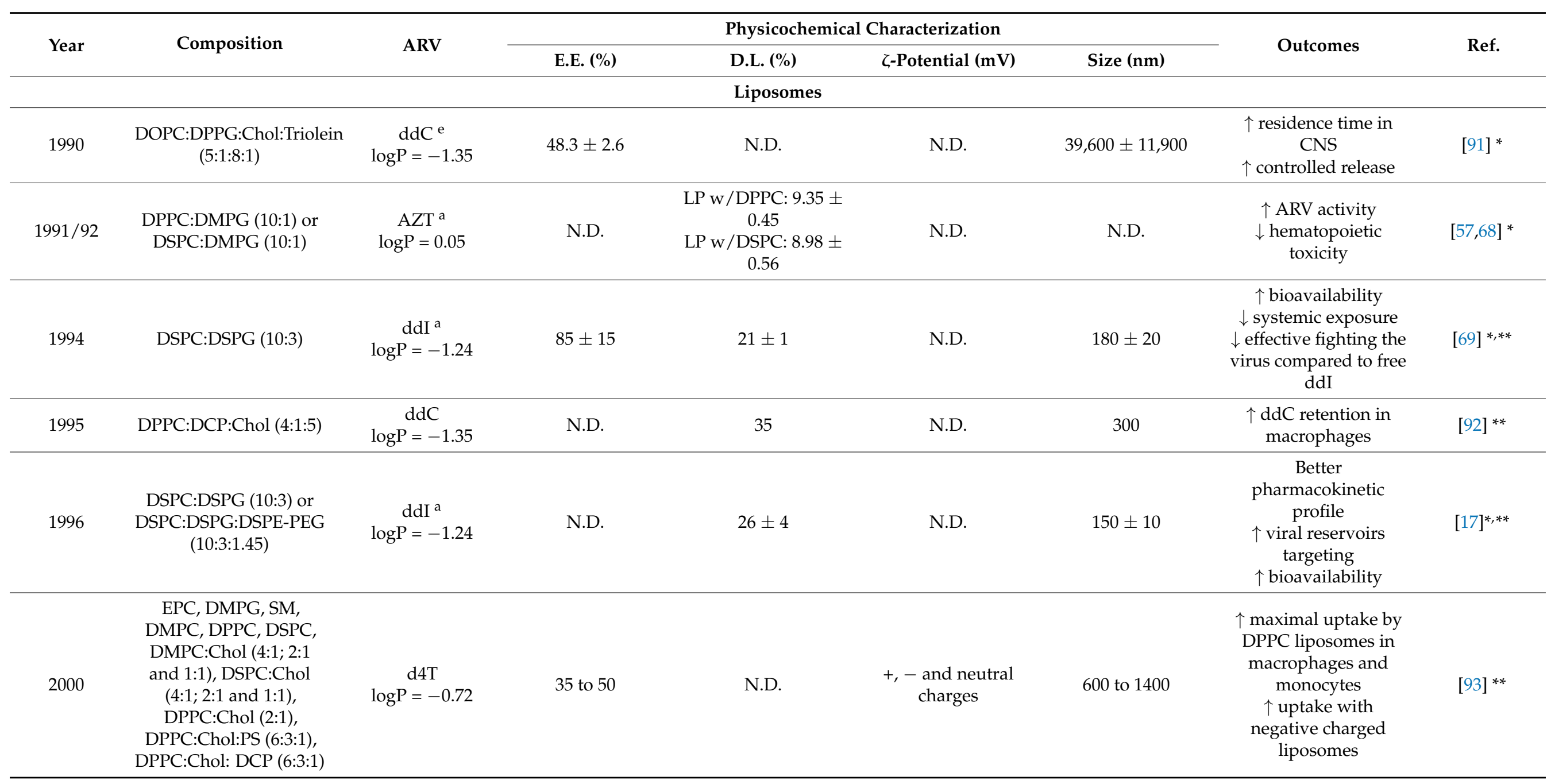


Table 3. Cont

\begin{tabular}{|c|c|c|c|c|c|c|c|c|}
\hline \multirow{2}{*}{ Year } & \multirow{2}{*}{ Composition } & \multirow{2}{*}{ ARV } & \multicolumn{4}{|c|}{ Physicochemical Characterization } & \multirow{2}{*}{ Outcomes } & \multirow{2}{*}{ Ref. } \\
\hline & & & E.E. $(\%)$ & D.L. (\%) & $\zeta$-Potential (mV) & Size $(\mathrm{nm})$ & & \\
\hline 2002 & $\begin{array}{l}\text { DPPC:DPPG:DSPE- } \\
\text { PEG-MAL } \\
(10: 3: 0.83)\end{array}$ & $\begin{array}{c}\text { IDV g } \\
\log P=2.9\end{array}$ & $11 \pm 4$ & N.D. & N.D. & 100 to 120 & $\begin{array}{c}\uparrow[\mathrm{IDV}] \text { to lymphoid } \\
\text { tissues }\end{array}$ & {$[94]^{*}$} \\
\hline 2003 & EPC:Chol (3:1) & $\begin{array}{c}\text { IDV g } \\
\log P=2.9\end{array}$ & $\begin{array}{c}97.5 \pm 2.5 \text { at } \mathrm{pH} \\
7.4 \\
\approx 20 \text { at } \mathrm{pH} 5.5\end{array}$ & $\begin{array}{l}19.5 \pm 0.5 \text { at } \mathrm{pH} 7.4 \\
\quad \approx 4 \text { at } \mathrm{pH} 5.5\end{array}$ & N.D. & $69 \pm 7$ & $\begin{array}{c}\uparrow \mathrm{CD}_{4}^{+} \mathrm{T} \text { cells } \\
\downarrow \text { viral load in lymph } \\
\text { nodes and plasma }\end{array}$ & {$[95]^{*}$} \\
\hline 2005 & SPC:AZT-M:V $\mathrm{V}_{\mathrm{E}}(6: 2: 0.1)$ & AZT-M ${ }^{a}$ & $\begin{array}{c}\text { Liophilization } \\
\text { Before: } 99.4 \pm \\
0.8 \\
\text { After: } 98.3 \pm 1.2\end{array}$ & N.D. & - charges & $\begin{array}{c}\text { Liophilization } \\
\text { Before: } 88.5 \pm 4.5 \\
\text { After: } 89.6 \pm 6.3\end{array}$ & $\begin{array}{c}\uparrow[\mathrm{ARV}] \text { in organs of } \\
\text { RES and brain }\end{array}$ & {$[81]^{*}$} \\
\hline 2006 & $\begin{array}{l}\text { EPC:Chol:SA }(7: 2: 1) \\
\text { Uncoated LP or coated } \\
\text { w/OPM: OPM-LP }\end{array}$ & $\begin{array}{c}\mathrm{d} 4 \mathrm{~T}^{\mathrm{a}} \\
\log \mathrm{P}=-0.72\end{array}$ & $\begin{array}{c}\text { Uncoated LP: } \\
49.6 \pm 1.2 \\
\text { OPM-LP: } 47.2 \pm \\
3.3\end{array}$ & N.D. & $\begin{array}{c}\text { Uncoated LP: } \\
\text { + charge } \\
\text { OPM-LP: charges } \\
\downarrow \text { close to } \\
\text { neutrality }\end{array}$ & $\begin{array}{l}\text { Uncoated LP: } \\
120 \pm 1.5 \\
\text { OPM-LP: } \\
140 \pm 2.3\end{array}$ & $\begin{array}{c}\uparrow \text { targeting } \\
\uparrow \text { residence time in } \\
\text { HIV viral reservoirs } \\
\uparrow \mathrm{d} 4 \mathrm{~T} \text { half-life } \\
\uparrow \text { pharmacological } \\
\text { activity } \\
\uparrow \text { distribution } \\
\downarrow \text { elimination }\end{array}$ & {$[13]^{*, * *}$} \\
\hline 2007 & $\begin{array}{l}\text { EPC:Chol:DMPE (7:2:1) } \\
\text { Uncoated LP or coated } \\
\text { w/OPG: OPG-LP }\end{array}$ & $\begin{array}{c}\mathrm{d} 4 \mathrm{~T}^{\mathrm{a}} \\
\log \mathrm{P}=-0.72\end{array}$ & $\begin{array}{c}\text { Uncoated LP: } \\
46.2 \pm 0.69 \\
\text { OPM-LP: } 47.1 \pm \\
1.2\end{array}$ & N.D. & $\begin{array}{l}\text { Uncoated LP: } \\
+8.21 \pm 0.15 \\
\text { OPG-LP: } \\
+3.2 \pm 0.21\end{array}$ & $\begin{array}{l}\text { Uncoated LP: } \\
122.3 \pm 0.3 \\
\text { OPM-LP: } \\
129.5 \pm 0.3\end{array}$ & $\begin{array}{c}\uparrow \mathrm{d} 4 \mathrm{~T} \text { half-life } \\
\uparrow \text { residence time } \\
\uparrow \text { hepatic cellular d4T } \\
\text { uptake }\end{array}$ & {$[14]^{*, * *, * * *}$} \\
\hline
\end{tabular}


Table 3. Cont

\begin{tabular}{|c|c|c|c|c|c|c|c|c|}
\hline \multirow{2}{*}{ Year } & \multirow{2}{*}{ Composition } & \multirow{2}{*}{ ARV } & \multicolumn{4}{|c|}{ Physicochemical Characterization } & \multirow{2}{*}{ Outcomes } & \multirow{2}{*}{ Ref. } \\
\hline & & & E.E. (\%) & D.L. (\%) & $\zeta$-Potential (mV) & Size (nm) & & \\
\hline 2008 & $\begin{array}{l}\text { EPC:Chol:DMPE }(7: 2: 1) \\
\text { Uncoated LP or coated } \\
\text { w/OPG: OPG-LP }\end{array}$ & $\begin{array}{c}\mathrm{d} 4 \mathrm{~T}^{\mathrm{a}} \\
\log \mathrm{P}=-0.72\end{array}$ & $\begin{array}{c}\text { Uncoated LP: } \\
49.6 \pm 1.2 \\
\text { OPG-LP: } 48.7 \pm \\
0.2\end{array}$ & N.D. & N.D. & $\begin{array}{l}\text { Uncoated LP: } \\
\qquad \begin{array}{l}120 \pm 4 \\
\text { OPG-LP: } \\
126 \pm 4\end{array}\end{array}$ & $\begin{array}{c}\text { Inhibition of HIV p24 } \\
\text { protein with uncoated } \\
\text { LP and OPG-LP } \\
\uparrow \text { accumulation of } \\
\text { OPG-LP in the liver, } \\
\text { spleen, and MPS } \\
\downarrow \text { uptake of OPG-LP } \\
\text { in bone }\end{array}$ & {$[96]^{*, * *}$} \\
\hline 2006 & $\begin{array}{l}\text { EPC:Chol:PE (7:2:1) } \\
\text { Uncoated LP or coated } \\
\text { w/OPG: OPG-LP) }\end{array}$ & $\begin{array}{c}\mathrm{AZT}^{\mathrm{a}} \\
\log \mathrm{P}=0.05\end{array}$ & $\begin{array}{c}\text { Uncoated LP: } \\
54.3 \pm 3.3 \\
\text { OPG-LP: } 53.9 \pm \\
2.1\end{array}$ & N.D. & $\begin{array}{c}\text { Uncoated LP: } \\
\text { + charge } \\
\text { OPM-LP: charges } \\
\downarrow \text { close to } \\
\text { neutrality }\end{array}$ & $\begin{array}{c}\text { Uncoated LP: } \\
120.0 \pm 2.1 \\
\text { OPG-LP: } \\
136.9 \pm 1.9\end{array}$ & $\begin{array}{l}\uparrow \text { AZT half-life } \\
\uparrow \text { residence time } \\
\uparrow \text { bioavailability }\end{array}$ & {$[15]^{*, * *}$} \\
\hline 2006 & $\begin{array}{l}\text { SPC:Span80 } \\
\text { SPC:PEG-8-L }(85: 15) \\
\text { (85:15) }\end{array}$ & $\begin{array}{c}\mathrm{AZT}^{\mathrm{c}} \\
\log \mathrm{P}=0.05\end{array}$ & $\begin{array}{c}\text { LP w } / \text { Span } 80^{\circledR}: \\
63.5 \pm 2.9 \\
\text { LP w } / \text { PEG-8-L: } \\
57.1 \pm 3.1\end{array}$ & N.D. & $\begin{array}{l}\text { LP w/Span } 80: \\
-2.8 \pm 0.4 \\
\text { LP w/PEG- } 8 \text {-L: } \\
-16.7 \pm 0.7\end{array}$ & $\begin{array}{c}\text { LP w/Span } 80^{\circledR}: 132 \\
\pm 15 \\
\text { LP w/PEG-8-L: } 116 \\
\pm 10\end{array}$ & $\begin{array}{c}\text { Better } \\
\text { pharmacokinetic } \\
\text { profile } \\
\uparrow \text { accumulation of } \\
\text { AZT in target RES } \\
\text { organs } \\
\uparrow \text { AZT half-life } \\
\uparrow \text { residence time, } \\
\text { targeting, and } \\
\text { controlled release }\end{array}$ & {$[19]^{*, * *}$} \\
\hline 2007 & $\begin{array}{c}\text { DPPC } \\
\text { Note: intended for oral } \\
\text { administration }\end{array}$ & $\begin{array}{c}\mathrm{ddI} \\
\log \mathrm{P}=-1.24\end{array}$ & N.D. & N.D. & N.D. & $1160 \pm 129$ & $\uparrow$ bioavailability & {$[97]^{* *}$} \\
\hline
\end{tabular}


Table 3. Cont

\begin{tabular}{|c|c|c|c|c|c|c|c|c|}
\hline \multirow{2}{*}{ Year } & \multirow{2}{*}{ Composition } & \multirow{2}{*}{ ARV } & \multicolumn{4}{|c|}{ Physicochemical Characterization } & \multirow{2}{*}{ Outcomes } & \multirow{2}{*}{ Ref. } \\
\hline & & & E.E. $(\%)$ & D.L. (\%) & $\zeta$-Potential (mV) & Size $(\mathrm{nm})$ & & \\
\hline 2007 & PC:POPG (3:1) & $\begin{array}{c}\text { IDV } \\
\log P=2.9 \mathrm{SQV} \\
\log P=3.8\end{array}$ & N.D. & N.D. & N.D. & 130 to 150 & $\begin{array}{c}\uparrow \text { liposomal } \\
\text { solubilization of both } \\
\text { drugs } \\
\uparrow \text { drug concentration } \\
\text { in the media (10- and } \\
750-\text { fold for IDV and } \\
\text { SQV, respectively) }\end{array}$ & {$[98]^{* *}$} \\
\hline 2008 & $\begin{array}{c}\text { Plain-LP: } \\
\text { SPC:PE:Span } 80 \\
(42.5: 42.5: 15) \\
\text { PEG-LP: } \\
\text { SPC:PE:Span 80:MPEG } \\
2000 \\
(42.5: 42.5: 15: 33.3) \\
\end{array}$ & $\begin{array}{c}\mathrm{AZT}^{\mathrm{c}} \\
\log \mathrm{P}=0.05\end{array}$ & $\begin{array}{c}\text { Plain-LP: } \\
63.5 \pm 2.9 \\
\text { PEG-LP: } \\
72.3 \pm 4.5\end{array}$ & N.D. & $\begin{array}{l}\text { Plain-LP: } \\
-2.8 \pm 0.4 \\
\text { PEG-LP: } \\
-18.2 \pm 0.8\end{array}$ & $\begin{array}{l}\text { Plain-LP: } \\
132 \pm 14 \\
\text { PEG-LP: } \\
158 \pm 15\end{array}$ & $\begin{array}{c}\uparrow \text { cellular uptake in } \\
\text { lymphoid cells } \\
\uparrow \text { biodistribution } \\
\uparrow \text { residence time and } \\
\text { sustained drug release }\end{array}$ & {$[53]^{*, * *}$} \\
\hline 2008 & $\begin{array}{c}\text { LP: SPC:Chol (7:3) } \\
\text { + charge LP: } \\
\text { SPC:Chol:SA (7:3:1) } \\
\text { - charge LP: } \\
\text { SPC:Chol:DCP (7:3:1) } \\
\text { w/Mannose: } \\
\text { SPC:Chol:Man (7:3:2.5) }\end{array}$ & $\begin{array}{c}\mathrm{AZT} \\
\log \mathrm{P}=0.05\end{array}$ & $\begin{array}{c}\text { LP: } \\
18.5 \pm 1.2 \\
\text { + charge LP: } \\
24.2 \pm 0.9 \\
\text { - charge LP: } \\
22.4 \pm 1.4 \\
\text { Man-LP: } 20.0 \pm \\
2.5\end{array}$ & N.D. & $\begin{array}{c}\text { LP: } \\
+10.3 \pm 1.8 \\
+ \text { charge LP: } \\
+54.4 \pm 2.3 \\
\text { - charge LP: } \\
-34.8 \pm 4.45 \\
\text { Man-LP: } \\
+14.7 \pm 3.9\end{array}$ & $\begin{array}{c}\text { LP: } 122 \pm 6 \\
\text { + charge LP: } \\
126 \pm 3 \\
\text { - charge LP: } \\
128 \pm 4 \\
\text { Man-LP: } 127 \pm 1.2\end{array}$ & $\begin{array}{c}\downarrow \text { release in Man-LP as } \\
\text { compared to LP } \\
\uparrow \text { uptake } \\
\uparrow \text { localization of } \\
\text { Man-LP in the lymph } \\
\text { nodes and spleen }\end{array}$ & {$[99]^{*, * *}$} \\
\hline 2009 & $\begin{array}{c}\text { HSPC:Chol:mPEG- } \\
\text { DSPE } \\
(55: 40: 5)\end{array}$ & PI1 & N.D. & N.D. & N.D. & N.D. & $\begin{array}{c}\uparrow \text { and longer antiviral } \\
\text { activity } \\
\text { Facilitated specific } \\
\text { uptake by } \\
\text { non-phagocytic } \\
\text { HIV-infected cells }\end{array}$ & {$[100]^{* *}$} \\
\hline
\end{tabular}


Table 3. Cont

\begin{tabular}{|c|c|c|c|c|c|c|c|c|}
\hline \multirow{2}{*}{ Year } & \multirow{2}{*}{ Composition } & \multirow{2}{*}{ ARV } & \multicolumn{4}{|c|}{ Physicochemical Characterization } & \multirow{2}{*}{ Outcomes } & \multirow{2}{*}{ Ref. } \\
\hline & & & E.E. $(\%)$ & D.L. (\%) & $\zeta$-Potential (mV) & Size $(\mathrm{nm})$ & & \\
\hline 2010 & EPC:Chol (9:1) & $\begin{array}{c}\text { NVP } \\
\log P=2.5\end{array}$ & 78.1 & 7.81 & N.D. & $<200$ & $\begin{array}{c}\uparrow \text { E.E. } \\
\text { Quick in vitro release } \\
\text { from liposomes }\end{array}$ & {$[101]^{* *}$} \\
\hline 2011 & DPPC & $\begin{array}{l}\text { ProddINP } b \\
\log P=0.05\end{array}$ & 99 & 8.83 & $-0.8 \pm 0.5$ & 187 to 208 & $\begin{array}{l}\uparrow \text { ddI blood half-life } \\
\text { (3-fold) } \\
\uparrow \text { accumulation as } \\
\text { prodrug at } 24 \mathrm{~h} \text { in } \\
\text { various organs } \\
\text { compared to plain } \\
\text { drug }\end{array}$ & {$[79]^{*, * *}$} \\
\hline 2011 & DPPC:EDPPC (1:1) & $\begin{array}{c}\text { SFV } \\
\log P=-19.5\end{array}$ & N.D. & N.D. & N.D. & N.D. & $\begin{array}{c}\text { Strong affinity of SFV } \\
\text { for DPPC:EDPPC } \\
\uparrow \text { Affinity with } \uparrow \\
\text { cationic EDPPC } \\
\text { Fusion w/viral/raft- } \\
\text { mimicking } \\
\text { vesicles }\end{array}$ & {$[102]^{* *}$} \\
\hline 2011 & $\begin{array}{c}\text { Chol:SA }(194: 1 ; 39: 1 ; \\
\text { 22:1; } 16: 1 ; 4: 1) \text { w/Span } \\
20^{\circledR} / \text { Span } 40^{\circledR} / \text { Span } \\
60^{\circledR}\end{array}$ & $\begin{array}{c}\text { TFV } \\
\log P=-1.6\end{array}$ & 3.46 to 65.26 & N.D. & +4.79 to +17.13 & 36.13 to 114.9 & $\begin{array}{c}\text { The composition had } \\
\text { a significant impact on } \\
\text { TFV release } \\
\text { Size and } \zeta \text { were } \\
\text { inversely proportional } \\
\text { to the homogenization } \\
\text { parameters, in } \\
\text { contrast to the E.E. } \\
\text { and conductivity } \\
\text { TFV distributed } \\
\text { within both the } \\
\text { aqueous and lipid } \\
\text { phases }\end{array}$ & {$[103]^{* *}$} \\
\hline
\end{tabular}


Table 3. Cont

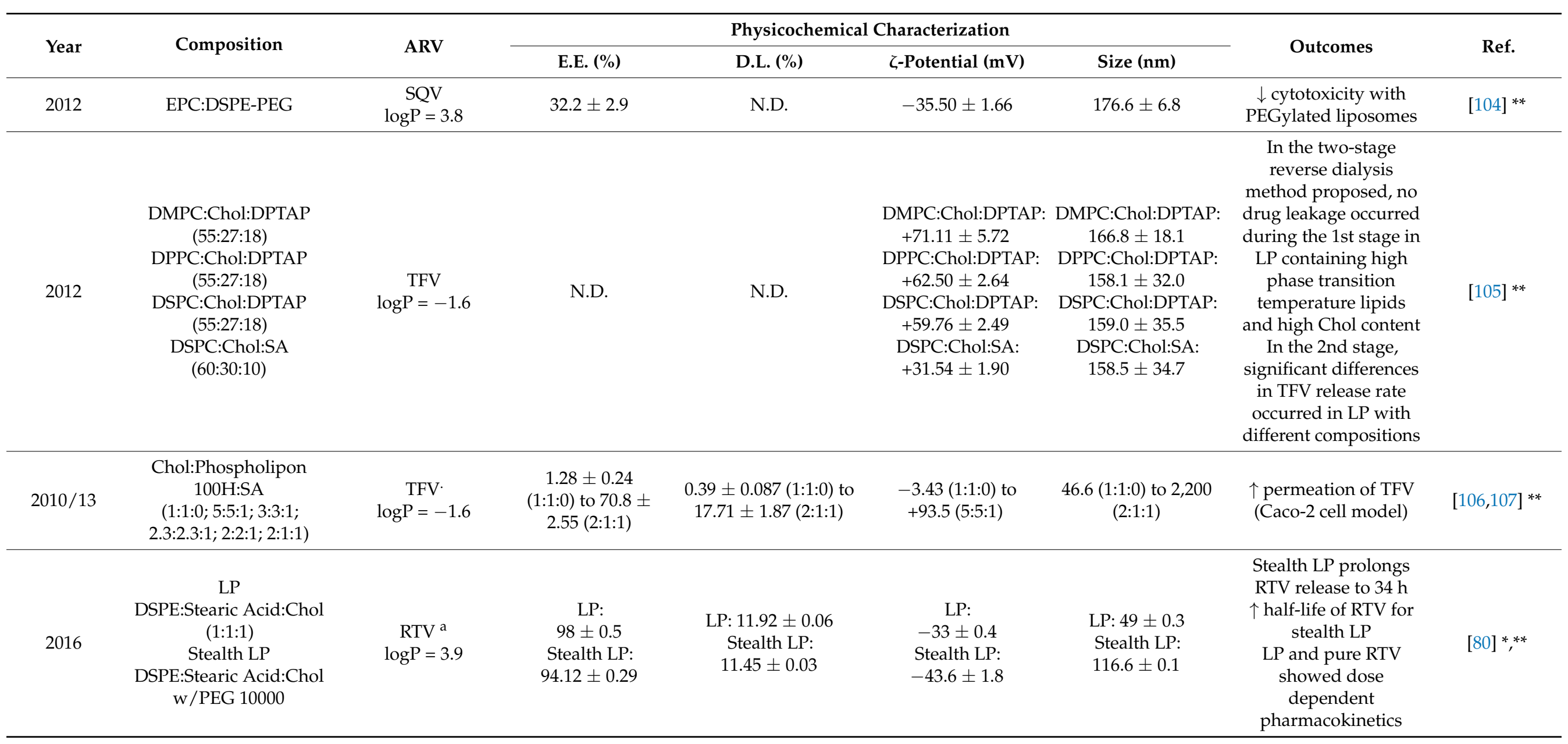


Table 3. Cont

\begin{tabular}{|c|c|c|c|c|c|c|c|c|}
\hline \multirow{2}{*}{ Year } & \multirow{2}{*}{ Composition } & \multirow{2}{*}{ ARV } & \multicolumn{4}{|c|}{ Physicochemical Characterization } & \multirow{2}{*}{ Outcomes } & \multirow{2}{*}{ Ref. } \\
\hline & & & E.E. $(\%)$ & D.L. (\%) & $\zeta$-Potential (mV) & Size $(\mathrm{nm})$ & & \\
\hline 2017 & $\begin{array}{c}\text { Phospholipon } \\
\text { 100H:Chol:SA (3:3:1 and } \\
\text { 2:2:1) } \\
\text { Note: intended for oral } \\
\text { administration }\end{array}$ & $\begin{array}{c}\text { TFV } \\
\log P=-1.6\end{array}$ & $\begin{array}{c}(3: 3: 1): \\
39.8 \pm 8.1 \\
(2: 2: 1): \\
68.1 \pm 2.6\end{array}$ & N.D. & + charge & N.D. & $\begin{array}{c}\uparrow \text { cellular permeability } \\
\text { (10 times higher) } \\
\uparrow \text { E.E. }\end{array}$ & {$[108]^{* *}$} \\
\hline 2017 & HSPC:Chol (7:3) & $\begin{array}{c}\mathrm{LPV}^{\mathrm{b}} \\
\log \mathrm{P}=5.94\end{array}$ & $90.47 \pm 0.32$ & N.D. & $-24.8 \pm 0.21$ & $659.7 \pm 23.1$ & $\begin{array}{c}\uparrow \text { LPV release at } 60 \\
\text { min }(95 \% \text { for LPV } \\
\text { loaded proliposomes } \\
\text { vs. } 55 \% \text { for free LPV) } \\
\uparrow \text { intestinal } \\
\text { permeation }(\approx 1.99 \\
\text { fold) compared to } \\
\text { pure LPV) } \\
\uparrow \text { oral bioavailability } \\
\text { (2.24- and } 1.16 \text {-fold) } \\
\text { than pure LPV and } \\
\text { commercial LPV } / \text { RTV, } \\
\text { respectively. }\end{array}$ & {$[109]^{* * * * * * *}$} \\
\hline 2015 & $\begin{array}{c}\text { EPC:Chol:DSPE-PEG } \\
(9: 1: 1)\end{array}$ & $\begin{array}{c}\text { NVP } \\
\log P=2.5 \text { and } \\
\text { SQV } \log P=3.8\end{array}$ & $\begin{array}{l}\text { NVP: } 44 \pm 2 \\
\text { SQV: } 44 \pm 1\end{array}$ & N.D. & $-29 \pm 2$ & $160 \pm 2$ & $\begin{array}{l}\text { } \uparrow \text { inhibition of viral } \\
\text { proliferation at lower } \\
\text { doses compared to } \\
\text { free drugs } \\
\text { NVP is mainly } \\
\text { released in the early } \\
\text { phases and SQV in the } \\
\text { later phases of } \\
\text { infection }\end{array}$ & {$[70]^{* *}$} \\
\hline
\end{tabular}


Table 3. Cont

\begin{tabular}{|c|c|c|c|c|c|c|c|c|}
\hline \multirow{2}{*}{ Year } & \multirow{2}{*}{ Composition } & \multirow{2}{*}{ ARV } & \multicolumn{4}{|c|}{ Physicochemical Characterization } & \multirow{2}{*}{ Outcomes } & \multirow{2}{*}{ Ref. } \\
\hline & & & E.E. $(\%)$ & D.L. (\%) & $\zeta$-Potential (mV) & Size (nm) & & \\
\hline 2017 & $\begin{array}{l}\text { SPC:Chol }(2: 1) \\
\text { Plain or coated } \\
\text { w/biotin }\end{array}$ & $\begin{array}{c}\mathrm{RTV}^{\mathrm{b}} \\
\log \mathrm{P}=3.9\end{array}$ & $\begin{array}{c}\text { Plain LP: } 62.3 \pm \\
1.7 \\
\text { Biotin-LP: } 61.6 \pm \\
1.8\end{array}$ & N.D. & $\begin{array}{l}\text { Plain LP: }-18.9 \pm \\
2.0 \\
\text { Biotin-LP: }-26.1 \\
\quad \pm 2.5\end{array}$ & $\begin{array}{c}\text { Plain LP: } 126.6 \pm 6.2 \\
\text { Biotin-LP: } 149.8 \pm \\
6.8\end{array}$ & $\begin{array}{c}\uparrow \text { release from biotin } \\
\text { coated liposomes } \\
\text { compared to } \\
\text { conventional ones } \\
\uparrow[\mathrm{RTV}] \text { in lymphatic } \\
\text { tissues }\end{array}$ & {$[110]^{*, * *}$} \\
\hline 2018 & $\begin{array}{c}\text { DSPC:DSPE-mPEG2000 } \\
(9: 1)\end{array}$ & $\begin{array}{c}\text { ATV g } \\
\log \mathrm{P}=4.5 \\
\text { RTV } \\
\log \mathrm{P}=3.9 \\
\text { TFV } \\
\log P=-1.6\end{array}$ & $\begin{array}{l}\text { ATV: } 99 \pm 8.2 \\
\text { RTV: } 92 \pm 7.1 \\
\text { TFV: } 10 \pm 0.8\end{array}$ & N.D. & N.D. & 6 to 62 & $\begin{array}{c}\uparrow \text { residence time in } \\
\text { plasma and peripheral } \\
\text { blood mononuclear } \\
\text { cells }\end{array}$ & {$[21]^{*}$} \\
\hline 2019 & $\begin{array}{c}\mathrm{DPPC} \\
\text { Note: intended for vaginal } \\
\text { administration }\end{array}$ & $\begin{array}{c}\text { TDF } \\
\log P=2.65 \\
\text { FTC } \\
\log P=-0.43\end{array}$ & 84 & 1 & Zwitterio-nic & $134 \pm 13$ & $\begin{array}{l}\uparrow \text { TDF permeation and } \\
\uparrow \text { sustained release } \\
\text { Non-cytotoxic in } \\
\text { CaSki (epidermoid } \\
\text { cervical cancer cell } \\
\text { line) and HEC-1-A } \\
\text { (Human Endo-metrial } \\
\text { Cancer-1) }\end{array}$ & {$[52]^{* *}$} \\
\hline 2020 & $\begin{array}{c}\text { POPC } \\
\text { POPC:DPPE-PEG } 2000 \\
(9: 1)\end{array}$ & $\begin{array}{c}\mathrm{T} 20 \\
\log \mathrm{P}=-14.7 \\
\text { PPI } \\
\text { xT20 + PPIX }\end{array}$ & N.D. & N.D. & $\begin{array}{c}\text { Zwitterio- } \\
\text { nic } \\
\text { charge was } \\
\text { predominantly } \\
\text { affected by PPIX }\end{array}$ & $\begin{array}{c}\text { Unloaded POPC: } \\
110 \text { nm } \\
\text { Unloaded } \\
\text { POPC:DPPE- } \\
\text { PEG }_{2000}(9: 1): \\
120 \text { nm } \\
\text { Size was affected by } \\
\text { PPIX }\end{array}$ & $\begin{array}{l}\uparrow \text { entry inhibitors (T20 } \\
\text { and PPIX) synergy } \\
\text { compared to } \\
\text { combination in free } \\
\text { aqueous form }\end{array}$ & {$[111]^{* *}$} \\
\hline
\end{tabular}


Table 3. Cont

\begin{tabular}{|c|c|c|c|c|c|c|c|c|}
\hline \multirow{2}{*}{ Year } & \multirow{2}{*}{ Composition } & \multirow{2}{*}{ ARV } & \multicolumn{4}{|c|}{ Physicochemical Characterization } & \multirow{2}{*}{ Outcomes } & \multirow{2}{*}{ Ref. } \\
\hline & & & E.E. $(\%)$ & D.L. (\%) & $\zeta$-Potential (mV) & Size (nm) & & \\
\hline \multicolumn{9}{|c|}{ Ethosomes } \\
\hline \multicolumn{9}{|c|}{ Cubosomes } \\
\hline 2021 & $\begin{array}{c}\text { GMO:CTAB:poloxamer } \\
\text { 407(245:9:1, 219:9:1) }\end{array}$ & $\begin{array}{c}\text { ATV } \\
\log P=4.5\end{array}$ & $\begin{array}{c}61 \pm 4.6(219: 9: 1) \\
\text { to } \\
93 \pm 1.2(245: 9: 1)\end{array}$ & N.D. & $\begin{array}{c}-29.41(219: 9: 1) \\
\text { to } \\
-24.53(245: 9: 1)\end{array}$ & $\begin{array}{c}253 \pm 5.6(219: 9: 1) \\
\text { to } \\
150 \pm 8.7 \\
(245: 9: 1)\end{array}$ & $\begin{array}{c}\uparrow \text { ATV absorption and } \\
\text { bioavailability (4.6 } \\
\text { folds) compared to } \\
\text { oral administration } \\
\uparrow \text { transdermal drug } \\
\text { permeation due to } \\
\text { bio-adhesive } \\
\text { characteristic and } \\
\text { permeation } \\
\text { enhancement effect }\end{array}$ & {$[113]^{*, * * * * * *}$} \\
\hline 2020 & $\begin{array}{c}\text { GMO:CTAB: poloxamer } \\
407(18: 15: 1)\end{array}$ & $\begin{array}{c}\mathrm{SQV} \text { b,d } \\
\log \mathrm{P}=3.8\end{array}$ & $\begin{array}{c}72 \pm 2 \\
\text { higher } \\
\text { concentrations } \\
\text { of GMO favored } \\
\text { drug entrapment }\end{array}$ & N.D. & N.D. & $\begin{array}{c}120 \pm 2 \\
\uparrow \text { particle size with } \\
\uparrow \text { GMO and } \downarrow \\
\text { Poloxamer } 407\end{array}$ & $\begin{array}{c}\uparrow \text { SQV bioavailability } \\
\text { (12-fold and } 2.5 \text {-fold) } \\
\text { when compared with } \\
\text { oral and intranasal } \\
\text { administration of free } \\
\text { SQV }\end{array}$ & {$[114]^{* * * *}$} \\
\hline \multicolumn{9}{|c|}{ Hybrid liposomal nanocarriers } \\
\hline 2017 & $\begin{array}{c}\text { SPC and gelatin } \\
\text { nanoparticles (SG-LP) }\end{array}$ & $\begin{array}{c}\mathrm{d} 4 \mathrm{~T} \\
\log \mathrm{P}=-0.72\end{array}$ & $\begin{array}{c}\text { Gelatin NP (SG): } \\
56.0 \pm 1.7 \\
\text { SG-LP: } 55.1 \pm \\
2.1\end{array}$ & N.D. & $\begin{array}{c}\text { SG-LP: } \\
-44.6 \pm 1.36\end{array}$ & $\begin{array}{c}\text { SG-LP: } \\
232.9 \pm 1.5\end{array}$ & $\begin{array}{c}\uparrow \text { controlled release } \\
\uparrow \text { uptake and } \\
\text { hemocompatibility } \\
\uparrow \mathrm{d} 4 \mathrm{~T} \text { half-life } \\
\downarrow \text { blood viremia }\end{array}$ & {$[20]^{* *}$} \\
\hline
\end{tabular}


Table 3. Cont

\begin{tabular}{|c|c|c|c|c|c|c|c|c|}
\hline \multirow{2}{*}{ Year } & \multirow{2}{*}{ Composition } & \multirow{2}{*}{ ARV } & \multicolumn{4}{|c|}{ Physicochemical Characterization } & \multirow{2}{*}{ Outcomes } & \multirow{2}{*}{ Ref. } \\
\hline & & & E.E. (\%) & D.L. (\%) & $\zeta$-Potential (mV) & Size (nm) & & \\
\hline 2017 & $\begin{array}{c}\text { LP } \\
\text { DPPC or DPPC:Chol } \\
\text { (1:1, 4:1, 2:1) } \\
\text { Magneto-plasmonic LP } \\
\text { MNP@Au coated } \\
\text { w/PEG }\end{array}$ & $\begin{array}{c}\text { TDF } \\
\log P=2.65\end{array}$ & $\begin{array}{c}\uparrow \text { E.E. } w / \text { higher } \\
\text { drug ratio } \\
(\approx 30 \% \text { for } \\
\text { LP:TDF }(1: 34)) \\
\uparrow \text { E.E. w/smaller } \\
\text { Chol content } \\
(\approx 60 \% \text { for } \\
\text { DPPC) }\end{array}$ & N.D. & N.D. & $\begin{array}{c}\downarrow \text { with increasing } \\
\text { Chol }\end{array}$ & $\begin{array}{c}\uparrow \text { TDF release for LP } \\
\text { without Chol } \\
\uparrow \text { transmigration } \\
\text { across an in vitro BBB } \\
\text { model by magnetic } \\
\text { targeting } \\
\downarrow \text { viral replication of } \\
\text { HIV infected } \\
\text { microglial cells }\end{array}$ & {$[115]^{* *}$} \\
\hline 2010 & $\begin{array}{c}\text { LP } \\
\text { SPC:Chol (1.2:1) } \\
\text { Magnetic LP } \\
\text { LP + magnetic AZTTP } \\
\text { NP }\end{array}$ & AZTTP & $54.5 \pm 6$ & N.D. & N.D. & $\sim 150 \mathrm{~nm}$ & $\begin{array}{c}\uparrow \text { permeability (3-fold) } \\
\text { for magnetic AZTTP } \\
\text { LP than free AZTTP } \\
\text { Efficient taken up by } \\
\text { monocytes } \\
\uparrow \text { transendothelial } \\
\text { migration in presence } \\
\text { of an external } \\
\text { magnetic field } \\
\text { compared to } \\
\text { normal/non- } \\
\text { magnetic } \\
\text { monocytes }\end{array}$ & {$[116]^{* *}$} \\
\hline
\end{tabular}


Table 3. Cont.

\begin{tabular}{|c|c|c|c|c|c|c|c|c|}
\hline \multirow{2}{*}{ Year } & \multirow{2}{*}{ Composition } & \multirow{2}{*}{ ARV } & \multicolumn{4}{|c|}{ Physicochemical Characterization } & \multirow{2}{*}{ Outcomes } & \multirow{2}{*}{ Ref. } \\
\hline & & & E.E. (\%) & D.L. (\%) & $\zeta$-Potential (mV) & Size (nm) & & \\
\hline 2021 & $\begin{array}{c}\text { LP } \\
\text { DMPC:DOPE:Chol } \\
\text { (7:2:1) } \\
\text { inside PVA nanofibers }\end{array}$ & $\begin{array}{c}\mathrm{TDF}^{\mathrm{h}} \\
\log \mathrm{P}=2.65 \\
\text { FTC } \\
\log \mathrm{P}=-0.43\end{array}$ & 100 & 4 (FTC) and 2.8 (TDF) & $\begin{array}{c}\mathrm{LP} \\
-0.67 \pm 0.01\end{array}$ & $211 \pm 24$ & $\begin{array}{l}\text { Rapid onset of local } \\
\text { drug levels upon } \\
\text { single vaginal } \\
\text { administration of } \\
\text { fibers to mice } \\
\text { comparing to the } \\
\text { continuous daily use } \\
\text { for } 5 \text { days of oral } \\
\text { TDF/FTC } \\
\text { Drug concentrations } \\
\text { in vaginal fluids were } \\
\text { fairly sustained up to } \\
\text { 1-4 h, which could be } \\
\text { translatable into a } \\
\text { quite wide protection } \\
\text { time window in } \\
\text { humans }\end{array}$ & {$[117]$ * } \\
\hline
\end{tabular}

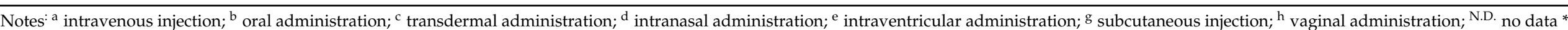

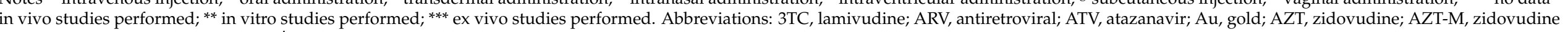

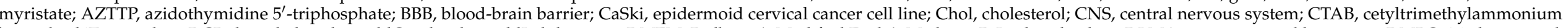

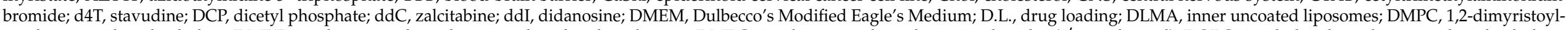

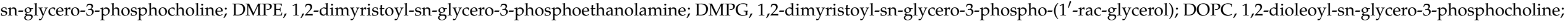

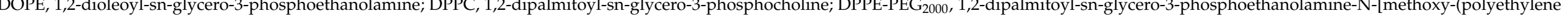

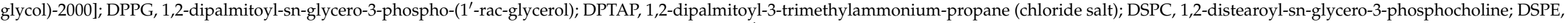

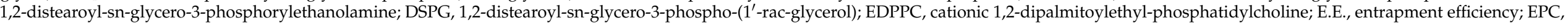

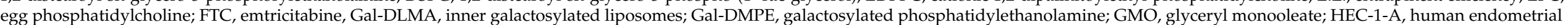

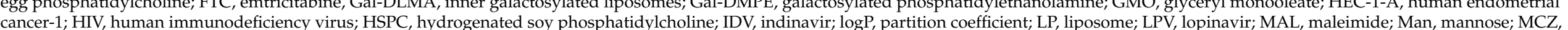

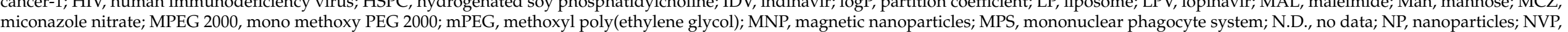

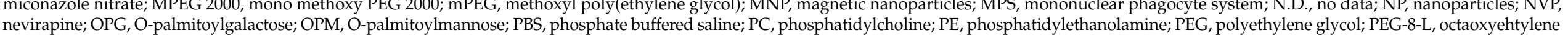

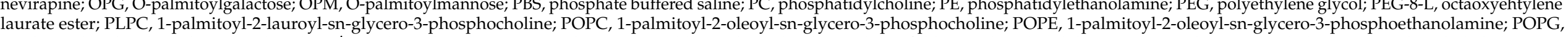

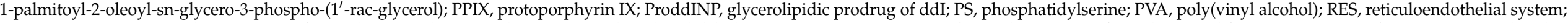

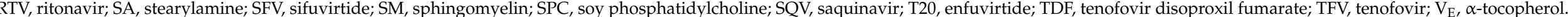


Table 4. Lipid nanoparticles (SLN and NLC) for ARV delivery.

\begin{tabular}{|c|c|c|c|c|c|c|c|c|}
\hline \multirow[b]{2}{*}{ Year } & \multirow{2}{*}{ Composition } & \multirow[b]{2}{*}{ ARV } & \multicolumn{4}{|c|}{ Physicochemical Characterization } & \multirow{2}{*}{ Outcomes } & \multirow[b]{2}{*}{ Ref. } \\
\hline & & & E.E. (\%) & D.L. (\%) & $\begin{array}{l}\zeta \text {-Potential } \\
(\mathrm{mV})\end{array}$ & Size $(\mathrm{nm})$ & & \\
\hline \multicolumn{9}{|l|}{ SLN } \\
\hline 1998 & $\begin{array}{l}\text { Lipid phase: } \\
\text { Trilaurin:DPPC:DMPG } \\
\text { (0.69:0.28: } 0.03 \mathrm{wt}: \mathrm{wt}) \\
\text { SLN coated w/PE-PEG } 2000 \\
\text { [10\% (mol ratio)] }\end{array}$ & $\begin{array}{l}\mathrm{AZT}^{\mathrm{a}} \\
\log \mathrm{P}=0.05\end{array}$ & N.D. & N.D. & $\begin{array}{l}\text { Plain SLN: } \\
-20 \pm 5 \\
\text { PE-PEG SLN: } \\
-6 \pm 4\end{array}$ & $\begin{array}{l}\text { Plain SLN: } \\
183 \pm 48 \\
\text { PE-PEG SLN: } 182 \pm 44\end{array}$ & $\begin{array}{l}\downarrow \text { release rate in } \\
\text { SLN-PE-PEG } \\
\uparrow \text { bioavailability } \\
\uparrow \text { accumulation of SLN in } \\
\text { the liver }\end{array}$ & {$[18]^{*, * *}$} \\
\hline 2006 & $\begin{array}{l}\text { Lipid phase: Trilaurin or } \\
\text { Tristearin:Chol:PC:SA } \\
\text { (1:0.5:1:0.1) }\end{array}$ & $\begin{array}{l}\mathrm{AZT}^{\mathrm{a}} \\
\log \mathrm{P}=0.05\end{array}$ & $\begin{array}{l}\text { Trilaurin SLN: } \\
57.8 \pm 6.2 \\
\text { Tristearin SLN: } \\
59.7 \pm 6.1\end{array}$ & N.D. & +charges & $\begin{array}{l}\text { Trilaurin SLN: } \\
130 \pm 18 \\
\text { Tristearin SLN: } \\
142 \pm 22\end{array}$ & $\begin{array}{l}\uparrow \text { uptake in hepatocytes } \\
\uparrow \text { controlled release } \\
(12-15 \% \text { in } 24 \mathrm{~h})\end{array}$ & {$[76]^{*, * *}$} \\
\hline 2008 & $\begin{array}{l}\text { Lipid phase: stearic acid } \\
\text { Aqueous phase: Pluronic } \\
\text { F68 ( } 3 \%) \\
\text { Note: intended for enhanced } \\
\text { brain delivery }\end{array}$ & $\begin{array}{l}\text { ATV } \\
\log P=4.5\end{array}$ & $\begin{array}{l}98.9 \pm 0.8 \\
98.2 \pm 1.3 \\
89.3 \pm 2.7\end{array}$ & $\begin{array}{l}1 \\
2 \\
5\end{array}$ & $18.43 \pm 0.70$ & $167 \pm 8.3$ & $\begin{array}{l}\text { Burst ATV release of } \approx 17 \% \\
\text { by } 1 \mathrm{~h} \text { and gradual release } \\
\text { up to } 40 \% \text { by } 24 \mathrm{~h} \\
\uparrow \text { uptake and } \\
\text { accumulation of ATV } \\
\text { when delivered by SLN } \\
\text { (human brain endothelial } \\
\text { cell monolayer) compared } \\
\text { to the free ATV }\end{array}$ & {$[65]^{* *}$} \\
\hline 2011 & $\begin{array}{l}\text { Lipid phase: Compritol }{ }^{\circledR} \\
888 \text { ATO/tripalmitin/cacao } \\
\text { butter (wt of } 8 \%) \\
\text { Aqueous phase: PC }(7 \%) \text {, } \\
\text { cholesteryl hemisuccinate } \\
(5 \%) \text {, taurocholate }(2.5 \%) \\
\text { and 1-butanol }(9.2 \%)\end{array}$ & $\begin{array}{l}\mathrm{d} 4 \mathrm{~T} \\
\log \mathrm{P}=-0.72 \\
\mathrm{DLV} \\
\log \mathrm{P}=2.8 \\
\mathrm{SQV} \\
\log \mathrm{P}=3.8\end{array}$ & $\mathrm{SQV}>\mathrm{DLV}>\mathrm{d} 4 \mathrm{~T}$ & N.D. & N.D. & $\begin{array}{l}\text { 142-308 } \\
\uparrow \% \text { Compritol }{ }^{\circledR} 888 \\
\text { ATO: } \uparrow \text { d4T-SLN mean } \\
\text { size and } \downarrow \text { DLV-SLN } \\
\text { and SQV-SLN mean size }\end{array}$ & $\begin{array}{l}\uparrow \text { E.E. for d } 4 \mathrm{~T} \\
\text { Sustained drug release: } \\
\mathrm{d} 4 \mathrm{~T}>\mathrm{DLV}>\mathrm{SQV}\end{array}$ & {$[74]^{* *}$} \\
\hline
\end{tabular}


Table 4. Cont.

\begin{tabular}{|c|c|c|c|c|c|c|c|c|}
\hline \multirow[b]{2}{*}{ Year } & \multirow[b]{2}{*}{ Composition } & \multirow[b]{2}{*}{ ARV } & \multicolumn{4}{|c|}{ Physicochemical Characterization } & \multirow[b]{2}{*}{ Outcomes } & \multirow[b]{2}{*}{ Ref. } \\
\hline & & & E.E. $(\%)$ & D.L. (\%) & $\begin{array}{l}\zeta \text {-Potential } \\
(\mathrm{mV})\end{array}$ & Size $(\mathrm{nm})$ & & \\
\hline 2011 & $\begin{array}{l}\text { Lipid phase: Compritol }{ }^{\circledR} \\
888 \text { ATO } \\
\text { Aqueous phase: } \text { Pluronic }^{\circledR} \\
\text { F127 }(2.5 \%)\end{array}$ & $\begin{array}{l}\mathrm{LPV} \text { b,f } \\
\log \mathrm{P}=5.94\end{array}$ & $>99$ & N.D. & $-26.5 \pm 0.45$ & $230.4 \pm 5.6$ & $\begin{array}{l}\text { Slow-release in both } \\
\text { media pH } 6.8 \text { and } \mathrm{pH} 1.2 \\
\uparrow \text { bioavailability and } \\
\text { targeting } \\
\uparrow \% \text { LPV secreted into the } \\
\text { lymph }\end{array}$ & {$[71]^{*, * *}$} \\
\hline 2011 & $\begin{array}{l}\text { Lipid phase: Softisan }{ }^{\circledR} 100 \\
\text { Aqueous phase: BSA and } \\
\text { PAA (negative moiety) } \\
\text { Additional layer of PLL } \\
\text { (positively charged) and } \\
\text { heparin (negatively } \\
\text { charged) } \\
\text { Note: intended for } \\
\text { topical/vaginal administration }\end{array}$ & $\begin{array}{l}\mathrm{TFV} \\
\log \mathrm{P}=-1.6\end{array}$ & $8.3 \pm 0.7$ & 0.083 & $-51.07 \pm 4.44$ & $153.66 \pm 11.33$ & $\begin{array}{l}\text { Non-cytotoxic (human } \\
\text { vaginal epithelial cell line) } \\
\text { and easily functionalized } \\
\uparrow \text { solubility }\end{array}$ & {$[118]^{* *}$} \\
\hline
\end{tabular}


Table 4. Cont.

\begin{tabular}{|c|c|c|c|c|c|c|c|c|}
\hline \multirow{2}{*}{ Year } & \multirow{2}{*}{ Composition } & \multirow{2}{*}{ ARV } & \multicolumn{4}{|c|}{ Physicochemical Characterization } & \multirow{2}{*}{ Outcomes } & \multirow{2}{*}{ Ref. } \\
\hline & & & E.E. $(\%)$ & D.L. (\%) & $\begin{array}{l}\zeta \text {-Potential } \\
(\mathrm{mV})\end{array}$ & Size (nm) & & \\
\hline 2011 & $\begin{array}{l}\text { Lipid phase: Compritol }{ }^{\circledR} \\
888 \text { ATO/steric acid }(4 \%) \\
\text { Aqueous phase: DODAB } \\
(1.8 \%) \text {, Tween }{ }^{\circledR} 80(1 \%), \\
\text { lecithin }(0.2 \%) \text { and } \\
\text { 1-butanol }(0.5 \%) \\
\text { SLN coated with HSA }\end{array}$ & $\begin{array}{l}\text { NVP } \\
\log P=2.5\end{array}$ & $\begin{array}{l}\text { NVP-SLN: } \\
\approx 77 \text { (maximum } \\
\text { achieved) }\end{array}$ & N.D. & $\begin{array}{l}\text { NVP-SLN: }+ \\
\uparrow[\text { HSA }] \downarrow \zeta \text { in } \\
\text { SLN to values } \\
\text { close to } \\
\text { neutrality }\end{array}$ & $\begin{array}{l}\text { NVP-SLN: } \\
153.1 \\
\text { HSA/NVP-SLN: } 189.2\end{array}$ & $\begin{array}{l}\uparrow \text { E.E. with SLN } \\
\downarrow \text { HBMECs viability with } \\
\text { NVP-SLN } \\
\uparrow \text { HBMECs viability with } \\
\text { HSA-NVP-SLN }\end{array}$ & {$[120]^{* *}$} \\
\hline 2013 & 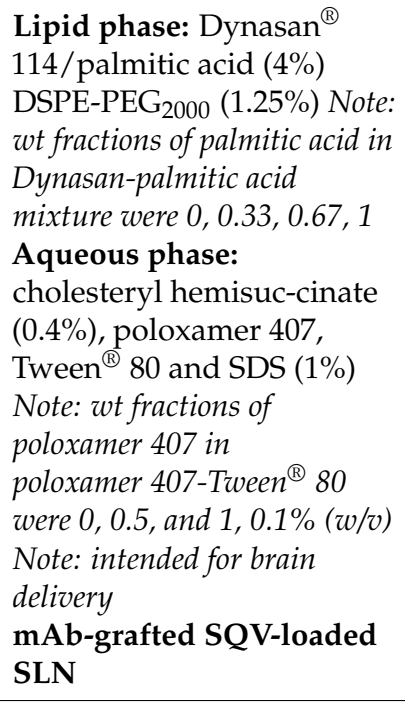 & $\begin{array}{l}\mathrm{SQV} \\
\log \mathrm{P}=3.8\end{array}$ & $\approx 55$ to 80 & N.D. & $>-30$ & 120 to 450 & $\begin{array}{l}\uparrow \text { BBB permeation } \\
\uparrow \text { bioavailability and } \uparrow \\
\text { solubility } \\
\uparrow \text { controlled release } \\
\uparrow \text { E.E. } \\
\text { High biocompatibility of } \\
\text { mAb-grafted SLN to } \\
\text { HBMECs } \uparrow \text { HBMECs } \\
\text { uptake } \\
\downarrow \text { lymphatic uptake }\end{array}$ & {$[64]^{* *}$} \\
\hline 2014 & $\begin{array}{l}\text { Lipid phase: GMS } \\
\text { Aqueous phase: Tween }{ }^{\circledR} \\
80(1.25 \%)\end{array}$ & $\begin{array}{l}\mathrm{EFV}^{\mathrm{b}} \\
\log \mathrm{P}=4.6\end{array}$ & 86 & N.D. & -15.9 & $124.5 \pm 3.2$ & $\begin{array}{l}\uparrow 5.32 \text {-fold in } C_{\max } \text { and } \uparrow \\
\text { 10.98-fold in AUC } \\
\text { w / EFV-SLN compared to } \\
\text { EFV suspension }\end{array}$ & {$[16]^{*}$} \\
\hline
\end{tabular}


Table 4. Cont.

\begin{tabular}{|c|c|c|c|c|c|c|c|c|}
\hline \multirow[b]{2}{*}{ Year } & \multirow{2}{*}{ Composition } & \multirow[b]{2}{*}{ ARV } & \multicolumn{4}{|c|}{ Physicochemical Characterization } & \multirow[b]{2}{*}{ Outcomes } & \multirow[b]{2}{*}{ Ref. } \\
\hline & & & E.E. $(\%)$ & D.L. (\%) & $\begin{array}{l}\text { c-Potential } \\
(\mathrm{mV})\end{array}$ & Size $(\mathrm{nm})$ & & \\
\hline 2015 & $\begin{array}{l}\text { Lipid phase: tristearin: } \\
\text { HSPC:DSPE (11.2:12.6:0.3, } \\
\text { molar ratio) } \\
\text { Aqueous phase: Tween }{ }^{\circledR} \\
80(0.5 \%) \\
\text { SLN conjugated with PA }\end{array}$ & $\begin{array}{l}\mathrm{EFV}^{\mathrm{a}} \\
\log \mathrm{P}=4.6\end{array}$ & $\begin{array}{l}\text { SLN: } \\
72.1 \pm 0.4 \\
\text { PA-SLN: } \\
63.5 \pm 0.6\end{array}$ & N.D. & $\begin{array}{l}\text { SLN: } \\
-28.8 \pm 1.2 \\
\text { PA-SLN: } \\
-36.2 \pm 1.0\end{array}$ & $\begin{array}{l}\text { SLN: } 113 \pm 0.2 \\
\text { PA-SLN: } 163 \pm 0.5\end{array}$ & $\begin{array}{l}\text { Able to permeate BBB } \\
\uparrow \text { bioavailability } \\
\uparrow \text { controlled release }\end{array}$ & {$[55]^{*, * *}$} \\
\hline 2015 & $\begin{array}{l}\text { Lipid phase: Gelucire }{ }^{\circledR} \\
44 / 14(30 \%) \text { and } \\
\text { Compritol }^{\circledR} 888 \text { ATO }(20 \%) \\
\text { Aqueous phase: Lipoid }{ }^{\circledR} \mathrm{S} \\
75(25 \%) \text { and poloxamer } 188 \\
(5 \%)\end{array}$ & $\begin{array}{l}\mathrm{EFV}^{\mathrm{b}} \\
\log \mathrm{P}=4.6\end{array}$ & 85.6 & 39.4 & -35.55 & $168.92 \pm 31.2$ & $\begin{array}{l}\text { Prolonged and biphasic in } \\
\text { PBS pH } 6.8 \\
\uparrow \text { bioavailability } \\
\uparrow[\mathrm{EFV}] \text { in the spleen } \\
\uparrow \text { biodistribution in } \\
\text { lymphatic organs }\end{array}$ & {$[75]^{*, * *}$} \\
\hline 2016 & $\begin{array}{l}\text { Lipid phase: stearic acid } \\
\text { Aqueous phase: PVA } \\
\text { SLN modified with Aloe } \\
\text { Vera }\end{array}$ & $\begin{array}{l}\mathrm{AZT} \\
\log \mathrm{P}=0.05\end{array}$ & $\begin{array}{l}\text { SLN: } \\
66.5 \\
\text { SLN-AV: } 84\end{array}$ & $\begin{array}{l}\text { SLN: } \\
18.01 \\
\text { SLN-AV: } \\
29.6\end{array}$ & $\begin{array}{l}\text { AZT-SLN: } \\
-12.18 \text { to } 13.1 \\
\text { AZT-SLN-AV: } \\
-14.2 \text { to } 15.41\end{array}$ & $\begin{array}{l}\text { SLN: } 222 \text { to } 227 \\
\text { SLN-AV: } 402 \text { to } 434\end{array}$ & $\begin{array}{l}\uparrow \text { solubility } \\
\uparrow \text { cellular uptake and no } \\
\text { cytotoxicity (C6 glioma } \\
\text { cells) } \\
\uparrow \text { E.E. }\end{array}$ & {$[121]^{* *}$} \\
\hline 2017 & $\begin{array}{l}\text { Lipid phase: GC and GMS } \\
(1.5 \mathrm{~g}) \\
\text { Note: N.D. of GC:GMS } \\
\text { Span } 80(1 \%) \\
\text { Aqueous phase: Tween }{ }^{\circledR} \\
80(2 \%)\end{array}$ & $\begin{array}{l}\mathrm{DRV} \\
\log \mathrm{P}=1.89\end{array}$ & $\begin{array}{l}\text { SLN: } 74.23 \\
\text { Freeze-dried SLN: } \\
69.8\end{array}$ & $\begin{array}{l}\text { Post-freeze } \\
\text { drying: } \\
9.37\end{array}$ & $\begin{array}{l}\text { Freeze-dried } \\
\text { SLN: }-22 \pm 2\end{array}$ & $\begin{array}{l}\text { SLN: } 210 \\
\text { Freeze-dried SLN: } 270\end{array}$ & $\begin{array}{l}\text { Sustained release of DRV } \\
\text { until } 12 \mathrm{~h} \\
\text { Apparent permeability } \\
\text { across rat intestine: } 24 \times \\
10^{-6}(\mathrm{~cm} / \mathrm{s}) \text { at } 37^{\circ} \mathrm{C} \text { and } \\
5.6 \times 10^{-6}(\mathrm{~cm} / \mathrm{s}) \text { at } 4^{\circ} \mathrm{C} \\
\text { Endocytic uptake }\end{array}$ & {$[73]^{* * * * * *}$} \\
\hline
\end{tabular}


Table 4. Cont.

\begin{tabular}{|c|c|c|c|c|c|c|c|c|}
\hline \multirow[b]{2}{*}{ Year } & \multirow[b]{2}{*}{ Composition } & \multirow[b]{2}{*}{ ARV } & \multicolumn{4}{|c|}{ Physicochemical Characterization } & \multirow[b]{2}{*}{ Outcomes } & \multirow[b]{2}{*}{ Ref. } \\
\hline & & & E.E. (\%) & D.L. (\%) & $\begin{array}{l}\zeta \text {-Potential } \\
(\mathrm{mV})\end{array}$ & Size $(\mathrm{nm})$ & & \\
\hline 2017 & $\begin{array}{l}\text { Lipid phase: tripalmitin } \\
\text { Aqueous phase: poloxamer } \\
188(1 \%)\end{array}$ & $\begin{array}{l}E F V^{d} \\
\log P=4.6\end{array}$ & 64.9 & N.D. & -21.2 & 108.3 & $\begin{array}{l}\text { Burst release followed by } \\
\text { a prolonged release } \\
\uparrow[\mathrm{EFV}] \text { in brain } \\
\uparrow \text { brain targeting } \\
\text { efficiency (more } 150 \text { times) } \\
\text { and better absorption of } \\
\text { the EFV (70 times more) } \\
\text { with intranasal as } \\
\text { compared to orally } \\
\text { administered marketed } \\
\text { formulation (capsule) }\end{array}$ & {$[84]^{*, * *}$} \\
\hline 2017 & $\begin{array}{l}\text { Lipid phase: GMS:SL }(1: 1) \\
\text { Aqueous phase: } \\
\text { Poloxamer118 }(0.5 \%) \text { or } \\
\text { Tween }{ }^{\circledR} 80(0.25 \%)\end{array}$ & $\begin{array}{l}\text { RTV } \\
\log \mathrm{P}=3.9\end{array}$ & $21.4-53.3$ & N.D. & $\begin{array}{l}-39.35 \pm 1.2 \text { to } \\
-50.80 \pm 4.8\end{array}$ & 178 to 254 & $\begin{array}{l}\uparrow \text { E.E. and mean size } \\
\text { using poloxamer } 118 \text { as } \\
\text { surfactant } \\
\uparrow \text { controlled release } \\
\text { RTV-SLN can maintain } \\
\text { inhibition of virus } \\
\text { production }\end{array}$ & {$[122]^{* *}$} \\
\hline 2017 & $\begin{array}{l}\text { Preconcentrate: PGDS or } \\
\text { stearic acid } \\
\text { and poloxamer } 118 \\
(0.1-1.0 \%) \\
\text { Diluted with water }\end{array}$ & $\begin{array}{l}\mathrm{NVP}{ }^{\mathrm{a}} \\
\log \mathrm{P}=2.5\end{array}$ & $>90$ & N.D. & $\begin{array}{l}\text { PGDS-SLN: } \\
-26.8 \pm 2.1\end{array}$ & $\begin{array}{l}\text { Stearic acid-SLN: } 940.2 \\
\pm 1.54 \\
\text { PGDS-SLN: } \\
70 \text { to } 1100 \text { (average } \\
\text { particle size } \sim 212 \mathrm{~nm} \text { ) }\end{array}$ & $\begin{array}{l}\text { Biphasic release profile } \\
\text { with an initial burst } \\
\text { release } \\
\uparrow[\mathrm{NVP}] \text { in the liver, } \\
\text { kidneys, and brain } \\
\text { Targeting for multiple } \\
\text { viral reservoirs }\end{array}$ & {$[85]^{*, * *}$} \\
\hline
\end{tabular}


Table 4. Cont.

\begin{tabular}{|c|c|c|c|c|c|c|c|c|}
\hline \multirow[b]{2}{*}{ Year } & \multirow{2}{*}{ Composition } & \multirow[b]{2}{*}{ ARV } & \multicolumn{4}{|c|}{ Physicochemical Characterization } & \multirow[b]{2}{*}{ Outcomes } & \multirow[b]{2}{*}{ Ref. } \\
\hline & & & E.E. $(\%)$ & D.L. (\%) & $\begin{array}{l}\text { C-Potential } \\
(\mathrm{mV})\end{array}$ & Size $(\mathrm{nm})$ & & \\
\hline 2018 & $\begin{array}{l}\text { Lipid phase: Compritol }{ }^{\circledR} \\
\text { ATO } 888(0.5 \%) \\
\text { Aqueous phase: poloxamer } \\
407(0.25 \%) \text {, Labrasol }{ }^{\circledR} \\
(0.25 \%) \\
\text { Topical formulation for } \\
\text { transdermal delivery } \\
\text { SLN based HG }\end{array}$ & $\begin{array}{l}\mathrm{LPV}^{\mathrm{c}} \\
\log \mathrm{P}=5.94\end{array}$ & 69.78 & N.D. & $-17.7 \pm 0.54$ & $48.86 \pm 4.6$ & $\begin{array}{l}\uparrow \text { sustained LPV release } \\
\text { from SLN based HG } \\
(71.197 \pm 0.006 \% \text { after } 12 \\
\text { h) compared to plain HG } \\
\text { of the drug released } \\
(98.406 \pm 0.007 \% \text { after } 4 \mathrm{~h}) \\
\text { SLN based HG resulted in } \\
\text { the highest } C_{\max }(20.3127 \\
\pm 6056 \mu \mathrm{g} / \mathrm{mL}) \text { compared } \\
\text { to plain HG }(8.0655 \pm \\
1.6369 \mu \mathrm{g} / \mathrm{mL}) \text { and oral } \\
\text { LPV suspension }(4.2550 \pm \\
16.380 \mu \mathrm{g} / \mathrm{mL})\end{array}$ & {$[72]^{*, * *, * * *}$} \\
\hline 2018 & $\begin{array}{l}\text { Lipid phase: hydrogenated } \\
\text { castor oil (castor wax) } \\
\text { Aqueous phase: sodium } \\
\text { oleate }(3.5 \%) \\
\text { Grafted SLN: peptide (100 } \\
\mu \mathrm{g}) \text { :SLN }(1 \mu \mathrm{M}) \\
\text { (Pept-DRV-SLN) }\end{array}$ & $\begin{array}{l}\mathrm{DRV}^{\mathrm{b}} \\
\log \mathrm{P}=1.89\end{array}$ & $\begin{array}{l}\text { DRV-SLN: } 90.10 \pm \\
1.15 \\
\text { Pept-DRV-SLN } \\
90.16 \pm 1.25\end{array}$ & $\begin{array}{l}\text { DRV-SLN: } \\
13.06 \pm \\
1.18 \\
\text { Pept-DRV- } \\
\text { SLN } \\
13.18 \pm \\
1.23\end{array}$ & $\begin{array}{l}\text { DRV-SLN: }-50.1 \\
\pm 1.17 \\
\text { Pept-DRV-SLN } \\
-35.45 \pm 1.10\end{array}$ & $\begin{array}{l}\text { D-SLN: } 189.45 \pm 2.10 \\
\text { Pept-D-SLN } \\
195.11 \pm 1.53\end{array}$ & $\begin{array}{l}\uparrow \text { DRV release in SLN } \\
\text { compared to a plain drug } \\
\text { suspension } \\
\uparrow \text { permeability in Caco-2 } \\
\text { cells (4-fold) than free } \\
\text { drug } \\
\uparrow \text { uptake in HIV host cells } \\
\text { (molt- } 4 \text { cells were taken as } \\
\text { a model containing CD4 } \\
\text { receptors) as compared to } \\
\text { non-CD4 receptor-bearing } \\
\text { Caco-2 cells } \\
\uparrow \text { bioavailability than free } \\
\text { DRV: } \\
\uparrow \text { uptake in various } \\
\text { organs (also in HIV } \\
\text { reservoirs like spleen and } \\
\text { brain) with } \\
\text { Pept-DRV-SLN } \\
\uparrow \text { binding with the HIV } \\
\text { host cells }\end{array}$ & {$[82]^{*, * *}$} \\
\hline
\end{tabular}


Table 4. Cont.

\begin{tabular}{|c|c|c|c|c|c|c|c|c|}
\hline \multirow[b]{2}{*}{ Year } & \multirow{2}{*}{ Composition } & \multirow[b]{2}{*}{ ARV } & \multicolumn{4}{|c|}{ Physicochemical Characterization } & \multirow[b]{2}{*}{ Outcomes } & \multirow[b]{2}{*}{ Ref. } \\
\hline & & & E.E. (\%) & D.L. (\%) & $\begin{array}{l}\zeta \text {-Potential } \\
(\mathrm{mV})\end{array}$ & Size $(\mathrm{nm})$ & & \\
\hline \multicolumn{9}{|l|}{ NLC } \\
\hline 2011 & $\begin{array}{l}\text { Lipid phase: OA }(1 \%) \text { and } \\
\text { Compritol }{ }^{\circledR} 888 \text { ATO/steric } \\
\text { acid }(4 \%) \\
\text { Aqueous phase: DODAB } \\
(1.8 \%), \text { Tween }{ }^{\circledR} 80(1 \%), \\
\text { lecithin }(0.2 \%) \text { and } \\
\text { 1-butanol }(0.5 \%) \\
\text { NLC coated with HSA }\end{array}$ & $\begin{array}{l}\mathrm{NVP} \\
\log \mathrm{P}=2.5\end{array}$ & $\begin{array}{l}\text { NVP-NLC: } \\
\approx 68 \text { (maximum } \\
\text { achieved) }\end{array}$ & N.D. & $\begin{array}{l}\text { NVP-NLC: }+ \\
\approx+17.5 \\
\uparrow[\text { HSA }] \downarrow \zeta \text { of } \\
\text { NLC to values } \\
\text { close to } \\
\text { neutrality }\end{array}$ & $\begin{array}{l}\text { NVP-NLC: } \\
159.6 \\
\text { HSA/NVP-NLC: N.D. }\end{array}$ & $\begin{array}{l}\text { NLC promote a fast } \\
\text { release }\end{array}$ & {$[120]^{* *}$} \\
\hline 2017 & $\begin{array}{l}\text { NLC } \\
\text { Lipid phase: Compritol }{ }^{\circledR} \\
\text { ATO 888:OA }(1: 3) \\
\text { Tween }{ }^{\circledR} 80(44 \mathrm{mg}) \\
\text { MLN } \\
\text { Lipid phase: } \text { Compritol }^{\circledR} \\
\text { ATO 888:OA:Span }{ }^{\circledR} 80 \\
(1: 3: 1.8) \\
\text { Tween }{ }^{\circledR} 80\end{array}$ & $\begin{array}{l}3 \mathrm{TC} \\
\log \mathrm{P}=-1.4\end{array}$ & $\begin{array}{l}\text { 3TC-NLC: } 34 \pm 1 \\
\text { 3TC-MLN: } 20 \pm 2\end{array}$ & $\begin{array}{l}\text { 3TC-NLC: } \\
0.3 \pm 0.01 \\
\text { 3TC-MLN: } \\
1.08 \pm \\
0.06\end{array}$ & $\begin{array}{l}\text { Unloaded-NLC: } \\
-42.9 \pm 0.7 \\
\text { 3TC-NLC: }-45 \\
\pm 2 \\
\text { Unloaded MLN: } \\
-24.5 \pm 0.4 \\
3 \text { TC-MLN: }-21 \\
\pm 2\end{array}$ & $\begin{array}{l}\text { Unloaded-NLC: } 229 \pm 2 \\
\text { 3TC-NLC: } 218 \pm 4 \\
\text { Unloaded MLN: } 426 \pm 9 \\
\text { 3TC-MLN: } 450 \pm 10\end{array}$ & $\begin{array}{l}\text { Sustained and controlled } \\
\text { 3TC release under gastric } \\
\text { and plasma-simulated } \\
\text { conditions }(\approx 45 \mathrm{~h} \text { in } \\
\text { MLN) } \\
\text { Low cytotoxicity ( } \mathrm{T} \\
\text { lymphocytes) for both } \\
\text { formulations } \\
\uparrow \text { loading capacity and } \\
\text { storage stability with } \\
\text { MLN }\end{array}$ & {$[123]^{* *}$} \\
\hline 2018 & $\begin{array}{l}\text { Lipid phase: } \\
\text { Precirol }{ }^{\circledR} \text { ATO 5:Miglyol }{ }^{\circledR} \\
812(3: 1) \\
\text { Tween }{ }^{\circledR} 80(158 \mathrm{mg}) \\
\text { AZT-NLC prepared by hot } \\
\text { ultrasonication method and } \\
\text { M-AZT-NLC prepared by } \\
\text { the one-step } \\
\text { microwave-assisted method }\end{array}$ & $\begin{array}{l}\mathrm{AZT} \\
\log \mathrm{P}=0.05\end{array}$ & $\begin{array}{l}\text { AZT-NLC: } 44 \pm 3 \\
\text { M-AZT-NLC: } 22 \pm \\
2\end{array}$ & $\begin{array}{l}\text { AZT-NLC: } \\
0.31 \pm \\
0.04 \\
\text { M-AZT- } \\
\text { NLC: } 1.41 \\
\pm \\
0.02\end{array}$ & $\begin{array}{l}- \\
\text { AZT-NLC: }-29 \\
\pm 2 \\
\text { M-AZT-NLC: } \\
-20 \pm 1\end{array}$ & $\begin{array}{l}\text { AZT-NLC: } 266 \pm 4 \\
\text { M-AZT-NLC: } 113 \pm 3\end{array}$ & $\begin{array}{l}\text { Controlled release of AZT } \\
\text { Suitable for oral } \\
\text { administration }\end{array}$ & {$[12]^{* *}$} \\
\hline
\end{tabular}


Table 4. Cont.

\begin{tabular}{|c|c|c|c|c|c|c|c|c|}
\hline \multirow[b]{2}{*}{ Year } & \multirow[b]{2}{*}{ Composition } & \multirow[b]{2}{*}{ ARV } & \multicolumn{4}{|c|}{ Physicochemical Characterization } & \multirow[b]{2}{*}{ Outcomes } & \multirow[b]{2}{*}{ Ref. } \\
\hline & & & E.E. $(\%)$ & D.L. (\%) & $\begin{array}{l}\text { c-Potential } \\
(\mathrm{mV})\end{array}$ & Size (nm) & & \\
\hline 2019 & $\begin{array}{l}\text { Lipid phase: Compritol }{ }^{\circledR} \\
888 \text { ATO:OA }(3.7: 1) \\
\text { Aqueous phase: Tween }{ }^{\circledR} \\
80(0.04)\end{array}$ & $\begin{array}{l}\mathrm{LPV}^{\mathrm{b}} \\
\log \mathrm{P}=5.94\end{array}$ & 83.6 & N.D. & +21.2 & 196.6 & $\begin{array}{l}\uparrow \text { bioavailability } \\
\uparrow[\mathrm{LPV}] \text { in the brain } \\
\uparrow \text { uptake and } \downarrow \\
\text { cytotoxicity (Caco-2 cells } \\
\text { and macrophages) }\end{array}$ & {$[83]^{*, * *}$} \\
\hline 2020 & $\begin{array}{l}\text { Lipid phase: } \text { Precirol }^{\circledR} \text { ATO } \\
\text { 5:Lauroglycol } \\
\text { 90 (70:30) } \\
\text { Cremophor }^{\circledR} \text { RH } 40(3 \%)\end{array}$ & $\begin{array}{l}\mathrm{ATV} \mathrm{b}^{\mathrm{b}} \\
\log \mathrm{P}=4.5\end{array}$ & $71.09 \pm 5.84$ & $8.12 \pm 2.7$ & $-11.7 \pm 0.47$ & $227.6 \pm 5.4$ & $\begin{array}{l}\text { Fast release }(60 \%) \text { in the } \\
\text { initial } 2 \mathrm{~h} \text {, followed by } \\
\text { sustained release } \\
\uparrow \text { permeation of ATV } \\
(2.36 \text {-fold) across the rat } \\
\text { intestine as compared to } \\
\text { the free drug } \\
2.75 \text {-fold greater } \mathrm{C}_{\max } \text { in } \\
\text { the brain and a } 4 \text {-fold } \\
\text { improvement in brain } \\
\text { bioavailability as } \\
\text { compared to the free drug }\end{array}$ & {$[86]^{*, * *, * * *}$} \\
\hline 2020 & $\begin{array}{l}\text { Lipid phase: } \text { Monosteol }{ }^{\mathrm{TM}} \\
(71.5 \%) \text { : Capmul }{ }^{\circledR} \text { PG } 8 \\
(28.5 \%) \\
\text { Aqueous phase: } \\
\text { Tween }{ }^{\circledR} 80(0.43 \%) \text { and } \\
\text { poloxamer } 188(1.3 \%) \\
\text { Cationic NLC } \\
\text { Lipid phase: same } \\
\text { composition } \\
\text { Aqueous phase: same } \\
\text { composition + CTAB }(1 \% \\
\text { w/w of lipid phase })\end{array}$ & $\begin{array}{l}\mathrm{EFV} \\
\log \mathrm{P}=4.6\end{array}$ & $\begin{array}{l}\text { EFV-NLC: } 91.18 \pm \\
2.9 \\
\text { Cationic EFV-NLC: } \\
90.21 \pm 2.3\end{array}$ & $\begin{array}{l}\text { EFV-NLC: } \\
10.94 \pm \\
0.35 \\
\text { Cationic } \\
\text { EFV-NLC: } \\
11.04 \pm \\
0.17\end{array}$ & $\begin{array}{l}\text { Plain NLC: } \\
-15.16 \pm 0.69 \\
\text { EFV-NLC: }-15.8 \\
\pm 1.21 \\
\text { Cationic } \\
\text { EFV-NLC: } \\
+23.86 \pm 0.49\end{array}$ & $\begin{array}{l}\text { Plain NLC: } 114.53 \pm \\
5.63 \\
\text { EFV-NLC: } 116.5 \pm 9.59 \\
\text { Cationic EFV-NLC: } \\
105.6 \pm 4.93\end{array}$ & $\begin{array}{l}\uparrow \text { solubility } \\
\text { Excellent } \\
\text { cytocompatibility }\left(\mathrm{CC}_{50}\right. \\
13.23 \pm 0.54 \mu \mathrm{g} / \mathrm{mL}) \\
\text { Uptake of cationic NLC } \\
\text { by THP- } 1 \text { macrophages } \\
\uparrow \text { retention/sustained } \\
\text { release and } \uparrow \text { inhibition of } \\
\text { HIV-1 (2.32-fold) in } \\
\text { infected macrophages } \\
\text { with cationic NLC } \\
\text { compared to the free drug } \\
\uparrow \text { anti-HIV-efficacy } \\
\text { (2.29-fold) with cationic } \\
\text { NLC }\end{array}$ & {$[124]^{* *}$} \\
\hline
\end{tabular}


Table 4. Cont.

\begin{tabular}{|c|c|c|c|c|c|c|c|c|}
\hline \multirow[b]{2}{*}{ Year } & \multirow{2}{*}{ Composition } & \multirow[b]{2}{*}{ ARV } & \multicolumn{4}{|c|}{ Physicochemical Characterization } & \multirow[b]{2}{*}{ Outcomes } & \multirow[b]{2}{*}{ Ref. } \\
\hline & & & E.E. $(\%)$ & D.L. (\%) & $\begin{array}{l}\text { c-Potential } \\
(\mathrm{mV})\end{array}$ & Size (nm) & & \\
\hline 2021 & $\begin{array}{l}\text { Lipid phase: } \\
{\text { Precirol }{ }^{\circledR} \text { ATO } 5: \text { Capmul }^{\circledR}} \\
\text { MCM }(40: 60) \\
\text { Capryol }^{\mathrm{TM}} 90 \text { (N.D.) } \\
\text { Aqueous phase: Lutrol }{ }^{\circledR} \mathrm{F} \\
127(1 \%)\end{array}$ & $\begin{array}{l}\text { ETR }^{\mathrm{a}} \\
\log \mathrm{P}=4.5\end{array}$ & $>90$ & 5 to 10 & $-20 \pm 2.3$ & $351.7 \pm 3.36$ & $\begin{array}{l}\uparrow \text { cellular uptake and } \uparrow \\
\text { anti-HIV efficacy } \\
\text { Overall better } \\
\text { pharmacokinetics as } \\
\text { compared to the free drug } \\
\uparrow[\text { [ETR] several-fold in the } \\
\text { liver, ovary, lymph node, } \\
\text { and brain as compared to } \\
\text { the free drug }\end{array}$ & {$[87]^{*, * *}$} \\
\hline 2021 & $\begin{array}{l}\text { Lipid phase: } \\
\text { GMS/Gelucire }^{\circledR} \\
\text { 50/13/Dynasan } \\
\text { 118:Capmul }{ }^{\circledR} \text { MCM EP } \\
(80: 20)(8 \%) \\
\text { Span }{ }^{\circledR} 80 \\
\text { Aqueous phase: } \\
\text { Tween }{ }^{\circledR} 80, \text { sodium cholate; } \\
\text { PEG } 6000(1 \%) \text {, propylene } \\
\text { glycol }(1 \%), \text { BHT }(0.4 \%) \\
\text { Note: Surfactant mixture } \\
\text { [(Tween }{ }^{\circledR} 80: \text { Span }^{\circledR} 80 \\
(70: 30)]: 5 \%\end{array}$ & $\begin{array}{l}\text { DTG } \\
\log P=2.2\end{array}$ & 88.09 & N.D. & -16.6 & 123.1 & $\begin{array}{l}\text { Sustained release over } 48 \\
\mathrm{~h} \\
\uparrow \text { DTG permeation } \\
\text { through rat intestine } \\
(\approx 94.02 \%) \text { as compared to } \\
\text { plain drug suspension } \\
\text { (only } 55.62 \%) \text { after } 8 \mathrm{~h}\end{array}$ & {$[125]^{* * * * * *}$} \\
\hline 2014 & $\begin{array}{l}\text { Lipid phase: Precirol }{ }^{\circledR} \text { ATO } \\
15(10 \%) \text { and Miglyol }{ }^{\circledR} 812 \\
(1 \%) \\
\text { Aqueous phase: Tween }{ }^{\circledR} \\
80(1 \%) \text { and poloxamer } 188 \\
(1 \text { or } 0.5 \%) \\
\text { Coating on NLC: Dex-Prot }\end{array}$ & $\begin{array}{l}\mathrm{SQV} \\
\log \mathrm{P}=3.8\end{array}$ & $\begin{array}{l}\text { All formulations: } \\
99\end{array}$ & N.D. & $\begin{array}{l}\text { Uncoated NLC: } \\
-36 \pm 6 \text { to }-22 \\
\pm 4 \\
\text { Dex-Prot NLC: } \\
-0.5 \pm 4 \text { to }+12 \\
\pm 4\end{array}$ & $\begin{array}{l}\text { Uncoated NLC: } 152 \pm 1 \\
\text { to } 936 \pm 1 \\
\text { Dex-Prot NLC: } 244 \pm 1 \\
\text { to } 1326 \pm 1\end{array}$ & $\begin{array}{l}\uparrow \text { permeability (up to } \\
\text { 9-fold) with Dex-Prot } \\
\text { NLC in comparison to } \\
\text { uncoated NLC } \\
\text { (Caco-2/HT29-MTX } \\
\text { co-culture monolayer } \\
\text { model) }\end{array}$ & {$[126]^{* *}$} \\
\hline
\end{tabular}


Table 4. Cont.

\begin{tabular}{|c|c|c|c|c|c|c|c|c|}
\hline \multirow[b]{2}{*}{ Year } & \multirow{2}{*}{ Composition } & \multirow{2}{*}{ ARV } & \multicolumn{4}{|c|}{ Physicochemical Characterization } & \multirow{2}{*}{ Outcomes } & \multirow[b]{2}{*}{ Ref. } \\
\hline & & & E.E. $(\%)$ & D.L. (\%) & $\begin{array}{l}\zeta \text {-Potential } \\
(\mathrm{mV})\end{array}$ & Size $(\mathrm{nm})$ & & \\
\hline 2017 & $\begin{array}{l}\text { Lipid phase: Precirol }{ }^{\circledR} \text { ATO } \\
\text { 5:Captex }{ }^{\circledR} \text { P } 500(7: 3) \\
\text { Aqueous phase: } \\
\text { MYS-25 }(2 \%)\end{array}$ & $\begin{array}{l}E F V ~ a, d \\
\log P=4.6\end{array}$ & $95.78 \pm 0.42$ & N.D. & $-18.7 \pm 1.0$ & $161 \pm 2.8$ & $\begin{array}{l}\text { EFV release of } 92.45 \% \\
\text { after } 24 \mathrm{~h} \\
\text { The therapeutic } \\
\text { concentration of EFV in } \\
\text { the CNS following } \\
\text { intranasal administration } \\
\text { No toxicity of } \\
\text { encapsulated EFV } \\
\text { compared to free EFV }\end{array}$ & {$[54]^{*, * *}$} \\
\hline
\end{tabular}

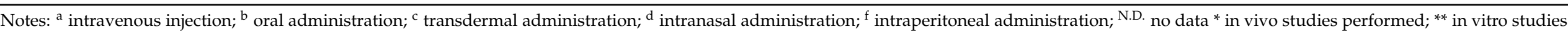

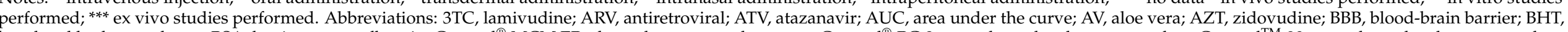

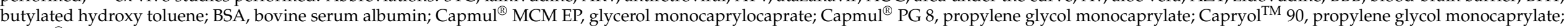

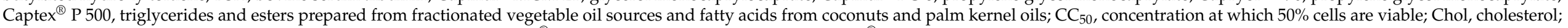

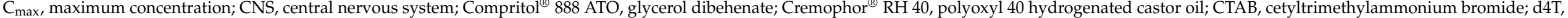

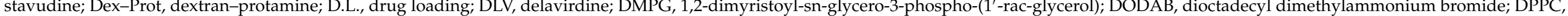

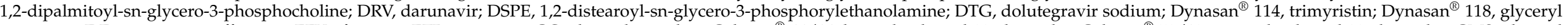

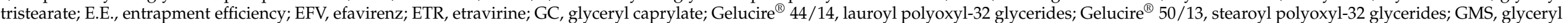

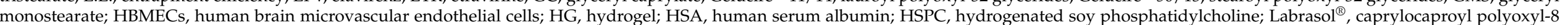

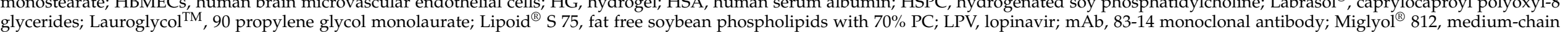

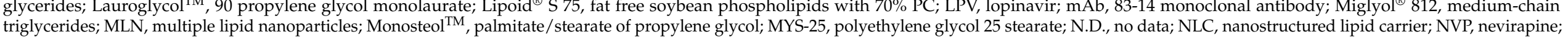

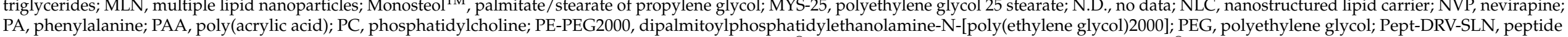

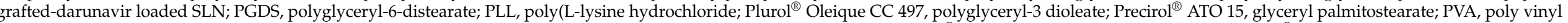

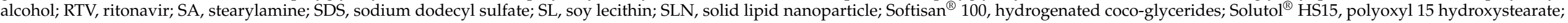
$\mathrm{QV}$, saquinavir; TFV, tenofovir; w/w- weight/weight; wt-weight. 
Table 5. Lipid emulsions (microemulsions, nanoemulsions, and self-emulsifying drug delivery systems) for ARV delivery.

\begin{tabular}{|c|c|c|c|c|c|c|c|c|}
\hline \multirow{2}{*}{ Year } & \multirow{2}{*}{ Composition } & \multirow{2}{*}{ ARV } & \multicolumn{4}{|c|}{ Physicochemical Characterization } & \multirow{2}{*}{ Outcomes } & \multirow{2}{*}{ Ref. } \\
\hline & & & E.E. (\%) & D.L. (\%) & $\zeta$-Potential (mV) & Size (nm) & & \\
\hline \multicolumn{9}{|c|}{ Microemulsions (ME) } \\
\hline 2016 & $\begin{array}{l}\text { Lipid phase: isopropyl } \\
\text { myristate }(10 \%), \text { Labrasol }^{\circledR} \\
(30 \%) \text {; Oleic Plurol }{ }^{\circledR}(10 \%)\end{array}$ & $\begin{array}{l}\mathrm{AZT} \mathrm{T}^{\mathrm{c}} \\
\log \mathrm{P}=0.05\end{array}$ & N.D. & N.D. & N.D. & N.D. & $\begin{array}{l}\uparrow \text { AZT permeated }(\approx 2 \text {-fold) as } \\
\text { compared to control-HGNo } \\
\text { apparent skin irritation; little } \\
\text { histological changes in mice skin }\end{array}$ & {$[128]^{*, * *}$} \\
\hline \multicolumn{9}{|c|}{ Nanoemulsions (NE) } \\
\hline 2008 & $\begin{array}{l}\text { o/w } \\
\text { Lipid phase: Flax-seed oil } \\
\text { or safflower oil }(1 \mathrm{~mL}) \\
\text { Aqueous phase: } \mathrm{EPC}(3 \%) \\
\text { and deoxycholic acid }(1 \%)\end{array}$ & $\begin{array}{l}\mathrm{SQV}^{\mathrm{a}, \mathrm{b}} \\
\log \mathrm{P}=3.8\end{array}$ & N.D. & N.D. & $\begin{array}{l}\text { SQV-Flax-seed NE: } \\
-43.28 \pm 3.79 \\
\text { SQV-Safflower NE: } \\
-49.55 \pm 5.02\end{array}$ & $\begin{array}{l}\text { SQV-Flax-seed } \\
\text { NE:218.0 } \pm \\
\text { 13.9SQV-Safflower } \\
\text { NE:140.0 } \pm 12.6\end{array}$ & $\begin{array}{l}\uparrow \mathrm{SQV} \text { (3-fold) in systemic } \\
\text { circulation when loaded in } \\
\text { Flax-seed NE than in the free } \\
\text { form } \uparrow \text { bioavailability } \uparrow \text { brain } \\
\text { distribution }\left(C_{\max } 5 \text {-fold and }\right. \\
\text { AUC } 3 \text {-fold) higher in the brain } \\
\text { with Flax-seed NE than the free } \\
\text { drug }\end{array}$ & {$[90] *$} \\
\hline
\end{tabular}


Table 5. Cont.

\begin{tabular}{|c|c|c|c|c|c|c|c|c|}
\hline \multirow{2}{*}{ Year } & \multirow{2}{*}{ Composition } & \multirow{2}{*}{ ARV } & \multicolumn{4}{|c|}{ Physicochemical Characterization } & \multirow{2}{*}{ Outcomes } & \multirow{2}{*}{ Ref. } \\
\hline & & & E.E. (\%) & D.L. (\%) & $\zeta$-Potential (mV) & Size $(\mathrm{nm})$ & & \\
\hline 2014 & $\begin{array}{l}\text { NE } \\
\text { Lipid phase: Capryol }{ }^{\circledR} 90, \\
\text { Geucire }^{\circledR} 44 / 14(13.728 \%) \\
\text { Aqueous phase: } \\
\text { Transcutol }{ }^{\circledR} \mathrm{HP}(3.432 \%) \\
\text { and water }(79.98 \%) \\
\text { Oil:Smix (1:6) }\end{array}$ & $\begin{array}{l}\mathrm{EFV}^{\mathrm{b}} \\
\log \mathrm{P}=4.6\end{array}$ & N.D. & N.D. & N.D. & $26.427 \pm 1.960$ & $\begin{array}{l}>80 \% \text { release within } 6 \mathrm{~h} \uparrow \\
\mathrm{AUC}_{0 \rightarrow 24 \mathrm{~h}}(43.53 \mu \mathrm{g} \mathrm{h} / \mathrm{mL}) \\
\text { compared to EFV suspension } \\
(20.65 \mu \mathrm{g} \mathrm{h} / \mathrm{mL}) \mathrm{EFV} \text { absorption } \\
\text { resulted in } 2.6 \text {-fold increase in } \\
\text { bioavailability in comparison to } \\
\text { the free EFV }\end{array}$ & {$[129]^{* * * *}$} \\
\hline 2014 & $\begin{array}{l}\text { o/w NE } \\
\text { Lipid phase: Capmul } \\
\text { MCM (6\%) } \\
\text { Aqueous phase: Tween }{ }^{\circledR} \\
80(6 \%) \text {, PEG } 400(2 \%) \text { and } \\
\text { water }(86 \%)\end{array}$ & $\begin{array}{l}\text { SQVM } \\
\log P=3.8\end{array}$ & $96.8 \pm 1.2$ & N.D. & $-10.3 \pm 1.67$ & $176.3 \pm 4.21$ & $\begin{array}{l}\uparrow \text { Diffusion of SQVM across nasal } \\
\text { mucosa than the free drug No } \\
\text { significant adverse effect in cilia } \\
\text { toxicity study } \uparrow[S Q V M] \text { brain } \\
\text { after intranasal administration of } \\
\text { NE than intravenous delivery of } \\
\text { free drugEffective CNS targeting }\end{array}$ & {$[88]^{*, * *}$} \\
\hline 2013 & $\begin{array}{l}\text { o/w NE } \\
\text { Lipid phase: soya bean oil } \\
(10 \%), \text { Chol }(0 \text { or } 0.3 \%), \\
\text { EPC-80 }(1.2 \%) \text {, } \\
\alpha \text {-tocopherol }(0.25 \%) \text { OA } \\
(0.3 \%) \\
\text { Aqueous phase: glycerol } \\
(2.25 \%), \text { Tween }{ }^{\circledR} 80(0 \text { to } \\
1 \%) \text {, and double-distilled } \\
\text { water }(10 \%)\end{array}$ & $\mathrm{IDV}^{\mathrm{a}}$ & $\begin{array}{l}\text { No Chol, no } \\
\text { Tween }{ }^{\circledR} \\
80: \\
99.1 \pm 0.2 \\
\text { Chol and no } \\
\text { Tween }{ }^{\circledR} 80: \\
98.9 \pm 0.03 \\
\text { Tween }{ }^{\circledR} 80 \\
(1 \%): \\
98.97 \pm 0.2\end{array}$ & N.D. & $\begin{array}{l}\text { No Chol and no } \\
\text { Tween } 80 \mathrm{NE:} \\
-35.8 \pm 6.04 \\
\text { Chol and no } \\
\text { Tween } 80 \mathrm{NE}: \\
-31.3 \pm 1.80 \\
\text { Tween } 80(1 \%) \mathrm{NE} \text { : } \\
-40.1 \pm 4.05\end{array}$ & $\begin{array}{l}\text { No Chol and no } \\
\text { Tween } 80 \text { NE:329.5 } \\
\pm 3.08 \text { Chol and } \\
\text { no Tween } 80 \\
\text { NE:237.0 } \pm 5.08 \\
\text { Tween } 80(1 \%) \\
\text { NE:196.0 } \pm 3.54\end{array}$ & $\begin{array}{l}\text { NE containing Chol and higher } \\
\text { [Tween } 80 \text { ] (1\%) had lower } \\
\text { globule size, relative better } \\
\text { release, and higher } \zeta \uparrow \text { brain } \\
\text { uptake of IDV in Tween } 80 \text { (1\%) } \\
\text { NE compared to Chol and no } \\
\text { Tween } 80 \text { NE } \uparrow \text { brain level of IDV } \\
\text { administered by Tween } 80(1 \%) \\
\text { NE compared to the free drug } \\
\text { (2.44-fold) } \uparrow \text { IDV brain-specific } \\
\text { accumulation }\end{array}$ & {$[89]^{*, * *}$} \\
\hline
\end{tabular}


Table 5. Cont.

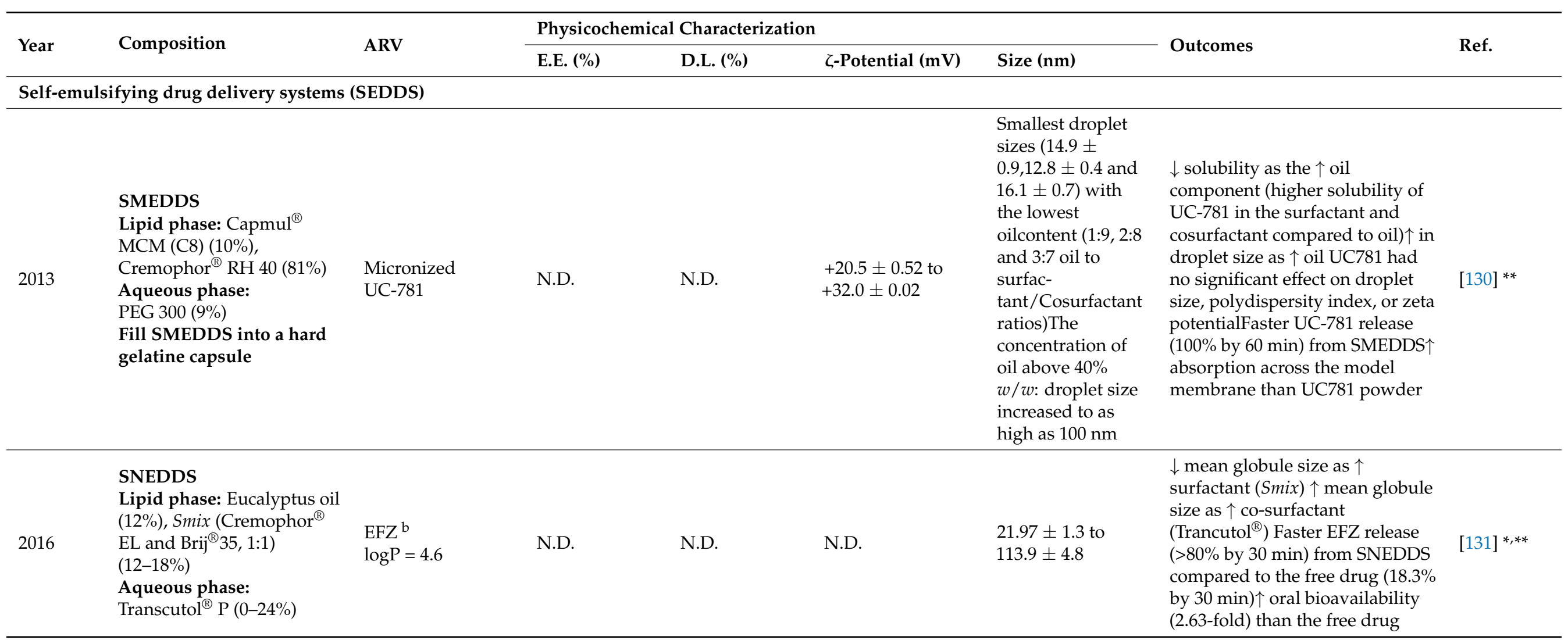


Table 5. Cont.

\begin{tabular}{|c|c|c|c|c|c|c|c|c|}
\hline \multirow{2}{*}{ Year } & \multirow{2}{*}{ Composition } & \multirow{2}{*}{ ARV } & \multicolumn{4}{|c|}{ Physicochemical Characterization } & \multirow{2}{*}{ Outcomes } & \multirow{2}{*}{ Ref. } \\
\hline & & & E.E. $(\%)$ & D.L. $(\%)$ & $\zeta$-Potential (mV) & Size $(\mathrm{nm})$ & & \\
\hline 2016 & 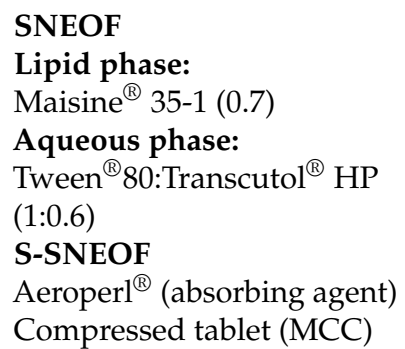 & $\begin{array}{l}\mathrm{LPV}^{\mathrm{b}} \\
\log \mathrm{P}=5.94\end{array}$ & $99.45 \pm 0.59$ & N.D. & N.D. & $\begin{array}{l}\text { SNEOF: } \\
\text { 53.16SNEOF } \\
\text { tablets: } 80\end{array}$ & $\begin{array}{l}\uparrow \text { LPV release }(60 \% \text { by } 10 \mathrm{~min}) \\
\text { Lymphatic uptake of LPV from } \\
\text { SNEOF } \uparrow \text { rate and extent of oral } \\
\text { bioavailability than the free drug }\end{array}$ & {$[66]^{*, * *}$} \\
\hline
\end{tabular}

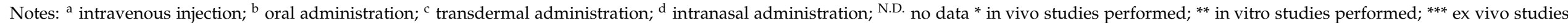

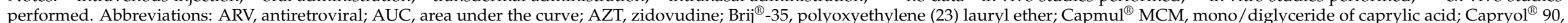

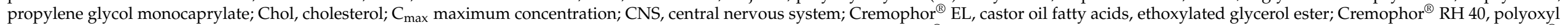

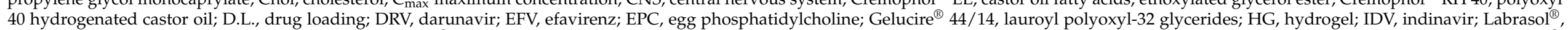

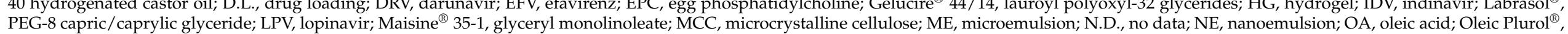

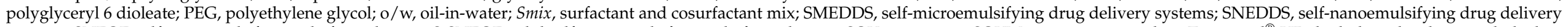

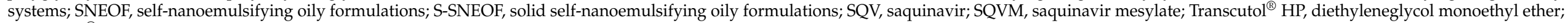
Transcutol ${ }^{\circledR} \mathrm{P}$, diethylene glycol monoethyl ether. 
Subsequently, a detailed and critical analysis of studies selected from Tables 3-5 is presented in Section 3.1 with a focus on the route of administration and Section 3.2 that focused on targeting strategies.

\subsection{Tuning the Physicochemical Properties of Lipid-Based Nanocarriers to Overcome Biological Barriers}

According to the administration route (Figure 7) and to achieve particularly in vivo performance and clinical applications, specific aspects of nanocarriers such as composition, size, surface charge, and coating need to be tuned [132-136].

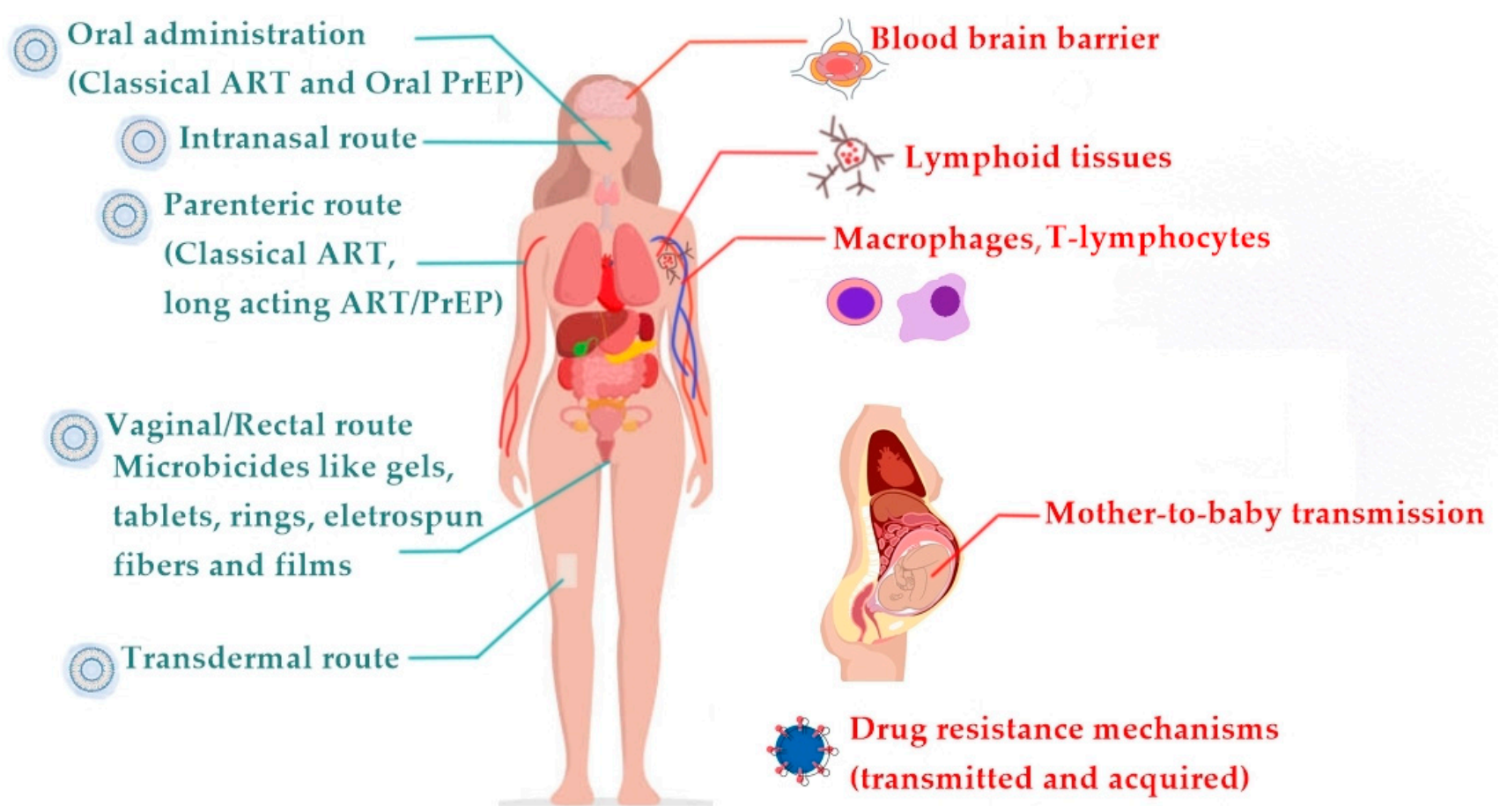

Figure 7. Potential ART (antiretroviral therapy) administration routes (left side in blue). The nanocarrier cartoon marks all possible routes of administration for lipid-based nanosystems. Classical ART formulations for therapy or pre-exposure prophylaxis (PrEP) are limited to oral and parenteral routes. Biological barriers to ART administration (right side in red).

Several studies have been conducted for parenteral administration of ARV (e.g., subcutaneous, intravenous). In this case, it is critical to extend the circulatory residence of the nanocarriers to ensure adequate time for distribution to the target tissues. Avoiding opsonization of serum proteins (e.g., human serum albumin, HSA) by controlling the size $(<250 \mathrm{~nm})$, charge (avoid positively charged nanocarriers), and surface coating with hydrophilic polymers (e.g., polyethylene glycol, PEG) are some strategies for extending circulation time. These criteria were met by Gagné et al. and Sudhakar et al. (liposomes) [80,94], by Heiati et al. (SLN) [18] and Pokharkar et al. (NLC) [54]. Aside from extending the circulation time of nanocarriers, it is also critical to use targeting strategies that can deliver ARV drugs to sites of latent HIV reservoirs such as lymph nodes, the spleen, and the gut mucosa, where HIV-target cells such as memory CD4+ T cells, macrophages, microglia, and astrocytes in the CNS are prevalent [137]. Some of these targeting strategies include: (i) surface functionalization of nanocarriers with sugar molecules like mannose $[13,99]$ or galactose $[14,15,96]$ that are recognized by lectin receptors found on the surface of cells from the mononuclear phagocyte system (MPS); (ii) coating of nanocarriers with hydrophilic molecules (e.g., amino acids, glucose) to facilitate BBB permeation by carrier-mediated transcytosis [55]; (iii) engineering of the lipid matrix of the nanocarriers (SLN, NLC, nanoemulsions) in order to mimic low-density lipoproteins (LDL) that are recognized by LDL receptors, thus facilitating BBB permeation by receptor-mediated 
transcytosis [54,55,64,65,85,87,89,90,120]; (iv) functionalization with ligands (e.g., HSA and monoclonal antibody $(\mathrm{mAb}))$ that enhance BBB permeation by receptor-mediated transcytosis [64,120]; (v) inhibition of P-gp, which increases brain-specific accumulation [89]; and (vi) magnetic aided transport across BBB [115] and to MPS cells [116].

Oral administration is one of the preferred routes of administration due to its convenience that assures better adherence to the therapeutic regimens. However, this route of administration presents several limitations such as the variable absorption of the drugs, drug degradation by enzymes and acidic $\mathrm{pH}$ in the stomach, and first-pass metabolism effect. The physicochemical properties of drugs determine their absorption through the GI tract, namely their lipophilicity, which can be assessed by the logP. Typically, only drugs with $\log \mathrm{P}$ values between 1 and 3 have favorable oral absorption profiles [138]. Most ARV drugs are outside this range (Tables 3-5), being either extremely hydrophilic (e.g., ddI [97] and AZT [121]) or highly lipophilic (e.g., LPV [66,71,83,109], RTV [110], EFV $[16,75,129]$, SQV [90,126], EFZ [131] and ATV [86]). Therefore, lipid-based nanocarriers may help ARV drugs achieving a balanced lipophilic/hydrophilic nature. Additionally, lipid-based nanocarriers can be site-specific delivery systems by modifying their surface with ligands that are recognized at target tissues. For example, following oral administration, biotinylated liposomes of insulin were observed to permeate the GI tract via a facilitated absorption mechanism [139]. Based on this study, liposomes were coated with biotin (biotinylated proliposomes) to improve uptake of RTV into the intestinal lymphatic tissues [110]. Another example is the SLN grafting with a peptide that is specific for CD4+ receptors present on T cells, which improved specific DRV uptake by HIV host cells [82].

The nature of the components of nanocarriers also influences their functional performance when administered via a specific route. In the case of the oral route, the components of lipid-based nanocarriers induce the production of endogenous biliary lipids, which form colloidal structures in the presence of bile salts and significantly improve the solubilization and absorption capacity of ARVs in the small intestine [51]. Furthermore, the inclusion of penetration enhancers (e.g., Transcutol ${ }^{\circledR}[66,127,129,131]$ and biliary salts (deoxycholic acid, sodium cholate) $[90,125]$ ) in the lipid matrix composition also improves the oral delivery of ARVs agents.

Transdermal administration, as opposed to oral administration, avoids the first-pass metabolism effect of drugs. As a result, a lower quantity of drugs can be administered efficiently by the transdermal route with reduced toxicity to achieve the same bioavailability as the oral route $[72,113]$. The fact that not all drugs can be delivered transdermally is one of the major drawbacks of this method. Drugs with a high molecular weight ( $>500 \mathrm{Da}$ ) cannot penetrate the stratum corneum [11]. In the pharmaceutical field, lipidbased nanocarriers are the most used for dermal/transdermal drug delivery. To improve skin permeation and efficiency, the composition of liposomes is changed to create new classes of lipid vesicles known as transferosomes, niosomes, ethosomes, cubosomes, and tocosomes. Jain et al. developed ethosomes that, due to the high amounts of ethanol, aid in breaking the stratum corneum and have higher elasticity, which contributes to improved 3TC skin permeation [112]. Chettupalli et al. produced cubosomes that improved ATV transdermal permeation due to the bioadhesive and permeation enhancer effect of their components [113]. SLN and nanoemulsions have also proved effective for the transdermal delivery of LPV [72] and AZT [128] respectively.

The vaginal administration is a promising route that allows self-administration of ARV drugs and permits achieving both local and systemic effects. In the case of local administration, the vaginal route avoids systemic exposure reducing side effects. If systemic administration is intended then drugs should have hydrophobic properties and low molecular weight $[140,141]$. The vaginal route may also be advantageous for drugs that undergo extensive metabolism, as it avoids the hepatic first-pass effect and allows for a reduction in the doses of drugs administered [141]. However, the vaginal route has been exclusively considered for topical pre-exposure prophylaxis (PrEP), as a preventative approach. Due to the unique characteristics of this mucosal site, administering ARV drugs via the vaginal 
route is a huge challenge because a fine-tuning of mucoadhesiveness/muco-penetration is required to ensure good distribution along the cervicovaginal lumen. In this regard, lipid nanocarriers can be used to improve ARV permeation into the vaginal mucosa, but there are some requirements in terms of size ( $>100$, preferentially $200-500 \mathrm{~nm})$ and surface charge (positively charged nanocarriers are mucoadhesive and hinder diffusion, whereas PEGylation promotes mucosal permeation) [142]. These requirements were considered in an in vitro study in which liposomal hydrogels were developed for the delivery of two ARV drugs with different lipophilicities [52]. As such, the hydrogel (hydrophilic) was used as a carrier for the hydrophilic drug FTC, while the liposomes were used as carriers for the more lipophilic drug TDF. The size and zwitterionic charge of the liposomes, as well as the hydrophilic nature of the gel, imply that there are fewer interactions with mucin from the mucosa, which may translate to higher drug diffusion [52]. SLN was also strategically developed to improve TFV uptake by virus-infected cells via vaginal administration [118]. TFV-loaded SLN were functionalized with a combination of peptide (PLL), to enhance intracellular uptake of the drug, and heparin, which can direct nanocarriers to killer lectin-like receptors of natural killer (NK) cells, resulting in direct killing of virus-infected cells [118]. Moreover, SLN possessed an adequate size and high density of negative surface charge that creates a hydrophilic surface that facilitates diffusion and minimizes entrapment into mucus [118]. In another study, a hybrid system composed of polymeric nanofibers containing liposomes loaded with FTC and TDF provided rapid onset of local ARV levels in mice after a single vaginal administration compared to five days of continuous daily use of oral TDF/FTC [117]. These results may be also translatable into a fairly wide protection time window in humans [117].

Intranasal administration has recently been investigated as a potential alternative to intravenous and other systemic administration routes for providing direct access to the brain via axonal transport along the olfactory nerve [50]. This administration route has the advantage of increased bioavailability due to the absence of first-pass liver metabolism and subsequent rapid absorption, resulting in a rapid therapeutic effect [50]. The disadvantages of this route are related to the limited amounts of drugs that can be delivered into the brain and to the mucociliary clearance mechanism that can remove toxic substances, drugs, nanocarriers, and microorganisms caught in the mucus layer [50]. To overcome the mucociliary clearance mechanism, the lipid matrix composition, and the surface chemistry of the nanocarriers have been explored for ARV delivery. Tuning the surface coating is important to guarantee enough mucoadhesion to avoid the rapid removal of lipid nanocarriers from the nasal mucosa [50]. On the other hand, it is also necessary to impart the nanocarrier surface with mucopenetrating properties to improve diffusion from the nose to the brain [50]. For example, Pokharkar et al. and Mahajan et al. used PEG coatings as amucopenetrating strategy for intranasal brain delivery of EFV [54] and SQVM [88], respectively. Other ARV drugs (SQV and EFV) benefited from nanocarriers composed of lipids with mucoadhesive properties (e.g., monoolein) [114] or fatty acids with mucopenetration properties [54,84]. The ability of nanocarriers composed of fatty acids to be flexible and pass through the opening of the olfactory epithelium has been attributed to the surfactant nature of fatty acids, which may disrupt the nasal membrane [50].

\subsection{Targeting Anatomical and Cellular Reservoirs}

As previously mentioned, lipid nanocarriers' surfaces can be functionalized to improve their targeting selectivity [143]. The reticuloendothelial system contains galactose and lectin receptors and thus galactosylated $[14,15,96]$ and mannosylated $[13,99]$ liposomes target these receptors and have been utilized to deliver AZT, ddI, and d4T to the reticuloendothelial system. Functionalization of lipid nanocarriers with $\mathrm{mAb}$, such as anti-HLA-DR that target follicular dendritic cells, B cells, and macrophages that express the HLA-DR is another strategy to achieve targeting specificity. For example, immunoliposomes functionalized with $\mathrm{mAb}$ resulted in increased IDV accumulation in mouse lymph nodes, with an area-under-the-curve that was 126-fold more than that of the free drug [94]. Liposomes 
can also be coated with recombinant soluble CD4 molecules [144,145], the Fab' fragment of monoclonal antibody F105 [100], or the Fab' fragment of anti-HLA-DR antibody [146], which all target gp120 on HIV-infected cells $[143,145]$. Besides sugars and $\mathrm{mAb}$, PEG is also a surface targeting moiety that increases lipid nanocarriers at the lymph nodes $[143,147]$.

In the case of BBB targeting the lipid-based nanocarriers have the potential to reduce efflux transporter binding by increasing brain accumulation. When ATV was encapsulated in solid lipid nanoparticles, its accumulation in a human brain microvessel endothelial cell line (hCMEC/D3) was greatly increased, indicating that this is a promising strategy for delivering ATV across the BBB [65]. When SQV was given by oil-in-water nanoemulsions synthesized with essential polyunsaturated fatty acid-rich oils, the maximal concentration and area-under-the-curve values in the brain were five- and threefold higher than the aqueous suspension [90]. Besides the lipid nanocarrier composition, it is advisable their surface functionalization with targeting ligands (e.g., Transferrin, and apolipoproteins) [47] that are recognized by BBB receptors and favor BBB transcytosis (examples of additional targeting ligands for BBB crossing can be consulted in [56]). Finally, one of the most tested cell-penetrating peptides is the HIV-1 Tat peptide. Certain sections of this peptide, known as protein-transduction domains, can help it migrate through biological membranes. The fusing of -galactosidase to the Tat peptide is required for BBB permeability, which is independent of transporters and receptor-mediated endocytosis [47]. Hence surface functionalization of lipid nanocarriers with Tat peptide can be an effective strategy for BBB crossing [47]. Glutathione is another peptide that is frequently utilized to achieve brain targeting. This endogenous tripeptide has antioxidant properties and plays a key function in intracellular metabolite detoxification. The ability of glutathione to increase ARV drugs delivery to the brain via liposomes has been demonstrated [47].

\section{Biotechnological Advances in ARV Delivery}

In the previous sections we have presented the classical therapy approach of HIV infections / AIDS based on the use of ARV drugs. Although cART can reduce HIV replication and postpone the onset of HIV infection/AIDS, viral mutagenesis is common and can lead to ineffective ARV therapy. New prospects of HIV treatment include biotechnological approaches combining pharmacological compounds and, in particular, genetic therapy, which uses RNA interference (RNAi). RNAi mechanisms are used in the context of gene therapy to modulate/silence the expression of genes involved in disease. Small interfering RNA (siRNA) operate within the RNAi pathway and have become the focus of recent therapeutic applications. Double- and long-stranded RNAs interact with a complex of proteins in the cytoplasm of cells, which is then cut into small double-stranded RNAs (19-21 nucleotides), known as siRNA, via Dicer enzymes. When siRNA enters the RNA-induced silencing complex (RISC complex), its double strands are separated into two single strands (antisense and sense), the antisense strand (guide) binds to a messenger RNA (mRNA) with a complementary sequence, and the mRNA target is degraded by non-RISC-complex endonucleases, halting the production of the abnormally encoded protein or enzyme [148] (Figure 8).

The high potential of this strategy in comparison to others stems from the fact that when an appropriate siRNA is used, regular expression of any other gene implicated in other diseases is possible. The significance of studies developed by Fire and Mello [149] to discover RNAi cellular mechanisms was recognized with the Nobel Prize in Physiology or Medicine in 2006 [150]. 

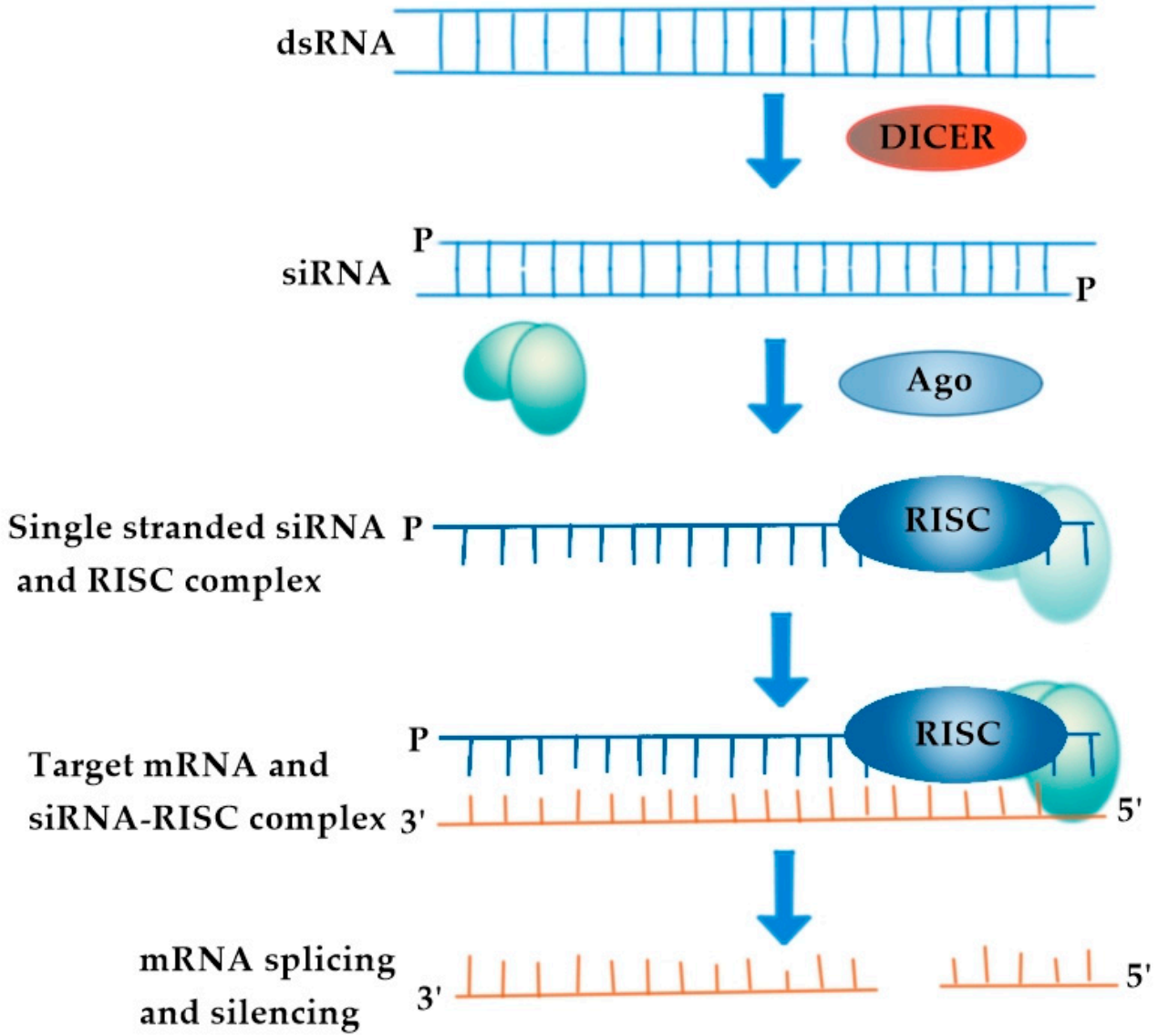

Figure 8. Mechanism of siRNA activity. RNA-induced silencing complex (RISC complex) is composed of Dicer and Argonaute protein (AGO).

Hence, siRNA-based therapeutics may offer a safer, effective, and longer-lasting approach that has demonstrated potential as a more personalized approach in the treatment of many diseases where enzyme activity is implicated, in which we may include HIV infections $[151,152]$. However, some obstacles must be solved before this therapeutic strategy can be used in clinical settings. These include improving delivery tactics and lowering costs. During the last decade, several research groups have worked on the topic of drug/nucleic acid co-delivery, mostly focusing on lipid-based nanocarriers. Indeed, the use of lipid-based nanocarriers, most commonly cationic charged liposomes or SLN, have several advantages, such as their ability to complex anionic nucleic acids and to protect RNAi from serum nucleases degradation and prolong blood circulation, which allows better distribution into the target tissues [148]. These systems are also essential for intracellular delivery, working as effective carriers for traversing the cytoplasmic membrane. For example, Kim et al. formulated a stabilized liposome for systemic administration of siRNA using a humanized mouse model to target lymphocyte function-associated antigen 1 (LFA-1), i.e., the predominant integrin found on all leukocytes. In vivo studies demonstrated a selective siRNA absorption by T cells and macrophages [153]. The opportunities for such continued innovation in formulating ARV drugs/nucleic acid co-delivery systems [148,153-155] will ensure continued research in this field, which should eventually lead to their clinical use.

ARV and siRNA co-delivery has been proposed as a promising biotechnological strategy for the treatment of Alzheimer's disease (AD) in a context other than HIV therapy. The amyloid hypothesis has emerged as the dominant theory to explain the molecular pathogenicity of $\mathrm{AD}$, following the identification of $\mathrm{A} \beta \mathrm{P}$ as the plaque-forming peptide aggregated and accumulated in the brain, and amyloid- $\beta$ precursor protein (APP) as the gene locus responsible for amyloid $\beta$-peptide $(A \beta P)$ production [156]. Accumulated plaques cause hyperphosphorylation of the microtubule-associated protein tau, which 
aggregates to form neurofibrillary tangles (NFT), synaptic dysfunction, cell death, and, eventually, AD [156]. Despite evidence supporting the amyloid hypothesis, many clinical trials focusing on $\mathrm{A} \beta$ components have failed to produce any AD-modifying therapies [156]. It has recently been discovered that SGR retro-inserts novel genomic complementary DNA into neuronal genomes and becomes dysregulated in $\mathrm{AD}$, producing numerous APP variant genes, transcripts, and $\mathrm{A} \beta \mathrm{P}$ that would remain in the brain in various potential forms (e.g., plaques, fibrils, prions, and soluble products) and may not be recognized by specific $A \beta$-antibodies used in the therapeutic attempts to target $A \beta P[156,157]$. As a result, SGR provides a novel mechanism for explaining $\mathrm{AD}$ pathogenesis and the failures of $\mathrm{A} \beta$-related clinical trials [156,157].

Human epidemiological data on 100,000 older HIV-infected patients ( $>=65$ years old) revealed that the world population's 10 percent prevalence of $\mathrm{AD}$ was not confirmed in these patients [156]. In fact, $1000 \mathrm{HIV}$-infected patients with AD were expected, but only one documented AD/HIV-infected case occurred [156]. This finding supports the notion that brain RT is involved in SGR, and its inhibition by ARV drugs or the silencing of its expression by siRNA is recently seen as a possible AD preventive and/or therapeutic intervention.

\section{Conclusions}

Notwithstanding the cART overall success, it continues to raise some serious concerns, and its effectiveness is hampered by some limitations such as ARV resistance mechanisms, prolonged treatment regimens, drug-drug interactions, toxicity effects, and pharmacokinetics issues. Therefore, innovative strategies such as lipid-based nanocarriers for ARV delivery appear to overcome physiological barriers. However, many issues must be addressed before we can reap the benefits of appropriate nanotechnology-based delivery systems that could improve ARV therapeutic outcomes. To begin with, most studies do not provide a thorough characterization of the lipid-based nanosystems developed or provide an incomplete or non-systematic formulation development methodology. Indeed, most studies do not consider the impact of nanosystems' composition on: (i) ARV encapsulation and loading efficiency; (ii) nanosystems' size and surface charge potential; (iii) nanosystems' ability to completely release the entrapped bioactives; and, finally, (iv) nanosystems' efficiency to deliver ARV to the virus reservoirs where their effects should be evaluated. Biotechnological applications of lipid nanocarriers loaded with anti-HIV therapeutics, such as the use of lipoplexes for siRNA delivery for AD, were also discussed. This promising bidirectional strategy helps ARV cross the BBB while halting SGR genome mutations that appear to be the cause of therapeutic AD failures.

Author Contributions: Conceptualization, M.L. and C.M.L.; writing-original draft preparation, M.J.F.; M.L., and C.M.L.; writing—review and editing, M.L. and C.M.L.; visualization, M.L.; supervision, M.L. and J.d.N.; funding acquisition, M.L. and C.M.L. All authors have read and agreed to the published version of the manuscript.

Funding: This research was funded by FCT/MCTES-Foundation for Science and Technology I.P. from the Minister of Science, Technology, and Higher Education (PIDDAC) and European Regional Development Fund (ERDF) by the COMPETE-Programa Operacional Factores de Competitividade (POFC) through the project CONCERT [POCI-01-0145-FEDER-032651 and PTDC/NANMAT/326512017] and the Strategic Funding UID/FIS/04650/2019 and "Contrato-Programa" UIDB/ 04469/2020 (CF-UM-UP and CBMA). Marlene Lúcio thanks FCT and ERDF for the doctoral position [CTTI-150/18-CF (1)] in the scope of the project CONCERT. Maria João Faria thanks FCT for PhD grant [2020.06561.BD].

Data Availability Statement: Data sharing not applicable.

Conflicts of Interest: The authors declare no conflict of interest. The funders had no role in the design of the study; in the collection, analyses, or interpretation of data; in the writing of the manuscript, or in the decision to publish the results. 


\section{List of Abbreviation}

\begin{tabular}{|c|c|}
\hline 3TC & lamivudine \\
\hline $\mathrm{A} \beta \mathrm{P}$ & amyloid $\beta$-peptide \\
\hline $\mathrm{ABC}$ & abacavir \\
\hline $\mathrm{AD}$ & Alzheimer's disease \\
\hline $\mathrm{AGO}$ & Argonaute protein \\
\hline AIDS & acquired immunodeficiency syndrome \\
\hline $\mathrm{APP}$ & amyloid- $\beta$ precursor proteinARV-antiretroviral \\
\hline ATV & atazanavir \\
\hline $\mathrm{Au}$ & gold \\
\hline AUC & area under the curve \\
\hline AV & aloe vera \\
\hline AZT & zidovudine \\
\hline AZT-M & zidovudine myristate \\
\hline AZTTP & azidothymidine $5^{\prime}$-triphosphate \\
\hline BBB & blood-brain barrier \\
\hline BHT & butylated hydroxy toluene \\
\hline Brij $^{\circledR}-35$ & polyoxyethylene (23) lauryl ether \\
\hline BSA & bovine serum albumin \\
\hline $\mathrm{CAB}$ & cabotegravir \\
\hline Capmul $^{\circledR} \mathrm{MCM}$ & mono/diglyceride of caprylic acid \\
\hline Capmul $^{\circledR}$ MCM EP & glycerol monocaprylocaprate \\
\hline Capmul $^{\circledR}$ PG 8 & propylene glycol monocaprylate \\
\hline Capryol $^{\mathrm{TM}} 90$ & propylene glycol monocaprylate \\
\hline Captex ${ }^{\circledR} \mathrm{P} 500$ & $\begin{array}{l}\text { triglycerides and esters prepared from fractionated vegetable oil sources } \\
\text { and fatty acids from coconuts and palm kernel oils }\end{array}$ \\
\hline $\mathrm{CC}_{50}$ & concentration at which $50 \%$ cells are viable \\
\hline cART & combined antiretroviral therapy \\
\hline CaSki & epidermoid cervical cancer cell line \\
\hline CCR5 & C-C chemokine receptor type 5 \\
\hline $\mathrm{CD} 4$ & cluster of differentiation 4 \\
\hline Chol & cholesterol \\
\hline $\mathrm{C}_{\max }$ & maximum concentration \\
\hline CNS & central nervous system \\
\hline Compritol $^{\circledR} 888$ ATO & glycerol dibehenate \\
\hline COVID-19 & coronavirus disease 2019 \\
\hline Cremophor $^{\circledR}$ EL & castor oil fatty acids, ethoxylated glycerol ester \\
\hline Cremophor ${ }^{\circledR}$ RH 40 & polyoxyl 40 hydrogenated castor oil \\
\hline CTAB & cetyltrimethylammonium bromide \\
\hline $\mathrm{d} 4 \mathrm{~T}$ & stavudine \\
\hline DCP & dicetyl phosphate \\
\hline $\mathrm{ddC}$ & zalcitabine \\
\hline ddI & didanosine \\
\hline ddI EC & enteric coated didanosine \\
\hline Dex-Prot & dextran-protamine; \\
\hline D.L. & drug loading \\
\hline DLV & delavirdine \\
\hline DMPC & 1,2-dimyristoyl-sn-glycero-3-phosphocholine \\
\hline DMPE & 1,2-dimyristoyl-sn-glycero-3-phosphoethanolamine \\
\hline DMPG & 1,2-dimyristoyl-sn-glycero-3-phospho-(1'-rac-glycerol) \\
\hline DNA & deoxyribonucleic acid \\
\hline DODAB & dioctadecyl dimethylammonium bromide \\
\hline DOPC & 1,2-dioleoyl-sn-glycero-3-phosphocholine \\
\hline DOPE & 1,2-dioleoyl-sn-glycero-3-phosphoethanolamine \\
\hline DOR & doravirine \\
\hline
\end{tabular}


DPPC

DPPE-PEG2000

DPPG

DPTAP

DRV

DSPC

DSPE

DSPG

DTG

Dynasan ${ }^{\circledR} 114$

Dynasan ${ }^{\circledR} 118$

EDPPC

E.E.

EFV

EPC

ETR

EVG

FIs

FPV

FTC

FTR

GC

Gelucire $^{\circledR}$ 44/14

Gelucire $^{\circledR} 50 / 13$

GI

GMO

GMS

gp41

gp120

GRAS

HAART

HBMECs

HEC-1-A

HG

HIV

HSA

HSPC

IBA

IDV

II

Labrasol $^{\circledR}$

Lauroglycol ${ }^{\mathrm{TM}}$

LDL

LFA-1

Lipoid $^{\circledR} \mathrm{S} 75$

$\log \mathrm{P}$

LP

LPV

$\mathrm{mAb}$

Maisine ${ }^{\circledR}$ 35-1

MAL

Man

MCC 1,2-dipalmitoyl-sn-glycero-3-phosphocholine

1,2-dipalmitoyl-sn-glycero-3-phosphoethanolamine-N-[methoxy-

(polyethylene

glycol)-2000]

1,2-dipalmitoyl-sn-glycero-3-phospho-(1'-rac-glycerol)

1,2-dipalmitoyl-3-trimethylammonium-propane (chloride salt)

darunavir

1,2-distearoyl-sn-glycero-3-phosphocholine

1,2-distearoyl-sn-glycero-3-phosphorylethanolamine

1,2-distearoyl-sn-glycero-3-phospho-(1'-rac-glycerol)

dolutegravir

trimyristin

glyceryl tristearate

cationic 1,2-dipalmitoylethyl-phosphatidylcholine

entrapment efficiency

efavirenz

egg phosphatidylcholine

etravirine

elvitegravir

fusion inhibitors

fosamprenavir

emtricitabine

fostemsavir tromethamine

glyceryl caprylate

lauroyl polyoxyl-32 glycerides

stearoyl polyoxyl-32 glycerides

gastrointestinal

glyceryl monooleate/monoolein

glyceryl monostearate

glycoprotein gp41

glycoprotein gp120

generally recognized as safe

highly effective antiretroviral therapy

human brain microvascular endothelial cells

human endometrial cancer-1

hydrogel

human immunodeficiency virus

human serum albumin

hydrogenated soy phosphatidylcholine

ibalizumab-uiyk

indinavir

integrase inhibitors

caprylocaproyl polyoxyl-8 glycerides

90 propylene glycol monolaurate

low-density lipoproteins

lymphocyte function-associated antigen 1

fat-free soybean phospholipids with 70\% PC

partition coefficient

liposome

lopinavir

83-14 monoclonal antibody

glyceryl monolinoleate

maleimide

mannose

microcrystalline cellulose 


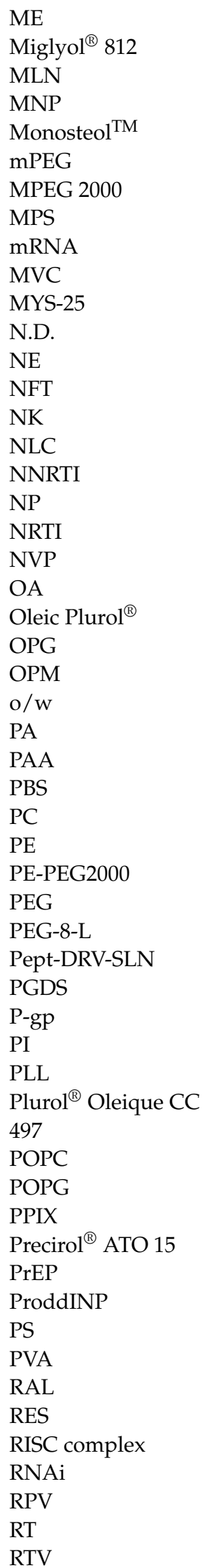

microemulsion

medium-chain triglycerides multiple lipid nanoparticles magnetic nanoparticles palmitate/stearate of propylene glycol methoxyl poly(ethylene glycol)

mono methoxy PEG 2000

mononuclear phagocyte system

messenger RNA

maraviroc

polyethylene glycol 25 stearate

no data

nanoemulsion

neurofibrillary tangles

natural killer

nanostructured lipid carriers

non-nucleoside reverse transcriptase inhibitors

nanoparticles

nucleoside reverse transcriptase inhibitors

nevirapine

oleic acid

polyglyceryl 6 dioleate

O-palmitoylgalactose

O-palmitoylmannose

oil-in-water

phenylalanine

poly(acrylic acid)

phosphate-buffered saline

phosphatidylcholine

phosphatidylethanolamine

dipalmitoylphosphatidylethanolamine-N-[poly(ethylene glycol)2000]

polyethylene glycol

octaoxyehtylene laurate ester

peptide grafted-darunavir loaded SLN

polyglyceryl-6-distearate

P-glycoprotein

protease inhibitors

poly(L-lysine hydrochloride

polyglyceryl-3 dioleate

1-palmitoyl-2-oleoyl-sn-glycero-3-phosphocholine

1-palmitoyl-2-oleoyl-sn-glycero-3-phospho-(1'-rac-glycerol)

protoporphyrin IX

glyceryl palmitostearate

pre-exposure prophylaxis

glycerolipidic prodrug of ddI

phosphatidylserine

poly vinyl alcohol

raltegravir

reticuloendothelial system

RNA-induced silencing complex

RNA interference

rilpivirine

reverse transcriptase

ritonavir 


\begin{tabular}{|c|c|}
\hline SA & stearylamine \\
\hline SARS-CoV-2 & severe acute respiratory syndrome coronavirus 2 \\
\hline SDS & sodium dodecyl sulfate \\
\hline SEDDS & self-emulsifying drug delivery systems \\
\hline SFV & sifuvirtide \\
\hline SGR & somatic gene recombination \\
\hline siRNA & small interference ribonucleic acid \\
\hline SL & soy lecithin \\
\hline SLN & solid lipid nanoparticles \\
\hline SM & sphingomyelin \\
\hline SMEDDS & self-micro emulsifying delivery systems \\
\hline Smix & surfactant and cosurfactant mix \\
\hline SNEDDS & self-nanoemulsifying drug delivery systems \\
\hline Softisan ${ }^{\circledR} 100$ & hydrogenated coco-glycerides \\
\hline Solutol ${ }^{\circledR}$ HS15 & polyoxyl 15 hydroxystearate \\
\hline SPC & soy phosphatidylcholine \\
\hline SQV & saquinavir \\
\hline SQVM & saquinavir mesylate \\
\hline S-SNEOF & solid self-nanoemulsifying oily formulations \\
\hline TAM & thymidine analog mutations \\
\hline $\mathrm{T}-20$ & enfuvirtide \\
\hline TDF & tenofovir disoproxil fumarate \\
\hline TFV & tenofovir \\
\hline TPV & tipranavir \\
\hline Transcutol ${ }^{\circledR} \mathrm{HP}$ & diethyleneglycol monoethyl ether \\
\hline Transcutol ${ }^{\circledR} \mathrm{P}$ & diethylene glycol monoethyl ether \\
\hline UNAIDS & Joint United Nations Program on HIV infection/AIDS \\
\hline U.S. FDA & United States Food and Drug Administration \\
\hline VE & $\alpha$-tocopherol \\
\hline $\mathrm{w} / \mathrm{o}$ & water-in-oil \\
\hline $\mathrm{w} / \mathrm{w}$ & weight/weight \\
\hline wt & weight \\
\hline
\end{tabular}

\section{References}

1. Boyapalle, S.; Mohapatra, S.; Mohapatra, S. Nanotechnology Applications to HIV Vaccines and Microbicides. J. Glob. Infect. Dis. 2012, 4, 62-68. [CrossRef] [PubMed]

2. das Neves, J.; Amiji, M.M.; Bahia, M.F.; Sarmento, B. Nanotechnology-based systems for the treatment and prevention of HIV/AIDS. Adv. Drug Deliv. Rev. 2010, 62, 458-477. [CrossRef]

3. UNAIDS. Global HIV\&AIDS Statistics-2019 Fact Sheet. Available online: https://www.unaids.org/en/resources/fact-sheet (accessed on 9 July 2021).

4. Nelson, A.G.; Zhang, X.; Ganapathi, U.; Szekely, Z.; Flexner, C.W.; Owen, A.; Sinko, P.J. Drug delivery strategies and systems for HIV / AIDS pre-exposure prophylaxis and treatment. J. Control. Release 2015, 219, 669-680. [CrossRef] [PubMed]

5. Gupta, U.; Jain, N.K. Non-polymeric nano-carriers in HIV/AIDS drug delivery and targeting. Adv. Drug Deliv. Rev. 2010, 62, 478-490. [CrossRef] [PubMed]

6. Simon, V.; Ho, D.D.; Abdool Karim, Q. HIV/AIDS epidemiology, pathogenesis, prevention, and treatment. Lancet 2006, 368, 489-504. [CrossRef]

7. Mallipeddi, R.; Rohan, L.C. Progress in antiretroviral drug delivery using nanotechnology. Int. J. Nanomed. $2010,5,533-547$.

8. Mamo, T.; Moseman, E.A.; Kolishetti, N.; Salvador-Morales, C.; Shi, J.; Kuritzkes, D.R.; Langer, R.; von Andrian, U.; Farokhzad, O.C. Emerging nanotechnology approaches for HIV/AIDS treatment and prevention. Nanomedicine 2010, 5, 269-285. [CrossRef] [PubMed]

9. Tatham, L.M.; Rannard, S.P.; Owen, A. Nanoformulation strategies for the enhanced oral bioavailability of antiretroviral therapeutics. Ther. Deliv. 2015, 6, 469-490. [CrossRef]

10. das Neves, J. Novel Approaches for the Delivery of Anti-HIV Drugs-What Is New? Pharmaceutics 2019, 11, 554. [CrossRef]

11. Lopes, C.M.; Silva, J.; Real Oliveira, M.E.C.D.; Lúcio, M. Lipid-based colloidal carriers for topical application of antiviral drugs. In Design of Nanostructures for Versatile Therapeutic Applications; Grumezescu, A.M., Ed.; William Andrew Publishing: Oxford, UK, 2018. [CrossRef]

12. Cavalcanti, S.M.T.; Nunes, C.; Costa Lima, S.A.; Soares-Sobrinho, J.L.; Reis, S. Optimization of nanostructured lipid carriers for Zidovudine delivery using a microwave-assisted production method. Eur. J. Pharm. Sci. 2018, 122, 22-30. [CrossRef] 
13. Garg, M.; Asthana, A.; Agashe, H.B.; Agrawal, G.P.; Jain, N.K. Stavudine-loaded mannosylated liposomes: In-vitro anti-HIV-I activity, tissue distribution and pharmacokinetics. J. Pharm. Pharmacol. 2006, 58, 605-616. [CrossRef] [PubMed]

14. Garg, M.; Dutta, T.; Jain, N.K. Reduced hepatic toxicity, enhanced cellular uptake and altered pharmacokinetics of stavudine loaded galactosylated liposomes. Eur. J. Pharm. Biopharm. 2007, 67, 76-85. [CrossRef] [PubMed]

15. Garg, M.; Jain, N.K. Reduced hematopoietic toxicity, enhanced cellular uptake and altered pharmacokinetics of azidothymidine loaded galactosylated liposomes. J. Drug Target. 2006, 14, 1-11. [CrossRef]

16. Gaur, P.K.; Mishra, S.; Bajpai, M.; Mishra, A. Enhanced oral bioavailability of efavirenz by solid lipid nanoparticles: In vitro drug release and pharmacokinetics studies. Biomed. Res. Int 2014, 2014, 363404. [CrossRef]

17. Harvie, P.; Désormeaux, A.; Bergeron, M.C.; Tremblay, M.; Beauchamp, D.; Poulin, L.; Bergeron, M.G. Comparative pharmacokinetics, distributions in tissue, and interactions with blood proteins of conventional and sterically stabilized liposomes containing 2', 3'-dideoxyinosine. Antimicrob. Agents Chemother. 1996, 40, 225-229. [CrossRef]

18. Heiati, H.; Tawashi, R.; Phillips, N.C. Solid lipid nanoparticles as drug carriers II. Plasma stability and biodistribution of solid lipid nanoparticles containing the lipophilic prodrug $3^{\prime}$-azido-3'-deoxythymidine palmitate in mice. Int. J. Pharm. 1998, 174, 71-80. [CrossRef]

19. Jain, S.; Tiwary, A.K.; Jain, N.K. Sustained and targeted delivery of an anti-HIV agent using elastic liposomal formulation: Mechanism of action. Curr. Drug Deliv. 2006, 3, 157-166. [CrossRef]

20. Nayak, D.; Boxi, A.; Ashe, S.; Thathapudi, N.C.; Nayak, B. Stavudine loaded gelatin liposomes for HIV therapy: Preparation, characterization and in vitro cytotoxic evaluation. Mater. Sci. Eng. C Mater. Biol. Appl. 2017, 73, 406-416. [CrossRef]

21. Perazzolo, S.; Shireman, L.M.; Koehn, J.; McConnachie, L.A.; Kraft, J.C.; Shen, D.D.; Ho, R.J.Y. Three HIV Drugs, Atazanavir, Ritonavir, and Tenofovir, Coformulated in Drug-Combination Nanoparticles Exhibit Long-Acting and Lymphocyte-Targeting Properties in Nonhuman Primates. J. Pharm. Sci. 2018, 107, 3153-3162. [CrossRef]

22. das Neves, J.; Nunes, R.; Rodrigues, F.; Sarmento, B. Nanomedicine in the development of anti-HIV microbicides. Adv. Drug Deliv. Rev. 2016, 103, 57-75. [CrossRef] [PubMed]

23. Sosnik, A.; Augustine, R. Challenges in oral drug delivery of antiretrovirals and the innovative strategies to overcome them. Adv. Drug Deliv. Rev. 2016, 103, 105-120. [CrossRef] [PubMed]

24. Amiji, M.M.; Vyas, T.K.; Shah, L.K. Role of nanotechnology in HIV / AIDS treatment: Potential to overcome the viral reservoir challenge. Discov. Med. 2006, 6, 157-162. [PubMed]

25. Fernandes, E.; Soares, T.B.; Goncalves, H.; Lucio, M. Spectroscopic Studies as a Toolbox for Biophysical and Chemical Characterization of Lipid-Based Nanotherapeutics. Front. Chem. 2018, 6, 323. [CrossRef] [PubMed]

26. Attama, A.A.; Mumuni, A.; Builders, P.F. Lipid Nanoparticulate Drug Delivery Systems: A Revolution in Dosage Form Design and Development. In Recent Advances in Novel Drug Carrier Systems; Sezer, A.D., Ed.; Intechopen: London, UK, 2012. [CrossRef]

27. Chopra, S.; Venkatesan, N.; Betageri, G.V. Liposomes as nanocarriers for anti-HIV therapy. Drug Deliv. Transl. Res. 2013, 3, 471-478. [CrossRef] [PubMed]

28. Diksha, M.; Anil, B.J. Lipid Based Nanocarriers for Delivery of Anti-HIV Drugs: A Mini Review. Nanosci. Nanotechnol. Asia 2018, 8, 172-185. [CrossRef]

29. Huda, A.; Prabha, S. Lipid Based Anti-Retroviral Nanocarriers: A Review of Current Literature and Ongoing Studies. Drug Deliv. Lett. 2017, 7, 71-82. [CrossRef]

30. Melhuish, A.; Lewthwaite, P. Natural history of HIV and AIDS. Medicine 2018, 46, 356-361. [CrossRef]

31. FDA. FDA-Approved HIV Medicines. Available online: https://hivinfo.nih.gov/understanding-hiv/fact-sheets/fda-approvedhiv-medicines (accessed on 9 July 2021).

32. Lisziewicz, J.; Tőke, E.R. Nanomedicine applications towards the cure of HIV. Nanomed. Nanotechnol. Biol. Med. 2013, 9, 28-38. [CrossRef]

33. Garg, A.B.; Nuttall, J.; Romano, J. The future of HIV microbicides: Challenges and opportunities. Antivir. Chem. Chemother. 2009, 19, 143-150. [CrossRef]

34. Nuttall, J. Microbicides in the prevention of HIV infection: Current status and future directions. Drugs 2010, 70, 1231-1243. [CrossRef]

35. Kumar, L.; Verma, S.; Prasad, D.N.; Bhardwaj, A.; Vaidya, B.; Jain, A.K. Nanotechnology: A magic bullet for HIV AIDS treatment. Artif. Cells Nanomed. Biotechnol. 2015, 43, 71-86. [CrossRef]

36. Zdanowicz, M.M. The pharmacology of HIV drug resistance. Am. J. Pharm. Educ. 2006, 70, 100. [CrossRef]

37. Clutter, D.S.; Jordan, M.R.; Bertagnolio, S.; Shafer, R.W. HIV-1 drug resistance and resistance testing. Infect. Genet. Evol. J. Mol. Epidemiol. Evol. Genet. Infect. Dis. 2016, 46, 292-307. [CrossRef]

38. Tang, M.W.; Shafer, R.W. HIV-1 Antiretroviral Resistance. Drugs 2012, 72, e1-e25. [CrossRef]

39. Blaise, P.; Clevenbergh, P.; Vaira, D.; Moutschen, M.; Dellamonica, P. HIV resistance to antiretroviral drugs: Mechanisms, genotypic and phenotypic resistance testing in clinical practice. Acta Clin. Belg. 2002, 57, 191-201. [CrossRef] [PubMed]

40. Margeridon-Thermet, S.; Shafer, R.W. Comparison of the Mechanisms of Drug Resistance among HIV, Hepatitis B, and Hepatitis C. Viruses 2010, 2, 2696-2739. [CrossRef] [PubMed]

41. Ammaranond, P.; Sanguansittianan, S. Mechanism of HIV antiretroviral drugs progress toward drug resistance. Fundam. Clin. Pharmacol. 2012, 26, 146-161. [CrossRef] 
42. Roy, U.; Rodríguez, J.; Barber, P.; das Neves, J.; Sarmento, B.; Nair, M. The potential of HIV-1 nanotherapeutics: From in vitro studies to clinical trials. Nanomedicine 2015, 10, 3597-3609. [CrossRef] [PubMed]

43. Siccardi, M.; Martin, P.; McDonald, T.O.; Liptrott, N.J.; Giardiello, M.; Rannard, S.; Owen, A. Nanomedicines for HIV therapy. Ther. Deliv. 2013, 4, 153-156. [CrossRef] [PubMed]

44. Margolis, A.M.; Heverling, H.; Pham, P.A.; Stolbach, A. A review of the toxicity of HIV medications. J. Med. Toxicol. Off. J. Am. Coll. Med. Toxicol. 2014, 10, 26-39. [CrossRef] [PubMed]

45. Chen, R.; Wang, T.; Song, J.; Pu, D.; He, D.; Li, J.; Yang, J.; Li, K.; Zhong, C.; Zhang, J. Antiviral Drug Delivery System for Enhanced Bioactivity, Better Metabolism and Pharmacokinetic Characteristics. Int. J. Nanomed. 2021, 16, 4959-4984. [CrossRef] [PubMed]

46. Osborne, O.; Peyravian, N.; Nair, M.; Daunert, S.; Toborek, M. The Paradox of HIV Blood-Brain Barrier Penetrance and Antiretroviral Drug Delivery Deficiencies. Trends Neurosci. 2020, 43, 695-708. [CrossRef] [PubMed]

47. Gomes, M.J.; Neves, J.d.; Sarmento, B. Nanoparticle-based drug delivery to improve the efficacy of antiretroviral therapy in the central nervous system. Int. J. Nanomed. 2014, 9, 1757-1769. [CrossRef]

48. Danta, C.C.; Piplani, P. Investigation of Molecular Properties of Antiretroviral Agents to Enhance CNS Penetration Abilities for the Treatment of Cognitive Impairment in HIV-Associated Neurocognitive Disorder. ACS Chem. Neurosci. 2020, 11, 2034-2038. [CrossRef] [PubMed]

49. Shao, J.; Kraft, J.C.; Li, B.; Yu, J.; Freeling, J.; Koehn, J.; Ho, R.J. Nanodrug formulations to enhance HIV drug exposure in lymphoid tissues and cells: Clinical significance and potential impact on treatment and eradication of HIV/AIDS. Nanomedicine 2016, 11, 545-564. [CrossRef]

50. Lúcio, M.; Lopes, C.M.; Fernandes, E.; Gonçalves, H.; Real Oliveira, M.E.C.D. Chapter 4. Organic Nanocarriers for Brain Drug Delivery. In Nanoparticles for Brain Drug Delivery; Vitorino, C., Jorge, A., Pais, A.A.C.C., Eds.; Jenny Stanford Publishing Pte. Ltd.: Singapore, 2021. [CrossRef]

51. Kalepu, S.; Manthina, M.; Padavala, V. Oral lipid-based drug delivery systems-An overview. Acta Pharm. Sin. B 2013, 3, 361-372. [CrossRef]

52. Faria, M.J.; Machado, R.; Ribeiro, A.; Goncalves, H.; Real Oliveira, M.; Viseu, T.; das Neves, J.; Lucio, M. Rational Development of Liposomal Hydrogels: A Strategy for Topical Vaginal Antiretroviral Drug Delivery in the Context of HIV Prevention. Pharmaceutics 2019, 11, 485. [CrossRef] [PubMed]

53. Jain, S.; Tiwary, A.K.; Jain, N.K. PEGylated elastic liposomal formulation for lymphatic targeting of zidovudine. Curr. Drug Deliv. 2008, 5, 275-281. [CrossRef] [PubMed]

54. Pokharkar, V.; Patil-Gadhe, A.; Palla, P. Efavirenz loaded nanostructured lipid carrier engineered for brain targeting through intranasal route: In-vivo pharmacokinetic and toxicity study. Biomed. Pharm. 2017, 94, 150-164. [CrossRef]

55. Vyas, A.; Jain, A.; Hurkat, P.; Jain, A.; Jain, S.K. Targeting of AIDS related encephalopathy using phenylalanine anchored lipidic nanocarrier. Colloids Surf. B Biointerfaces 2015, 131, 155-161. [CrossRef] [PubMed]

56. Soares, T.B.; Loureiro, L.; Carvalho, A.; Oliveira, M.; Dias, A.; Sarmento, B.; Lucio, M. Lipid nanocarriers loaded with natural compounds: Potential new therapies for age related neurodegenerative diseases? Prog. Neurobiol. 2018, 168, 21-41. [CrossRef]

57. Phillips, N.C.; Skamene, E.; Tsoukas, C. Liposomal encapsulation of 3'-azido-3'-deoxythymidine (AZT) results in decreased bone marrow toxicity and enhanced activity against murine AIDS-induced immunosuppression. J. Acquir. Immune Defic. Syndr. 1991, 4, 959-966.

58. Rao, K.S.; Ghorpade, A.; Labhasetwar, V. Targeting anti-HIV drugs to the CNS. Expert Opin. Drug Deliv. 2009, 6, 771-784. [CrossRef]

59. Sana, K.; Poorva, J.; Sourabh, J.; Richa, J.; Saurabh, B.; Aakanchha, J. Topical Delivery of Erythromycin Through Cubosomes for Acne. Pharm. Nanotechnol. 2018, 6, 38-47. [CrossRef]

60. Barriga, H.M.G.; Holme, M.N.; Stevens, M.M. Cubosomes: The Next Generation of Smart Lipid Nanoparticles? Angew. Chem. Int. Ed. 2019, 58, 2958-2978. [CrossRef]

61. Ahirrao, M.; Shrotriya, S. In vitro and in vivo evaluation of cubosomal in situ nasal gel containing resveratrol for brain targeting. Drug Dev. Ind. Pharm. 2017, 43, 1686-1693. [CrossRef]

62. Boge, L.; Bysell, H.; Ringstad, L.; Wennman, D.; Umerska, A.; Cassisa, V.; Eriksson, J.; Joly-Guillou, M.-L.; Edwards, K.; Andersson, M. Lipid-Based Liquid Crystals As Carriers for Antimicrobial Peptides: Phase Behavior and Antimicrobial Effect. Langmuir 2016, 32, 4217-4228. [CrossRef] [PubMed]

63. Chen, Y.; Angelova, A.; Angelov, B.; Drechsler, M.; Garamus, V.M.; Willumeit-Römer, R.; Zou, A. Sterically stabilized spongosomes for multidrug delivery of anticancer nanomedicines. J. Mater. Chem. B 2015, 3, 7734-7744. [CrossRef] [PubMed]

64. Kuo, Y.C.; Ko, H.F. Targeting delivery of saquinavir to the brain using 83-14 monoclonal antibody-grafted solid lipid nanoparticles. Biomaterials 2013, 34, 4818-4830. [CrossRef] [PubMed]

65. Chattopadhyay, N.; Zastre, J.; Wong, H.L.; Wu, X.Y.; Bendayan, R. Solid lipid nanoparticles enhance the delivery of the HIV protease inhibitor, atazanavir, by a human brain endothelial cell line. Pharm. Res. 2008, 25, 2262-2271. [CrossRef]

66. Garg, B.; Katare, O.P.; Beg, S.; Lohan, S.; Singh, B. Systematic development of solid self-nanoemulsifying oily formulations (S-SNEOFs) for enhancing the oral bioavailability and intestinal lymphatic uptake of lopinavir. Colloids Surf. B Biointerfaces 2016, 141, 611-622. [CrossRef]

67. Delshadi, R.; Bahrami, A.; McClements, D.J.; Moore, M.D.; Williams, L. Development of nanoparticle-delivery systems for antiviral agents: A review. J. Control. Release 2021, 331, 30-44. [CrossRef] 
68. Phillips, N.C.; Tsoukas, C. Liposomal encapsulation of azidothymidine results in decreased hematopoietic toxicity and enhanced activity against murine acquired immunodeficiency syndrome. Blood 1992, 79, 1137-1143. [CrossRef]

69. Désormeaux, A.; Harvie, P.; Perron, S.; Makabi-Panzu, B.; Beauchamp, D.; Tremblay, M.; Poulin, L.; Bergeron, M.G. Antiviral efficacy, intracellular uptake and pharmacokinetics of free and liposome-encapsulated $2^{\prime}, 3^{\prime}$-dideoxyinosine. AIDS 1994, 8 , 1545-1553. [CrossRef]

70. Ramana, L.N.; Sharma, S.; Sethuraman, S.; Ranga, U.; Krishnan, U.M. Stealth anti-CD4 conjugated immunoliposomes with dual antiretroviral drugs-Modern Trojan horses to combat HIV. Eur. J. Pharm. Biopharm. 2015, 89, 300-311. [CrossRef] [PubMed]

71. Aji Alex, M.R.; Chacko, A.J.; Jose, S.; Souto, E.B. Lopinavir loaded solid lipid nanoparticles (SLN) for intestinal lymphatic targeting. Eur. J. Pharm. Sci. 2011, 42, 11-18. [CrossRef]

72. Ansari, H.; Singh, P. Formulation and in-vivo Evaluation of Novel Topical Gel of Lopinavir for Targeting HIV. Curr. HIV Res. 2018, 16, 270-279. [CrossRef] [PubMed]

73. Bhalekar, M.; Upadhaya, P.; Madgulkar, A. Formulation and characterization of solid lipid nanoparticles for an anti-retroviral drug darunavir. Appl. Nanosci. 2017, 7, 47-57. [CrossRef]

74. Kuo, Y.C.; Chung, C.Y. Solid lipid nanoparticles comprising internal Compritol 888 ATO, tripalmitin and cacao butter for encapsulating and releasing stavudine, delavirdine and saquinavir. Colloids Surf. B Biointerfaces 2011, 88, 682-690. [CrossRef]

75. Makwana, V.; Jain, R.; Patel, K.; Nivsarkar, M.; Joshi, A. Solid lipid nanoparticles (SLN) of Efavirenz as lymph targeting drug delivery system: Elucidation of mechanism of uptake using chylomicron flow blocking approach. Int. J. Pharm. 2015, 495, 439-446. [CrossRef]

76. Vyas, S.P.; Subhedar, R.; Jain, S. Development and characterization of emulsomes for sustained and targeted delivery of an antiviral agent to liver. J. Pharm. Pharmacol. 2006, 58, 321-326. [CrossRef]

77. Bazzoli, C.; Jullien, V.; Tiec, C.L.; Rey, E.; Mentré, F.; Taburet, A.-M. Intracellular Pharmacokinetics of Antiretroviral Drugs in HIV-Infected Patients, and their Correlation with Drug Action. Clin. Pharmacokinet. 2010, 49, 17-45. [CrossRef] [PubMed]

78. Taylor, S.; Boffito, M.; Khoo, S.; Smit, E.; Back, D. Stopping antiretroviral therapy. AIDS 2007, 21, 1673-1682. [CrossRef] [PubMed]

79. Skanji, R.; Andrieux, K.; Lalanne, M.; Caron, J.; Bourgaux, C.; Degrouard, J.; Brisset, F.; Gueutin, C.; Chacun, H.; DereuddreBosquet, N.; et al. A new nanomedicine based on didanosine glycerolipidic prodrug enhances the long term accumulation of drug in a HIV sanctuary. Int. J. Pharm. 2011, 414, 285-297. [CrossRef]

80. Sudhakar, B.; Krishna, M.C.; Murthy, K.V.R. Factorial design studies of antiretroviral drug-loaded stealth liposomal injectable: PEGylation, lyophilization and pharmacokinetic studies. Appl. Nanosci. 2016, 6, 43-60. [CrossRef]

81. Jin, S.X.; Bi, D.Z.; Wang, J.; Wang, Y.Z.; Hu, H.G.; Deng, Y.H. Pharmacokinetics and tissue distribution of zidovudine in rats following intravenous administration of zidovudine myristate loaded liposomes. Pharmazie 2005, 60, 840-843. [PubMed]

82. Desai, J.; Thakkar, H. Darunavir-Loaded Lipid Nanoparticles for Targeting to HIV Reservoirs. AAPS Pharmscitech 2018, 19, 648-660. [CrossRef]

83. Garg, B.; Beg, S.; Kumar, R.; Katare, O.P.; Singh, B. Nanostructured lipidic carriers of lopinavir for effective management of HIV-associated neurocognitive disorder. J. Drug Deliv. Sci. Technol. 2019, 53. [CrossRef]

84. Gupta, S.; Kesarla, R.; Chotai, N.; Misra, A.; Omri, A. Systematic Approach for the Formulation and Optimization of Solid Lipid Nanoparticles of Efavirenz by High Pressure Homogenization Using Design of Experiments for Brain Targeting and Enhanced Bioavailability. Biomed Res. Int. 2017, 2017, 5984014. [CrossRef]

85. Jindal, A.B.; Bachhav, S.S.; Devarajan, P.V. In situ hybrid nano drug delivery system (IHN-DDS) of antiretroviral drug for simultaneous targeting to multiple viral reservoirs: An in vivo proof of concept. Int. J. Pharm. 2017, 521, 196-203. [CrossRef]

86. Khan, S.A.; Rehman, S.; Nabi, B.; Iqubal, A.; Nehal, N.; Fahmy, U.A.; Kotta, S.; Baboota, S.; Md, S.; Ali, J. Boosting the Brain Delivery of Atazanavir through Nanostructured Lipid Carrier-Based Approach for Mitigating NeuroAIDS. Pharmaceutics 2020, 12, 1059. [CrossRef] [PubMed]

87. Rojekar, S.; Fotooh Abadi, L.; Pai, R.; Mahajan, K.; Kulkarni, S.; Vavia, P.R. Multi-organ targeting of HIV-1 viral reservoirs with etravirine loaded nanostructured lipid carrier: An in-vivo proof of concept. Eur. J. Pharm. Sci. 2021, 164, 105916. [CrossRef] [PubMed]

88. Mahajan, H.S.; Mahajan, M.S.; Nerkar, P.P.; Agrawal, A. Nanoemulsion-based intranasal drug delivery system of saquinavir mesylate for brain targeting. Drug Deliv. 2014, 21, 148-154. [CrossRef] [PubMed]

89. Prabhakar, K.; Afzal, S.M.; Surender, G.; Kishan, V. Tween 80 containing lipid nanoemulsions for delivery of indinavir to brain. Acta Pharm. Sin. B 2013, 3, 345-353. [CrossRef]

90. Vyas, T.K.; Shahiwala, A.; Amiji, M.M. Improved oral bioavailability and brain transport of Saquinavir upon administration in novel nanoemulsion formulations. Int. J. Pharm. 2008, 347, 93-101. [CrossRef] [PubMed]

91. Kim, S.; Scheerer, S.; Geyer, M.A.; Howell, S.B. Direct cerebrospinal fluid delivery of an antiretroviral agent using multivesicular liposomes. J. Infect. Dis. 1990, 162, 750-752. [CrossRef] [PubMed]

92. Makabi-Panzu, B.; Lessard, C.; Beauchamp, D.; Desormeaux, A.; Poulin, L.; Tremblay, M.; Bergeron, M.G. Uptake and binding of liposomal 2',3'-dideoxycytidine by RAW 264.7 cells: A three-step process. J. Acquir. Immune Defic. Syndr. Hum. Retrovirol. 1995, 8 , 227-235. [CrossRef]

93. Katragadda, A.; Bridgman, R.; Betageri, G. Effect of liposome composition and cholesterol on the cellular uptake of stavudine by human monocyte/macrophages. Cell. Mol. Biol. Lett. 2000, 5, 483-494. 
94. Gagné, J.F.; Désormeaux, A.; Perron, S.; Tremblay, M.J.; Bergeron, M.G. Targeted delivery of indinavir to HIV-1 primary reservoirs with immunoliposomes. Biochim. Biophys. Acta 2002, 1558, 198-210. [CrossRef]

95. Kinman, L.; Brodie, S.J.; Tsai, C.C.; Bui, T.; Larsen, K.; Schmidt, A.; Anderson, D.; Morton, W.R.; Hu, S.L.; Ho, R.J. Lipid-drug association enhanced HIV-1 protease inhibitor indinavir localization in lymphoid tissues and viral load reduction: A proof of concept study in HIV-2287-infected macaques. J. Acquir. Immune Defic. Syndr. 2003, 34, 387-397. [CrossRef]

96. Garg, M.; Garg, B.R.; Jain, S.; Mishra, P.; Sharma, R.K.; Mishra, A.K.; Dutta, T.; Jain, N.K. Radiolabeling, pharmacoscintigraphic evaluation and antiretroviral efficacy of stavudine loaded $99 \mathrm{mTc}$ labeled galactosylated liposomes. Eur. J. Pharm. Sci. 2008, 33, 271-281. [CrossRef]

97. Lalanne, M.; Paci, A.; Andrieux, K.; Dereuddre-Bosquet, N.; Clayette, P.; Deroussent, A.; Re, M.; Vassal, G.; Couvreur, P.; Desmaele, D. Synthesis and biological evaluation of two glycerolipidic prodrugs of didanosine for direct lymphatic delivery against HIV. Bioorg. Med. Chem. Lett. 2007, 17, 2237-2240. [CrossRef]

98. Kapitza, S.B.; Michel, B.R.; van Hoogevest, P.; Leigh, M.L.; Imanidis, G. Absorption of poorly water soluble drugs subject to apical efflux using phospholipids as solubilizers in the Caco-2 cell model. Eur. J. Pharm. Biopharm. 2007, 66, 146-158. [CrossRef]

99. Kaur, C.D.; Nahar, M.; Jain, N.K. Lymphatic targeting of zidovudine using surface-engineered liposomes. J. Drug Target. 2008, 16, 798-805. [CrossRef]

100. Clayton, R.; Ohagen, A.; Nicol, F.; Del Vecchio, A.M.; Jonckers, T.H.; Goethals, O.; Van Loock, M.; Michiels, L.; Grigsby, J.; Xu, Z.; et al. Sustained and specific in vitro inhibition of HIV-1 replication by a protease inhibitor encapsulated in gp120-targeted liposomes. Antivir. Res. 2009, 84, 142-149. [CrossRef]

101. Ramana, L.N.; Sethuraman, S.; Ranga, U.; Krishnan, U.M. Development of a liposomal nanodelivery system for nevirapine. J. Biomed. Sci. 2010, 17, 57. [CrossRef]

102. Franquelim, H.G.; De-Sousa, F.F.; Veiga, A.S.; Santos, N.C.; Castanho, M.A.R.B. Cationic liposomes are possible drug-delivery systems for HIV fusion inhibitor sifuvirtide. Soft Matter 2011, 7, 11089-11092. [CrossRef]

103. Zidan, A.S.; Rahman, Z.; Khan, M.A. Product and process understanding of a novel pediatric anti-HIV tenofovir niosomes with a high-pressure homogenizer. Eur. J. Pharm. Sci. 2011, 44, 93-102. [CrossRef] [PubMed]

104. Ramana, L.N.; Sharma, S.; Sethuraman, S.; Ranga, U.; Krishnan, U.M. Investigation on the stability of saquinavir loaded liposomes: Implication on stealth, release characteristics and cytotoxicity. Int. J. Pharm. 2012, 431, 120-129. [CrossRef] [PubMed]

105. Xu, X.; Khan, M.A.; Burgess, D.J. A two-stage reverse dialysis in vitro dissolution testing method for passive targeted liposomes. Int. J. Pharm. 2012, 426, 211-218. [CrossRef]

106. Zidan, A.S.; Spinks, C.; Fortunak, J.; Habib, M.; Khan, M.A. Near-infrared investigations of novel anti-HIV tenofovir liposomes. AAPS J. 2010, 12, 202-214. [CrossRef]

107. Zidan, A.S.; Spinks, C.B.; Habib, M.J.; Khan, M.A. Formulation and transport properties of tenofovir loaded liposomes through Caco-2 cell model. J. Liposome Res. 2013, 23, 318-326. [CrossRef]

108. Spinks, C.B.; Zidan, A.S.; Khan, M.A.; Habib, M.J.; Faustino, P.J. Pharmaceutical characterization of novel tenofovir liposomal formulations for enhanced oral drug delivery: In vitro pharmaceutics and Caco-2 permeability investigations. Clin. Pharm. 2017, 9, 29-38. [CrossRef]

109. Patel, G.M.; Shelat, P.K.; Lalwani, A.N. QbD based development of proliposome of lopinavir for improved oral bioavailability. Eur. J. Pharm. Sci. 2017, 108, 50-61. [CrossRef]

110. Ahammed, V.; Narayan, R.; Paul, J.; Nayak, Y.; Roy, B.; Shavi, G.V.; Nayak, U.Y. Development and in vivo evaluation of functionalized ritonavir proliposomes for lymphatic targeting. Life Sci. 2017, 183, 11-20. [CrossRef]

111. Figueira, T.N.; Domingues, M.M.; Illien, F.; Cadima-Couto, I.; Todorovski, T.; Andreu, D.; Sagan, S.; Castanho, M.A.R.B.; Walrant, A.; Veiga, A.S. Enfuvirtide-Protoporphyrin IX Dual-Loaded Liposomes: In Vitro Evidence of Synergy against HIV-1 Entry into Cells. ACS Infect. Dis. 2020, 6, 224-236. [CrossRef]

112. Jain, S.; Tiwary, A.K.; Sapra, B.; Jain, N.K. Formulation and evaluation of ethosomes for transdermal delivery of lamivudine. AAPS Pharmscitech 2007, 8, E111. [CrossRef]

113. Chettupalli, A.K.; Ananthula, M.; Amarachinta, P.R.; Bakshi, V.; Yata, V.K. Design, Formulation, In-Vitro and Ex-Vivo Evaluation of Atazanavir Loaded Cubosomal Gel. Biointerface Res. Appl. Chem. 2021, 11, 12037-12054. [CrossRef]

114. Hosny, K.M. Nanosized Cubosomal Thermogelling Dispersion Loaded with Saquinavir Mesylate to Improve Its Bioavailability: Preparation, Optimization, in vitro and in vivo Evaluation. Int. J. Nanomed. 2020, 15, 5113-5129. [CrossRef]

115. Tomitaka, A.; Arami, H.; Huang, Z.; Raymond, A.; Rodriguez, E.; Cai, Y.; Febo, M.; Takemura, Y.; Nair, M. Hybrid magnetoplasmonic liposomes for multimodal image-guided and brain-targeted HIV treatment. Nanoscale 2017, 10, 184-194. [CrossRef]

116. Saiyed, Z.M.; Gandhi, N.H.; Nair, M.P.N. Magnetic nanoformulation of azidothymidine $5^{\prime}$-triphosphate for targeted delivery across the blood-brain barrier. Int. J. Nanomed. 2010, 5, 157-166. [CrossRef]

117. Nunes, R.; Bogas, S.; Faria, M.J.; Gonçalves, H.; Lúcio, M.; Viseu, T.; Sarmento, B.; das Neves, J. Electrospun fibers for vaginal administration of tenofovir disoproxil fumarate and emtricitabine in the context of topical pre-exposure prophylaxis. J. Control. Release 2021, 334, 453-462. [CrossRef]

118. Alukda, D.; Sturgis, T.; Youan, B.C. Formulation of tenofovir-loaded functionalized solid lipid nanoparticles intended for HIV prevention. J. Pharm. Sci. 2011, 100, 3345-3356. [CrossRef]

119. Shegokar, R.; Singh, K.K. Stavudine entrapped lipid nanoparticles for targeting lymphatic HIV reservoirs. Die Pharm. 2011, 66, 264-271. 
120. Kuo, Y.C.; Chung, J.F. Physicochemical properties of nevirapine-loaded solid lipid nanoparticles and nanostructured lipid carriers. Colloids Surf. B Biointerfaces 2011, 83, 299-306. [CrossRef]

121. Joshi, K.S.; Sharma, C.P.; Kalarikkal, N.; Sandeep, K.; Thomas, S.; Pothen, L.A. Evaluation of in-vitro cytotoxicity and cellular uptake efficiency of zidovudine-loaded solid lipid nanoparticles modified with Aloe Vera in glioma cells. Mater. Sci. Eng. C Mater. Biol. Appl. 2016, 66, 40-50. [CrossRef]

122. Javan, F.; Vatanara, A. Encapsulation of ritonavir in solid lipid nanoparticles: In-vitro anti-HIV-1 activity using lentiviral particles. J. Pharm. Pharmacol. 2017, 69, 1002-1009. [CrossRef]

123. Cavalcanti, S.M.T.; Nunes, C.; Lima, S.A.C.; Soares-Sobrinho, J.L.; Reis, S. Multiple Lipid Nanoparticles (MLN), a New Generation of Lipid Nanoparticles for Drug Delivery Systems: Lamivudine-MLN Experimental Design. Pharm. Res. 2017, 34, 1204-1216. [CrossRef]

124. Mahajan, K.Y.; Rojekar, S.V.; Desai, D.V.; Kulkarni, S.S.; Vavia, P.R. Efavirenz loaded nanostructured lipid carriers for efficient and prolonged viral inhibition in HIV-infected macrophages. Pharm. Sci. 2020. [CrossRef]

125. Shaikh, N.A.; Lala, R.R. Formulation development of dolutegravir sodium loaded nano lipid carriers for improved solubility and permeability. Int. J. Pharm. Sci. Res. 2021, 12, 3654-3665. [CrossRef]

126. Beloqui, A.; Solinís, M.; des Rieux, A.; Préat, V.; Rodríguez-Gascón, A. Dextran-protamine coated nanostructured lipid carriers as mucus-penetrating nanoparticles for lipophilic drugs. Int. J. Pharm. 2014, 468, 105-111. [CrossRef]

127. Dixit, G.R.; Mathur, V.B. Formulation and characterization of solid microemulsion of darunavir for enhanced solubility and dissolution. Int. J. Pharm. Sci. Res. 2015, 6, 3990-3999. [CrossRef]

128. Carvalho, A.L.; Silva, J.A.; Lira, A.A.; Conceição, T.M.; Nunes Rde, S.; de Albuquerque Junior, R.L.; Sarmento, V.H.; Leal, L.B.; de Santana, D.P. Evaluation of Microemulsion and Lamellar Liquid Crystalline Systems for Transdermal Zidovudine Delivery. J. Pharm. Sci. 2016, 105, 2188-2193. [CrossRef]

129. Kotta, S.; Khan, A.W.; Ansari, S.H.; Sharma, R.K.; Ali, J. Anti HIV nanoemulsion formulation: Optimization and in vitro-in vivo evaluation. Int. J. Pharm. 2014, 462, 129-134. [CrossRef]

130. McConville, C.; Friend, D. Development and characterisation of a self-microemulsifying drug delivery systems (SMEDDSs) for the vaginal administration of the antiretroviral UC-781. Eur. J. Pharm. Biopharm. 2013, 83, 322-329. [CrossRef]

131. Senapati, P.C.; Sahoo, S.K.; Sahu, A.N. Mixed surfactant based (SNEDDS) self-nanoemulsifying drug delivery system presenting efavirenz for enhancement of oral bioavailability. Biomed. Pharm. 2016, 80, 42-51. [CrossRef]

132. De Melo-Diogo, D.; Pais-Silva, C.; Dias, D.R.; Moreira, A.F.; Correia, I.J. Strategies to Improve Cancer Photothermal Therapy Mediated by Nanomaterials. Adv. Healthc. Mater. 2017, 6, 1700073. [CrossRef]

133. Chenthamara, D.; Subramaniam, S.; Ramakrishnan, S.G.; Krishnaswamy, S.; Essa, M.M.; Lin, F.-H.; Qoronfleh, M.W. Therapeutic efficacy of nanoparticles and routes of administration. Biomater. Res. 2019, 23, 20. [CrossRef]

134. Raoufi, E.; Bahramimeimandi, B.; Salehi-Shadkami, M.; Chaosri, P.; Mozafari, M.R. Methodical Design of Viral Vaccines Based on Avant-Garde Nanocarriers: A Multi-Domain Narrative Review. Biomedicines 2021, 9, 520. [CrossRef]

135. Danaei, M.; Kalantari, M.; Raji, M.; Samareh Fekri, H.; Saber, R.; Asnani, G.P.; Mortazavi, S.M.; Mozafari, M.R.; Rasti, B.; Taheriazam, A. Probing nanoliposomes using single particle analytical techniques: Effect of excipients, solvents, phase transition and zeta potential. Heliyon 2018, 4. [CrossRef]

136. Danaei, M.; Dehghankhold, M.; Ataei, S.; Hasanzadeh Davarani, F.; Javanmard, R.; Dokhani, A.; Khorasani, S.; Mozafari, M.R. Impact of Particle Size and Polydispersity Index on the Clinical Applications of Lipidic Nanocarrier Systems. Pharmaceutics 2018, 10, 57. [CrossRef] [PubMed]

137. Churchill, M.J.; Deeks, S.G.; Margolis, D.M.; Siliciano, R.F.; Swanstrom, R. HIV reservoirs: What, where and how to target them. Nat. Rev. Microbiol. 2016, 14, 55-60. [CrossRef]

138. Kerns, E.H.; Di, L. Chapter 5-Lipophilicity. In Drug-Like Properties: Concepts, Structure Design and Methods; Kerns, E.H., Di, L., Eds.; Academic Press: San Diego, CA, USA, 2008; pp. 43-47. [CrossRef]

139. Zhang, X.; Qi, J.; Lu, Y.; He, W.; Li, X.; Wu, W. Biotinylated liposomes as potential carriers for the oral delivery of insulin. Nanomed. Nanotechnol. Biol. Med. 2014, 10, 167-176. [CrossRef]

140. Benziger, D.P.; Edelson, J. Absorption from the vagina. Drug Metab. Rev. 1983, 14, 137-168. [CrossRef]

141. das Neves, J.; Palmeira-de-Oliveira, R.; Palmeira-de-Oliveira, A.; Rodrigues, F.; Sarmento, B. Vaginal Mucosa and Drug Delivery. In Mucoadhesive Materials and Drug Delivery Systems; Khutoryanskiy, V.V., Ed.; John Wiley \& Sons: Hoboken, NJ, USA, 2014; pp. 99-132. [CrossRef]

142. das Neves, J.; Amiji, M.; Sarmento, B. Mucoadhesive nanosystems for vaginal microbicide development: Friend or foe? Wires Nanomed. Nanobiotechnol. 2011, 3, 389-399. [CrossRef]

143. Parboosing, R.; Maguire, G.E.M.; Govender, P.; Kruger, H.G. Nanotechnology and the treatment of HIV infection. Viruses 2012, 4, 488-520. [CrossRef]

144. Pollock, S.; Dwek, R.A.; Burton, D.R.; Zitzmann, N. N-Butyldeoxynojirimycin is a broadly effective anti-HIV therapy significantly enhanced by targeted liposome delivery. AIDS 2008, 22, 1961-1969. [CrossRef]

145. Flasher, D.; Konopka, K.; Chamow, S.M.; Dazin, P.; Ashkenazi, A.; Pretzer, E.; Düzgünes, N. Liposome targeting to human immunodeficiency virus type 1-infected cells via recombinant soluble CD4 and CD4 immunoadhesin (CD4-IgG). Biochim. Biophys. Acta 1994, 1194, 185-196. [CrossRef] 
146. Bestman-Smith, J.; Gourde, P.; Désormeaux, A.; Tremblay, M.J.; Bergeron, M.G. Sterically stabilized liposomes bearing antiHLA-DR antibodies for targeting the primary cellular reservoirs of HIV-1. Biochim. Biophys. Acta Biomembr. 2000, 1468, 161-174. [CrossRef]

147. Kinman, L.; Bui, T.; Larsen, K.; Tsai, C.C.; Anderson, D.; Morton, W.R.; Hu, S.L.; Ho, R.J. Optimization of lipid-indinavir complexes for localization in lymphoid tissues of HIV-infected macaques. J. Acquir. Immune Defic. Syndr. 2006, 42, 155-161. [CrossRef] [PubMed]

148. Bobbin, M.L.; Burnett, J.C.; Rossi, J.J. RNA interference approaches for treatment of HIV-1 infection. Genome Med. 2015, 7, 50. [CrossRef]

149. Fire, A.; Xu, S.; Montgomery, M.K.; Kostas, S.A.; Driver, S.E.; Mello, C.C. Potent and specific genetic interference by doublestranded RNA in Caenorhabditis elegans. Nature 1998, 391, 806-811. [CrossRef]

150. Oliveira, A.C.N.; Fernandes, J.; Gonçalves, A.; Gomes, A.C.; Real Oliveira, M.E.C.D. Lipid-based Nanocarriers for siRNA Delivery: Challenges, Strategies and the Lessons Learned from the DODAX: MO Liposomal System. Curr. Drug Targets 2019, 20, 29-50. [CrossRef]

151. Berkhout, B.; ter Brake, O. Towards a durable RNAi gene therapy for HIV-AIDS. Expert Opin. Biol. Ther. 2009, 9, 161-170. [CrossRef]

152. Haasnoot, J.; Westerhout, E.M.; Berkhout, B. RNA interference against viruses: Strike and counterstrike. Nat. Biotechnol. 2007, 25, 1435-1443. [CrossRef]

153. Kim, S.-S.; Peer, D.; Kumar, P.; Subramanya, S.; Wu, H.; Asthana, D.; Habiro, K.; Yang, Y.-G.; Manjunath, N.; Shimaoka, M.; et al. RNAi-mediated CCR5 silencing by LFA-1-targeted nanoparticles prevents HIV infection in BLT mice. Mol. Ther. 2010, 18, 370-376. [CrossRef]

154. Karlsen, T.A.; Brinchmann, J.E. Liposome delivery of microRNA-145 to mesenchymal stem cells leads to immunological off-target effects mediated by RIG-I. Mol. Ther. 2013, 21, 1169-1181. [CrossRef]

155. Krebs, M.D.; Alsberg, E. Localized, targeted, and sustained siRNA delivery. Chem. Weinh. Bergstr. Ger. 2011, 17, 3054-3062. [CrossRef]

156. Kaeser, G.E.; Chun, J. Mosaic Somatic Gene Recombination as a Potentially Unifying Hypothesis for Alzheimer's Disease. Front. Genet. 2020, 11. [CrossRef]

157. Chai, G.; Gleeson, J.G. A newly discovered mechanism driving neuronal mutations in Alzheimer's disease. Nature 2018, 563, 631-632. [CrossRef] 